\title{
NIF Integrated Computer Controls System Description
}

P. VanArsdall

January 26, 1998

U.S. Department of Energy

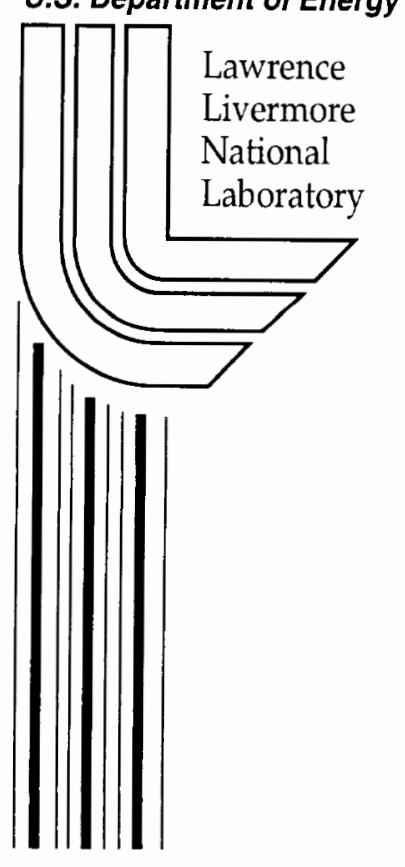





\section{DISCLAIMER}

This document was prepared as an account of work sponsored by an agency of the United States Government. Neither the United States Government nor the University of California nor any of their employees, makes any warranty, express or implied, or assumes any legal liability or responsibility for the accuracy, completeness, or usefulness of any information, apparatus, product, or process disclosed, or represents that its use would not infringe privately owned rights. Reference herein to any specific commercial product, process, or service by trade name, trademark, manufacturer, or otherwise, does not necessarily constitute or imply its endorsement, recommendation, or favoring by the United States Government or the University of California. The views and opinions of authors expressed herein do not necessarily state or reflect those of the United States Government or the University of California, and shall not be used for advertising or product endorsement purposes.

This work was performed under the auspices of the U. S. Department of Energy by the University of California, Lawrence Livermore National Laboratory under Contract No. W-7405-Eng-48.

This report has been reproduced directly from the best available copy.

Available electronically at http://www.doe.gov/bridge

Available for a processing fee to U.S. Department of Energy

and its contractors in paper from

U.S. Department of Energy

Office of Scientific and Technical Information

P.O. Box 62

Oak Ridge, TN 37831-0062

Telephone: (865) 576-8401

Facsimile: (865) 576-5728

E-mail: reports@adonis.osti.gov

Available for the sale to the public from

U.S. Department of Commerce

National Technical Information Service

5285 Port Royal Road

Springfield, VA 22161

Telephone: (800) 553-6847

Facsimile: (703) 605-6900

E-mail: orders@ntis.fedworld.gov

Online ordering: http://www.ntis.gov/ordering.htm

OR

Lawrence Livermore National Laboratory

Technical Information Department's Digital Library

http://www.llnl.gov/tid/Library.html 
Integrated Computer Controls System Description

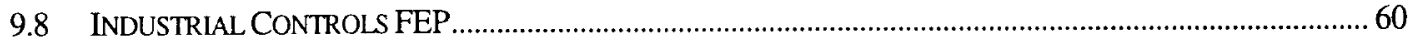

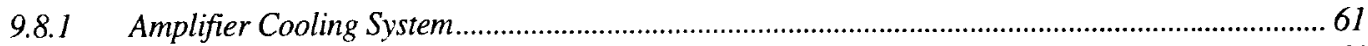

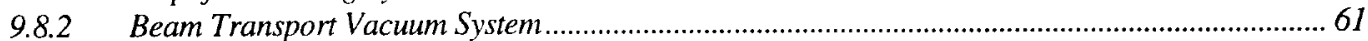

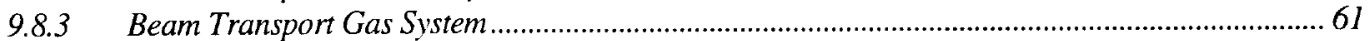

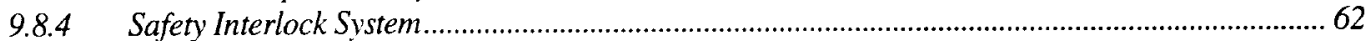

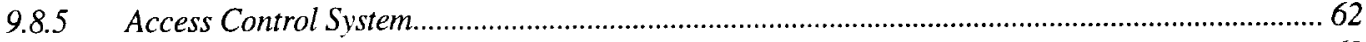

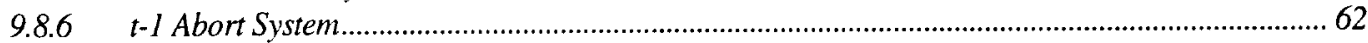

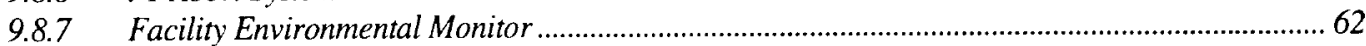

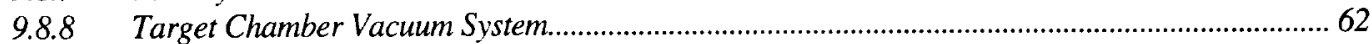

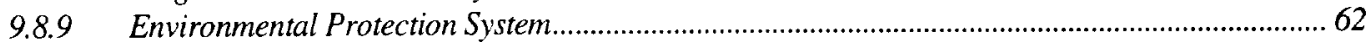

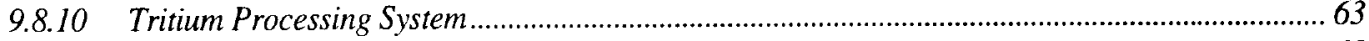

9.8.11 Personnel, Safety, and Occupational Access .............................................................................63 63

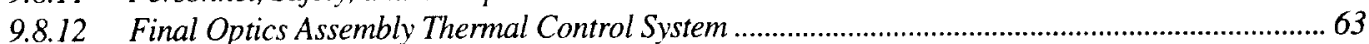

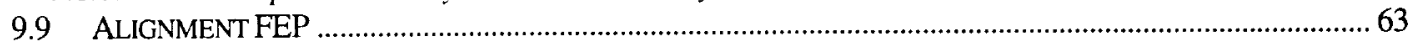

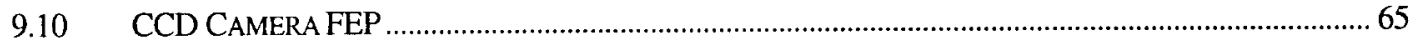

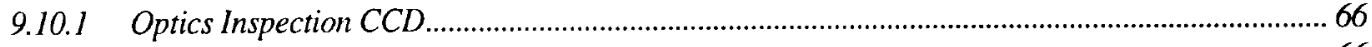

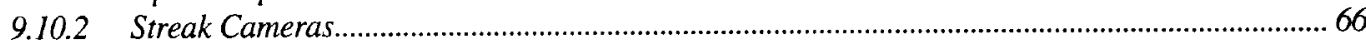

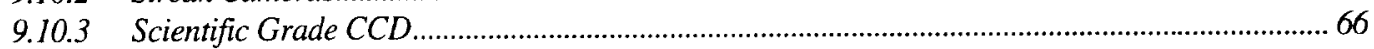

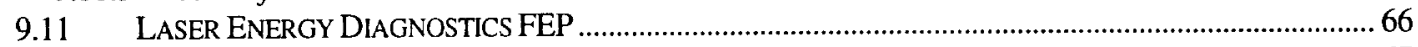

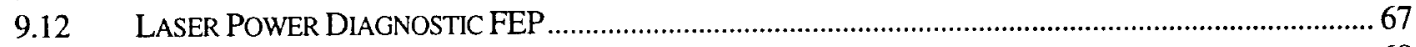

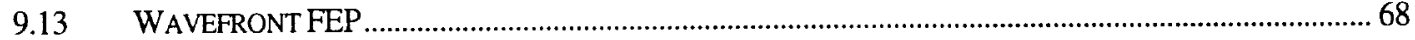

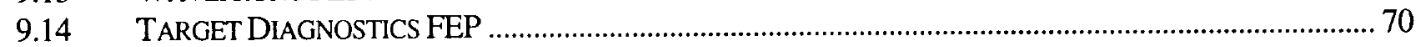

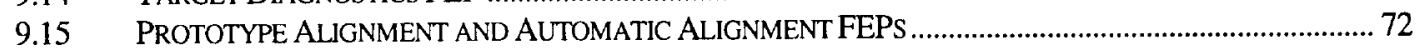

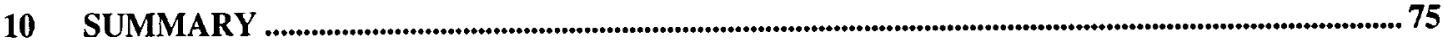

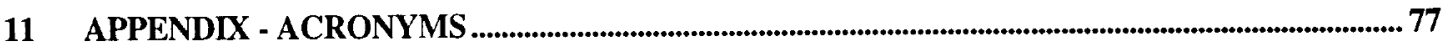

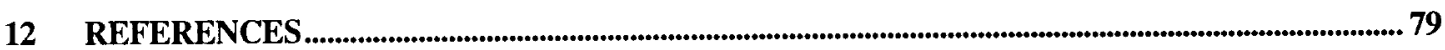


Integrated Computer Controls System Description

\section{Table of Figures}

FIGURE 1 NATIONAL IGNITION FACLLITY .

FIGURE 2 SIMPLIFIED NIF BEAM LINE SCHEMATIC

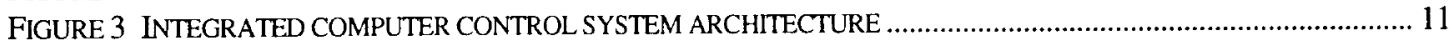

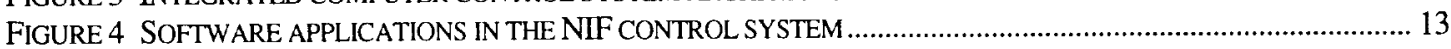

FIGURE 5 SHOT COUNTDOWN CLOCK GRAPHICAL USER INTERFACE .................................................................... 14

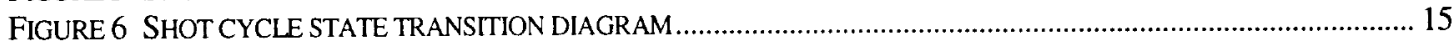

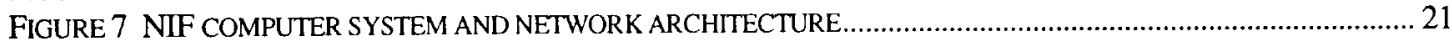

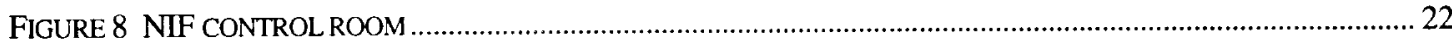

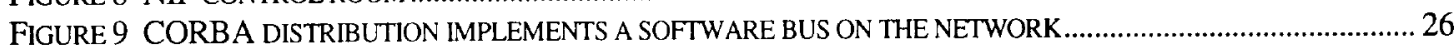

FIGURE 10 FLOWCHART OF THE ICCS SOFTWARE ENGINEERING PROCESS …………………………….................. 27

FIGURE 11 ICCS FRAMEWORK OBJECTS PLUG INTO THE CORBA SOFTWARE BUS ..................................................... 31

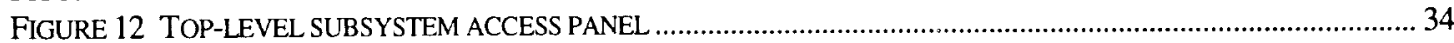

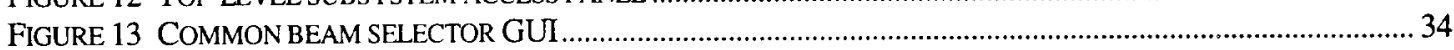

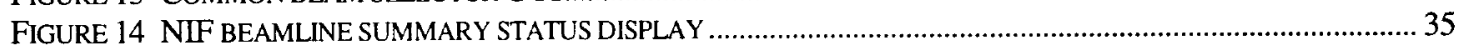

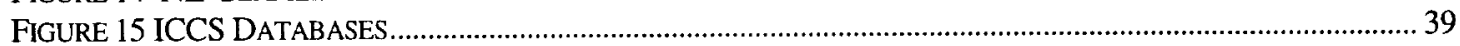

FIGURE 16 DATABASE INTERPROCESS COMMUNICATION …………................................................................ 40

FIGURE 17 OPTICAL PULSE GENERATOR FEP ARCHITECTURE …….................................................................... 44

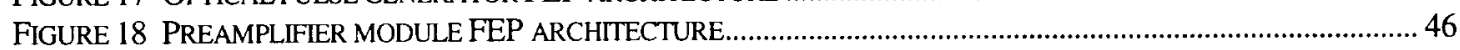

FIGURE 19 PEPC AND POLARIZER LOCATION IN THE NIF BEAMLINE …………………………………………...... 48

FigURE 20 PLASMA ELECTROdE POCKELS CELL SCHEMATIC............................................................................... 49

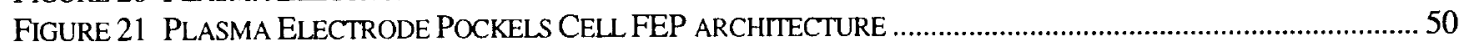

FIGURE 22 POWER CONDITIONING BANK MODULE SCHEMATIC ……………......................................................52

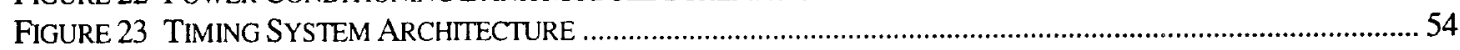

FIGURE 24 FACILITY TIMING DISTRIBUTION SUBSYSTEM DIAGRAM …………………………………………....5 55

FIGURE 25 AUTOMATIC ALIGNMENT FEP ARCHITECTURE........................................................................... 57

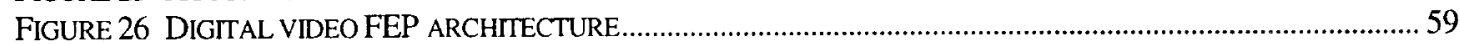

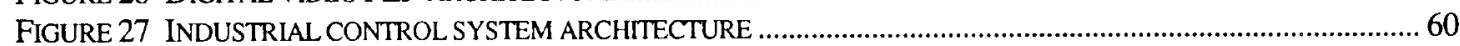

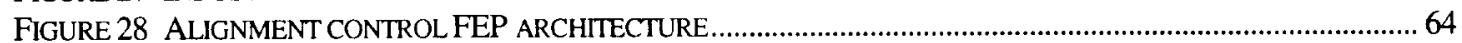

FIGURE 29 CCD CAMERA CONTROLLER FEP ARCHITECTURE ...........................................................................6 65

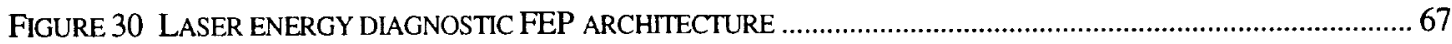

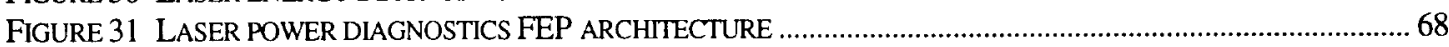

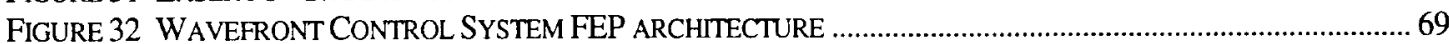

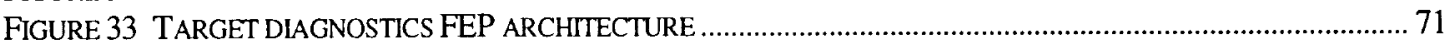

FIGURE 34 ALIGNMENT CONCEPTS LAB BEAMLINE CONFGGURATION …………………………........................... 72

FIGURE 35 ALIGNMENT PROTOTYPE FEP ARCHITECTURE ……………………………................................... 73

FIGURE 36 INTEGRATED COMPUTER CONTROL SYSTEM TESTBED …………………………................................... 75

\section{Tables}

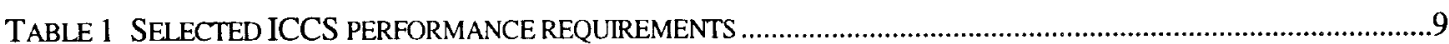

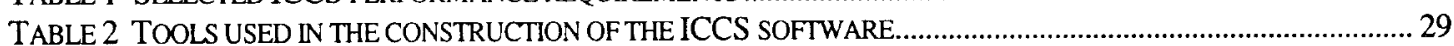

TABLE 3 SUMMARY OF FRONT-END PROCESSORS ..................................................................................... 43

TABLE 4 TIMING SYSTEM TRIGGER PERFORMANCE ..................................................................................... 53

This work was performed under the auspices of the U.S. Department of Energy by the University of California, Lawrence Livermore National Laboratory under Contract No. W-7405-Eng-48. 
Integrated Computer Controls System Description

This page intentionally left blank. 


\section{Introduction}

This System Description introduces the NIF Integrated Computer Control System (ICCS). The architecture is sufficiently abstract to allow the construction of many similar applications from a common framework. As discussed below, over twenty software applications derived from the framework comprise the NIF control system. This document lays the essential foundation for understanding the ICCS architecture.

The NIF design effort is motivated by the magnitude of the task. Figure 1 shows a cut-away rendition of the coliseum-sized facility. The NIF requires integration of about 40,000 atypical control points, must be highly automated and robust, and will operate continuously around the clock. The control system coordinates several experimental cycles concurrently, each at different stages of completion.

Furthermore, facilities such as the NIF represent major capital investments that will be operated, maintained, and upgraded for decades. The computers, control subsystems, and functionality must be relatively easy to extend or replace periodically with newer technology.

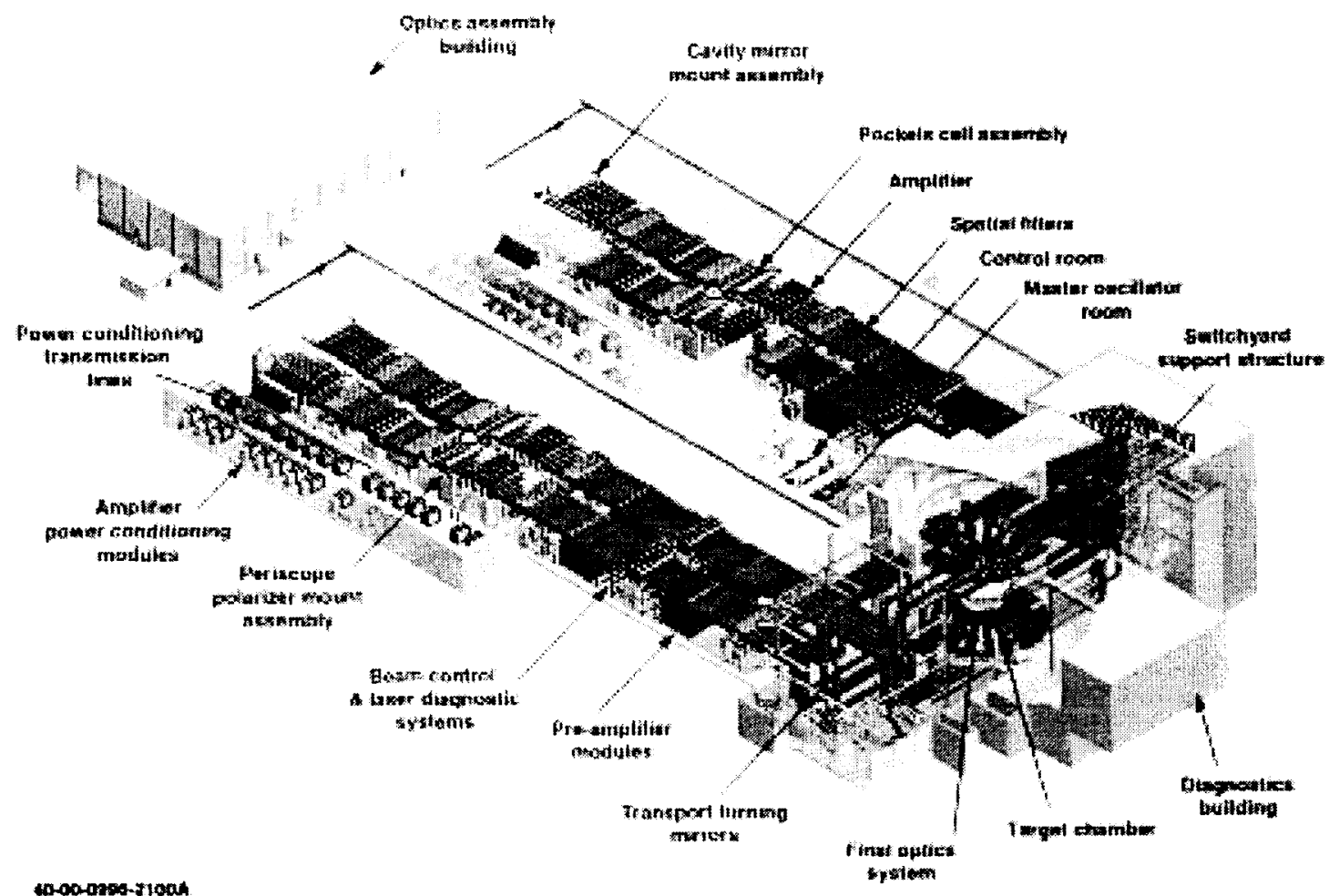

10-00-0ososisian

Figure 1 National Ignition Facility 
The NIF contains 192 laser beam lines that are focused on an ICF capsule at target chamber center [1]. The laser is organized into four clusters of 48 beams; two clusters are located in each of the two laser bays. Four capacitor banks pump flashlamps in the laser amplifiers, which store the energy until extracted by the laser pulse emitted by the master oscillator. The laser pulse is about $20 \mathrm{~ns}$ long. The system is capable of firing target shots every 8 hours, allowing time for the components to cool sufficiently to permit precise re-alignment of the laser beams onto the target.

In greatly simplified form, the beam line architecture is shown in Figure 2. A single Master Oscillator supplies complex laser pulses to 48 Preamplifier Modules that in turn are directed through the Preamplifier Beam Transport System and then injected into the Transport Spatial Filter. Once injected, the laser pulse travels (to the left in the figure) through the Two-pass Amplifier and into the Four-pass Amplifier section. Each pass through the amplifiers adds to the energy in the beam. The beam traverses the amplifier four times due to the action of the Plasma Electrode Pockels Cell, which switches the beam polarization during the second and third passes so that the beam is unaffected by the polarizer. On the fourth pass, the polarizer directs the beam back through the Two-pass amplifier for a second pass. The Spatial Filters remove unwanted high-frequency intensity spikes that would otherwise affect the beam. Harmonic converter crystals in the Final Optics Assembly convert the infrared laser light to ultraviolet and lenses focus the beam on the target. Many control points and computers are needed to control and diagnose the laser and target systems; these controls and their integrated operation are the subject of this document.

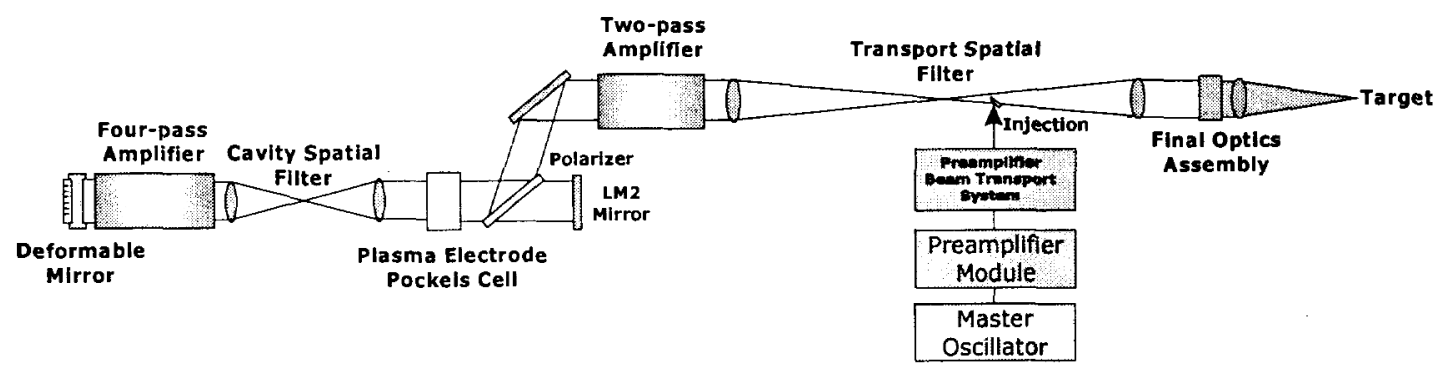

Figure 2 Simplified NIF beam line schematic

A brief summary of functional and performance requirements follows [Table 1].

Selected ICCS functional requirements:

- Provide graphical operator controls and equipment status

- Maintain records of system performance and operational history

- Automate predetermined control sequences (e.g. alignment)

- Coordinate shot setup, countdown, and shot data archiving

- Incorporate safety and equipment protection interlocks 
Integrated Computer Controls System Description

\begin{tabular}{|l|l|}
\hline Computer restart & $<30$ minutes \\
\hline Post-shot data recovery & $<5$ minutes \\
\hline Post-shot data archiving & $<30$ minutes \\
\hline Respond to broad-view status updates & $<10$ seconds \\
\hline Respond to alerts & $<1$ second \\
\hline Perform automatic alignment & $<1$ hour \\
\hline Transfer and display digital motion video & 10 frames per second \\
\hline Human-in-the-loop controls response & within $100 \mathrm{~ms}$ \\
\hline
\end{tabular}

Table 1 Selected ICCS performance requirements

Risks to the control system infrastructure are mitigated in the ICCS design by incorporating modularity, segmentation, and open-systems standards so that components and subsystems can be replaced at designated interface points if necessary. Risks to the control system software are managed through a modern object-oriented software framework that is used to construct all applications and that will be extensible and maintainable throughout the project life cycle. This framework is reusable across multiple applications (and indeed other projects) and offers interoperability among computers and operating systems by leveraging a common object request broker architecture (CORBA).

The Integrated Computer Control System (ICCS) architecture was created to address the general problem of providing distributed controls for medium to large-scale scientific (or other automated facilities) that do not require significant real-time capability within the supervisory software. The ICCS architecture is generally applicable to the class of event-driven control systems where client-server tactics are appropriate. This is the case for the NIF, as the shot time-line occurs over several hours and the shot can be suspended if necessary.

Some real-time control is inevitably necessary. Diagnostic instrumentation and controllers that are activated within two seconds of the laser shot are triggered by the Integrated Timing System. Other control loops requiring deterministic response are implemented at the edges of the integrated architecture in front-end computer equipment. Programmable logic controllers (PLC) in the Industrial Controls subsystem handle safety interlocks and utility controls for thermal, gas, and vacuum. In any event, no hard real-time control is implemented over the computer system network.

The following sections discuss several important views of the system:

- Section 2, Control System Architecture, presents a high-level description and simple model of the ICCS.

- Section 3, Supervisory Software Applications, discusses the eight supervisory control subsystems operated from the control room.

- Section 4, Computer System and Network, introduces the computer architecture and 700-node network topology. 
- Section 5, CORBA Distribution, explains the role of the object request broker in distributing software among the computers in the ICCS network.

- Section 6, Software Development Environment, discusses the tools and engineering process used to design and construct the object-oriented software

- Section 7, Reusable Supervisory Software Framework, provides an overview of the common components that are extended to construct all ICCS supervisory and front-end processor applications.

- Section 8, ICCS Databases, presents the five major database categories that provide persistence services for the ICCS operational data and NIF shot data.

- Section 9, Front End Processors, provides a summary of each of the front-end subsystems that interface to the NIF hardware.

- Section 10, Summary, discusses the current state of the ICCS development.

Acronyms are listed in the appendix and many of the terms used in this document are defined in Reference [2]. 


\section{Control System Architecture}

The ICCS is a layered architecture consisting of front-end processors (FEP) coordinated by a supervisory system [Figure 3]. Supervisory controls, which are hosted on UNIX workstations, provide centralized operator controls and status, data archiving, and integration services. FEP units are constructed from VME/VXI-bus or PCI-bus crates of embedded controllers and interfaces that attach to control points. FEP software provides the distributed services needed to operate the control points by the supervisory system. Functions requiring real-time implementation are allocated to software within the FEP or embedded controller, which is located at the edge of the architecture and therefore does not require communication over the local area network. The software is distributed among the computers and provides plugin software extensibility by using services from CORBA.

\section{Supervisory Controls}

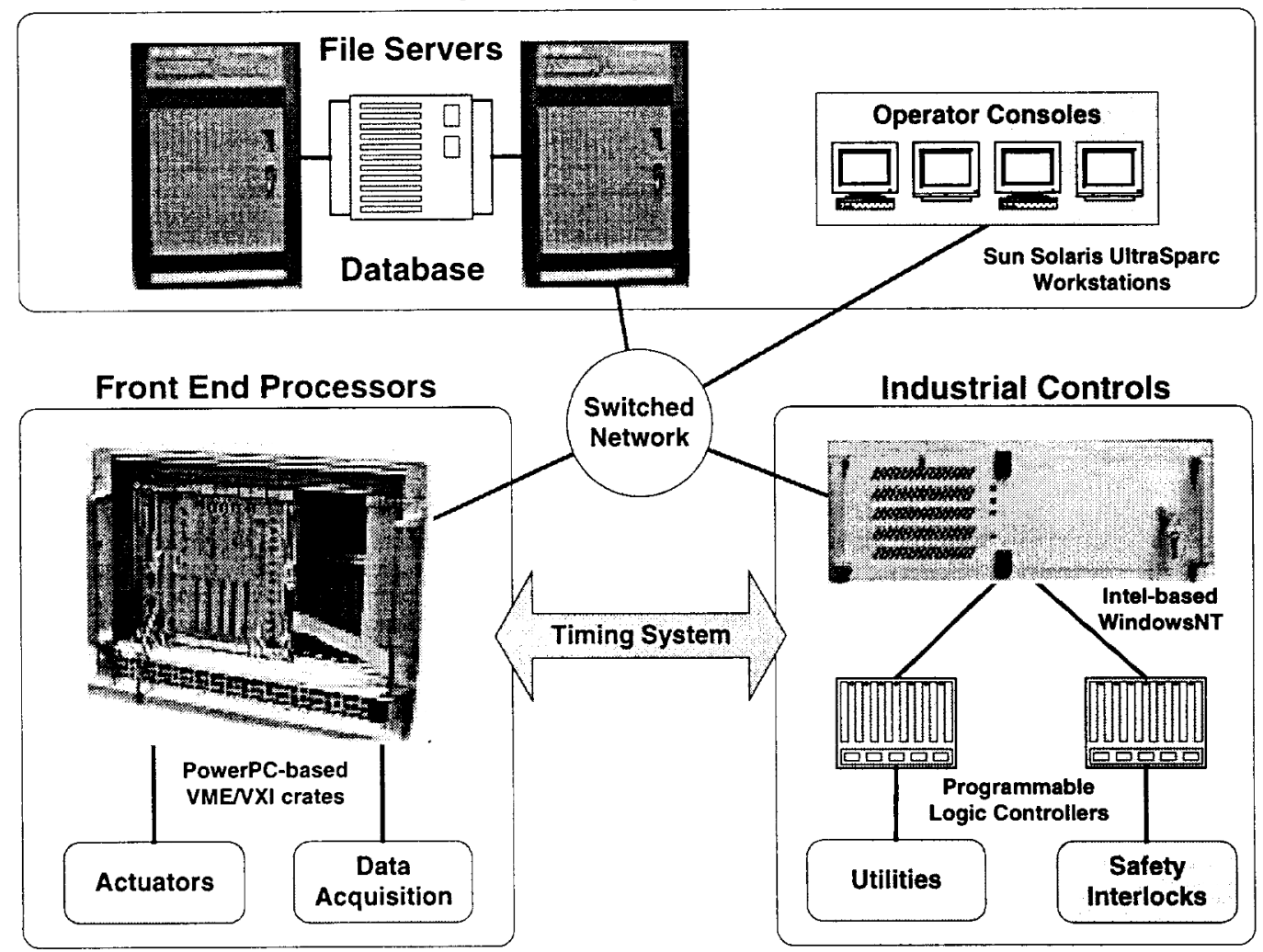

Figure 3 Integrated computer control system architecture

The operator console is a large software system that provides the human interface in the form of operator displays, data retrieval and processing, and coordination of control functions for the NIF. Supervisory software is partitioned into several cohesive subsystems, each of which controls a primary NIF subsystem such as alignment or power conditioning (see Supervisory Software Applications). Several 
databases are incorporated to manage both experimental data and data used during operations and maintenance. The subsystems are integrated to coordinate operation of laser and target area equipment. The supervisory software is responsible for duties ranging from configuration and control sequencing to data processing and archival.

Front-end processors implement the distributed portion of the ICCS by interfacing to the NIF control points (aka devices). The FEP software performs sequencing, data acquisition and reduction, and device control. The software framework includes a standard way for FEP units to be integrated into the supervisory system by providing a common distribution mechanism coupled with patterns for device configuration, control, and status monitoring functions.

NIF control points are sensors and actuators attached to interface boards plugged into the FEP backplane. In many cases, control points are handled by intelligent components that incorporate local microprocessors operated by small fixed programs (known as embedded controllers). In a few cases, remote devices attach to FEP units by utilizing a low-cost network of distributed microcontrollers known as a field bus. The software running in the embedded controller does much of the lowlevel work that would otherwise be imposed on the FEP. Example components in this layer are stepping motor controllers, photodiode detectors, and power supply controls.

The Industrial Controls subsystem contains a network of programmable logic controllers that reside below the FEP and attach to field devices implementing vacuum systems for the target chamber and spatial filters, argon gas controls for the beam tubes, and thermal gas conditioning for amplifier cooling. This system also implements the independent Safety Interlock system, which monitors doors, hatches, shutters, and other devices to establish and display the hazard levels in the facility due to high voltage, laser light, ionizing radiation, non-breathable atmosphere, and explosion/implosion. Permissive signals are issued to allow equipment to operate when conditions are safe. Unsafe conditions prevent operation and cause the affected systems to shut down. This system is capable of functioning autonomously to ensure safety without dependency on the rest of the control system. 


\section{Supervisory Software Applications}

The major functions of the ICCS supervisory software applications are:

- Conducting laser shots

- Providing status and control human interfaces for operators

- Processing and archiving laser and target performance data

- Coordinating manual device control

There are eight supervisory applications that perform these functions in collaboration with front-end processors as shown in Figure 4. Each of these is described below. Each description briefly describes the domain of the supervisor and then briefly describes its role in that domain, often with an emphasis on conducting a shot. Refer to section 9 for discussion on front-end processors.

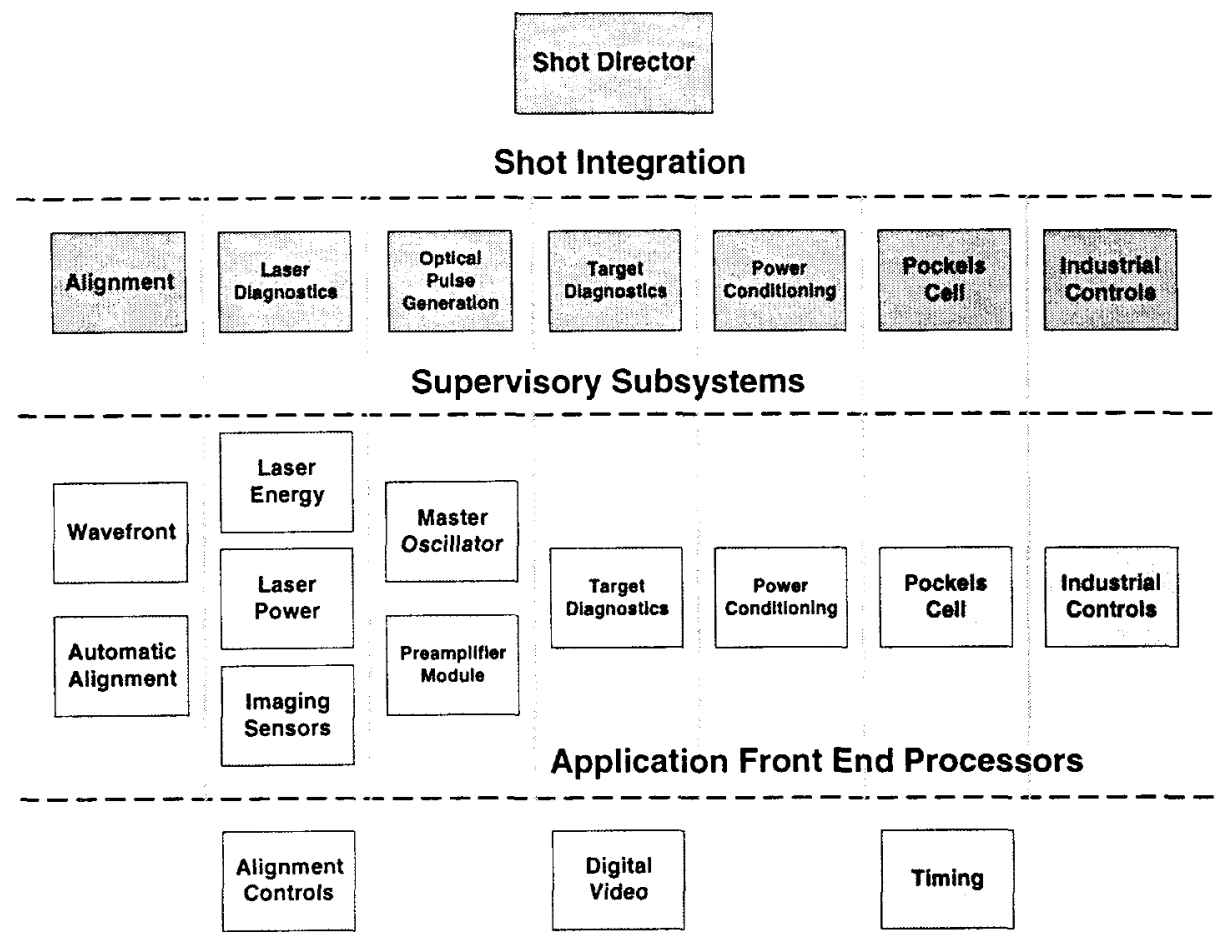

Service Front End Processors

Figure 4 Software applications in the NIF control system

\subsection{Shot Director}

The Shot Director software responsibilities are shot formulation and shot coordination. Shot formulation is carried out by the NIF Shot Scheduler ${ }^{1}$ using the

\footnotetext{
${ }^{1}$ NIF Shot Scheduler is the human being whose role is to create shots for the NIF from the experiments that have been approved by the NIF Shot Committee.
} 
ICCS Shot Scheduler ${ }^{2}$. Shot coordination is carried out by the NIF Shot Director ${ }^{3}$ using the ICCS Shot Director ${ }^{4}$.

NIF shots are comprised of one or more experiments. The types of NIF shots are detailed in NIF Shot Definitions, NIF-1863. The NIF Shot Scheduler uses the ICCS Shot Scheduler to formulate a shot from approved experiments and schedule it to be performed by the NIF operations staff. The NIF operations staff uses the ICCS supervisory subsystems, including the ICCS Shot Director, to perform the shot.

Once a NIF shot has been scheduled, the NIF Shot Director uses the ICCS Shot Director to select and coordinate its performance. The ICCS Shot Director coordinates the actions of the ICCS supervisory subsystems and interacts with the Timing FEP to perform a shot. Figure 5 shows the primary operator interface to the Shot Director application.

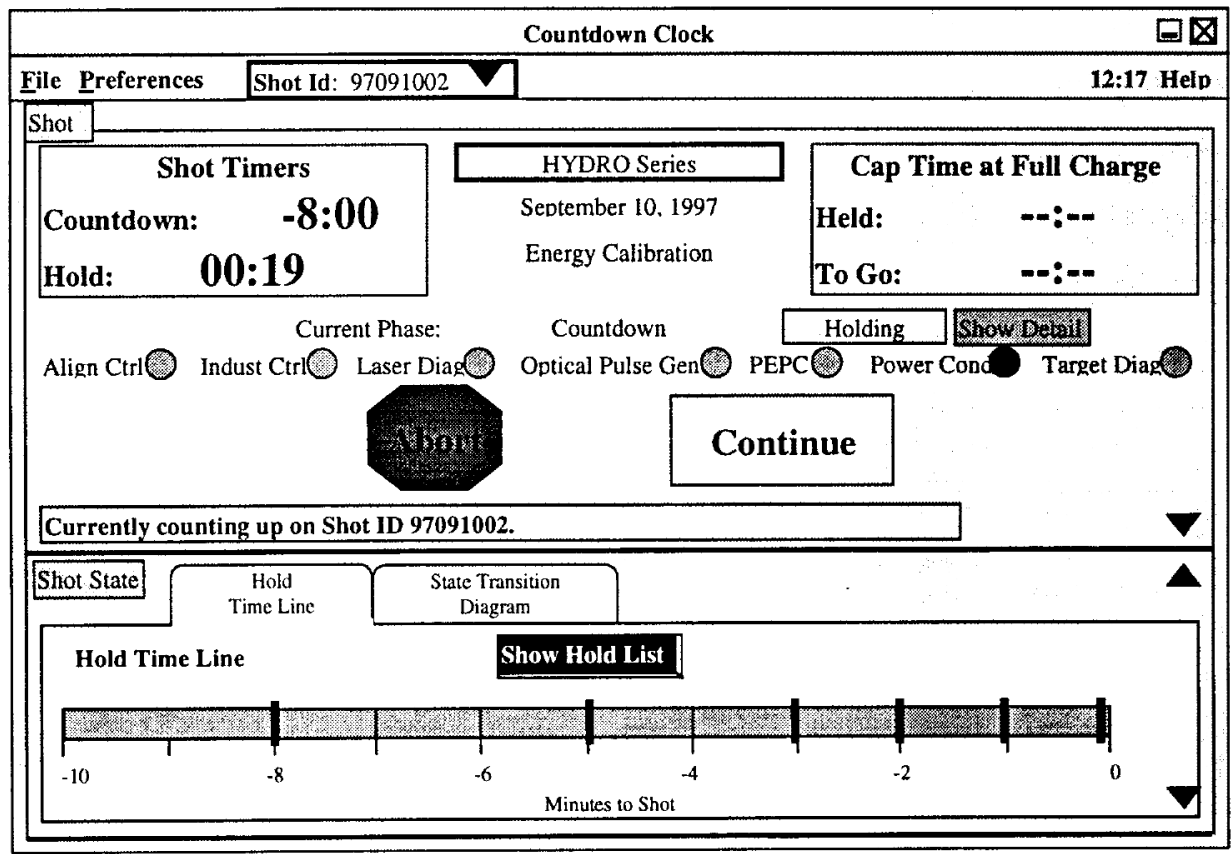

Figure 5 Shot countdown clock graphical user interface

Figure 6 describes the states of a NIF shot. The ICCS Shot Director advances the ICCS supervisory subsystems through these states to prepare for a shot and recover afterwards. For detailed information on the shot states and the activities that take place in each refer to [3].

\footnotetext{
${ }^{2}$ ICCS Shot Scheduler is the software that implements the Shot Scheduler functions.

${ }^{3}$ NIF Shot Director is the human being whose role is to oversee the operation of the NIF.

${ }^{4}$ ICCS Shot Director is the software that implements the Shot Director functions.
} 


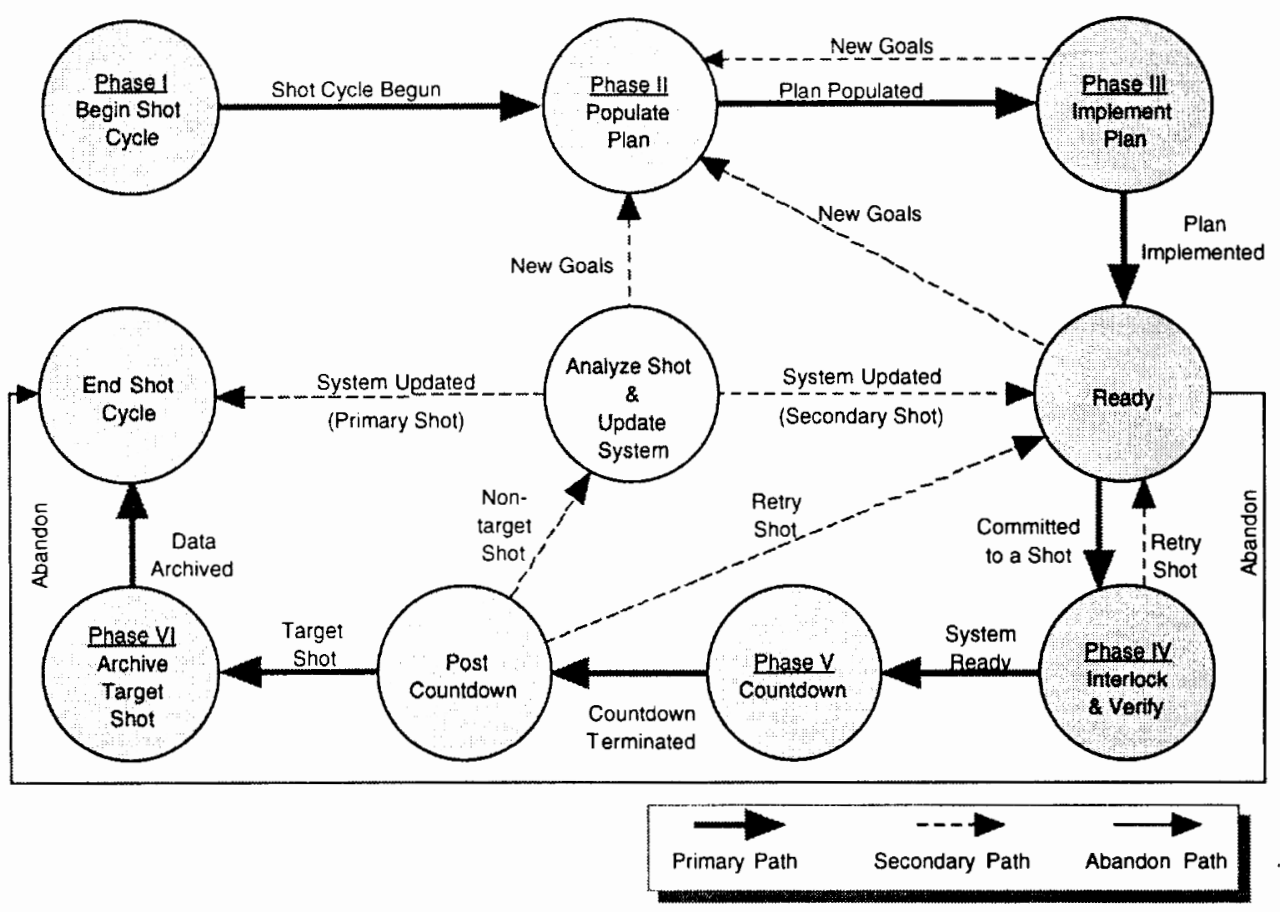

Figure 6 Shot cycle state transition diagram

\subsection{Supervisory Beam Control}

The Supervisory Beam Control software provides coordination and supervision of laser wavefront control and laser component alignment. Wavefront control is accomplished via a deformable mirror. Alignment is accomplished by pointing, centering, and rotating the beam through the laser components and pointing and focusing the laser beam on the target. Both automatic and manual alignment is possible. In order to perform these functions, the supervisory software coordinates the actions of three kinds of FEPs: Automatic Alignment, Wavefront Control, and Alignment Controls. In addition, the supervisory system provides graphical user interfaces for the FEPs, maintains beam control device parameters, displays beam control status, and provides a custom user-programmable scripting language to automate common beam control actions.

The activities of Automatic Alignment, Wavefront Control, and Laser Diagnostics are coupled via shared responsibilities and shared devices. The Beam Control Supervisor coordinates the shot preparation activities listed below in order to prevent conflicts.

The first beam control activity is the alignment of the laser preamplifier and the alignment laser used to align the main laser. At the start of main laser alignment, Wavefront Control performs a static wavefront correction based upon the time since the last shot. Automatic Alignment then roughly aligns the main laser, requests Wavefront Control to actively correct the wavefront, and completes the alignment. 
As beams are aligned, control of shared cameras is passed to Laser Diagnostics and Wavefront Control is asked to begin pointing maintenance in addition to actively controlling the wavefront. This latter activity continues until one second before the shot.

Should automatic alignment fail, beam control operators either correct or guide Automatic Alignment through the trouble spot and return the alignment operations to Automatic Alignment.

After the shot, the Beam Control Supervisor obtains the last alignment image from each component along all beams and stores them. It performs the same function for wavefront data except that shot data is also saved.

\subsection{Supervisory Industrial Controls}

The Industrial Controls (IC) Supervisor provides monitoring and control of environmental and safety parameters in the NIF. Parameters monitored by the Supervisor are concentrated in the Industrial Controls FEP computer(s) from the following Programmable Logic Controller (PLC) subsystems:

- Amplifier Cooling System

- Beam Transport Vacuum System

- Beam Transport Gas System

- Safety Interlock System

- Access control System

- T-1 Abort System

- Facility Environmental Monitor

- Target Chamber Vacuum System

- Environmental Protection System

- Tritium Processing System

- Final Optics Thermal Control

- Personnel Safety \& Access

Each of these PLC subsystems has its own extensive local monitoring and control capability.

During the NIF Shot Life Cycle the IC Supervisor monitors key environmental parameters to coordinate progressing through the Shot Cycle. The IC Supervisor will prevent the ICCS from moving to the next phase until environmental conditions are correct for supporting the functions that need to occur in the next phase.

Any condition that adversely affects a NIF shot will be signaled to the ICCS Shot Director and be displayed as Alerts on the ICCS Shot Director display. Some key activities during the NIF Shot Life Cycle are monitoring of Target Chamber and Laser Bay environmental conditions, Target and Laser Bay access permissive signals, and timely arming of the T-1 Abort System. After a countdown has completed, the 
Integrated Computer Controls System Description

IC Supervisor checks the status of the T-1 Abort System (see 9.8) to verify proper operation.

\subsection{Supervisory Laser Diagnostics}

The Laser Diagnostics subsystem provides functions for diagnosing performance of the NIF laser. This subsystem collects energy, transient, and image information from hardware sensors positioned strategically throughout each of the NIF beams. The primary purpose of the Laser Diagnostics subsystem is to summarize, display, and store laser performance data and ultimately diagnose and characterize the laser energy and power delivered to the target. In addition, the data is used to help diagnose performance anomalies and predict/detect potential damage to both active and passive optical components in each beam.

Beam diagnostic sensors in each NIF beam include devices in the Input Sensor Package, Back Reflection and Power Diagnostic Sensors, Output Sensor Package, and the 3w Output Calorimeter. Specialized sensor packages for calibration and precision diagnosis include the Roving 1w Calibration Calorimeter Assembly, Portable Streak Cameras, Precision 1w Diagnostic Stations, Precision 3w Diagnostic Stations, the Optics Inspection System, and the Pulse Synchronization Sensor.

Another important function of the Laser Diagnostics subsystem is calculating beam balance. The Beam Balance Control function is used to pre-configure laser gain and pulse shaping components for desired laser performance. Precision operation of NIF is achieved by adjusting these laser controls based on historical measurements combined with a model of the laser chain performance.

\subsection{Supervisory Optical Pulse Generation}

The NIF Optical Pulse Generation (OPG) System provides temporally and spatially formatted optical pulses with the correct energetics and optical characteristics required for each of the 192 NIF laser beams. It consists of three subsystems: the Master Oscillator (MOR), Preamplifier (PAM), and Preamplifier Beam Transport (PABTS).

The OPG System Supervisory Software is responsible for controlling and diagnosing the OPG Subsystem, as well as performing system functions such as configuration, data archival, history, and reporting. OPG Supervisory Software activities can be viewed from two perspectives: the Shot Director Supervisory Software, and an OPG Operator. The Shot Director Supervisory Software interface to the OPG Supervisory Software is primarily a software interface used for automatic sequencing of shotrelated activities. OPG Operator interaction with the OPG Supervisory Software occurs via Graphical User Interfaces. Operator interaction is required both for shot preparation and analysis, and maintenance activities.

The MOR subsystem must produce an initial light pulse meeting stringent temporal, optical, and energy characteristics. The Laser Diagnostics Supervisory Software is 
responsible for determining the desired pulse characteristics. The OPG Supervisory Software is mainly tasked with providing MOR pulse diagnostic data to Operators and Users.

The PAM subsystem amplifies, smooths, and shapes the initial optical pulse. The OPG Supervisory Software does PAM equipment set up and verification for shots, and provides for maintenance activities by Operators and Technicians.

The PABTS subsystem directs the output of the PAM into the Transport Spatial Filter Injection Port. The OPG Supervisory Software controls and monitors PABTS ion pumps.

\subsection{Supervisory Power Conditioning}

The Power Conditioning Supervisory software, or PCS, is responsible for high-level control and management of firing the main laser amplifiers. Thousands of capacitors along with their circuitry are grouped into modules that are used to feed thousands of flashlamps in the main laser amplifiers. Information about the controllable devices contained in these modules must be maintained. In addition, the ability to manually control the devices for testing purposes must be provided. It is up to the PCS to support these as well as the amplification operations.

The PCS performs its functions by coordinating the activities of one or more Power Conditioning Front-End Processors. PCS also provides graphical user interfaces for the power conditioning operators.

To perform a shot, the PCS provides voltage settings for each participating capacitor bank which Laser Diagnostics uses to calculate Optical Pulse Generation (OPG) output parameters. Once the shot parameters have been established and the hardware reserved, the countdown begins. After other ICCS components have completed certain operations, the PCS is allowed to begin charging the capacitor banks, the status of which it monitors and displays to the NIF Operator. The PCS also maintains timers that track how long a given bank has been at full charge. Should an anomaly be detected, by the FEPs or the NIF Operator, or a bank found to be holding full charge beyond damage threshold the PCS will submit a request to the Shot Director that the shot be aborted. Otherwise, if the shot proceeds, the PCS will collect circuit data from the FEP in part to determine if any anomalies occurred during the shot. If none were found, the PCS signals Industrial Controls that it can proceed with cooling the flashlamp cavities. The PCS then archives shot and performance data.

Between shots, operators can use the PCS to perform a pre-ionization procedure to check the amplifier flagships. They may also use it to maintain device parameters, generate reports on archive or performance data, or manually control power conditioning devices. 
Integrated Computer Controls System Description

\subsection{Supervisory Plasma Electrode Pockels Cell}

The Plasma Electrode Pockel's Cell (PEPC) serves as an optical switch facilitating multi-pass amplification within the main laser amplifiers. It does this by coordinating the activities of the PEPC FEPs. PEPC Supervisory software provides control, monitoring and coordinated operation of the PEPC. Manual control provides diagnostic and maintenance capabilities of individual PEPC device components. PEPC devices are typically operated in a coordinated scheme with other Integrated Computer Control System software components and the integrated timing system to conduct a NIF shot.

The PEPC is normally inactive for much of the NIF shot life cycle. During shot setup the PEPC Supervisor verifies that PEPC can meet the operational requirements of the shot. To accomplish this, the PEPC Supervisor verifies that the required PEPCs are available for the beams participating in the shot. The Supervisor signals that PEPC devices are functioning correctly before allowing the shot to proceed to the phase where amplifier capacitor charging begins.

At a predetermined time during the countdown, the PEPC Supervisor instructs the PEPC devices to begin their preparation and firing sequence. When the PEPC FEP has verified that the PEPC devices are firing correctly a message is sent to the ICCS Shot Director allowing capacitor charging to begin. The PEPC Supervisor continues monitoring PEPC operation and alerts the ICCS Shot Director of any anomalies that may occur. After completion of a shot, the Supervisor instructs the PEPC to stop firing and to perform their shutdown sequence. The Supervisor is also responsible for coordinating PEPC data archive activity.

\subsection{Supervisory Target Diagnostics}

The Supervisory Target Diagnostics software coordinates and supervises the collection of data resulting from a laser pulse interacting with a target. In addition, the Supervisory Target Diagnostics system also provides general status, all graphical user interfaces, quick-look data processing, and archival functions. The Supervisory Target Diagnostics system functions in both an unclassified and classified environment.

The target diagnostics system consists of Diagnostic Controllers, Target Diagnostic FEPs, and the Supervisory Target Diagnostics system. The Diagnostic Controllers (stand alone systems that conform to a set of NIF diagnostic interface standards for both hardware and software) directly control the diagnostics. The Target Diagnostic FEP acts as a protocol translator for the Diagnostic Controllers and the supervisory system. Data about diagnostics are dynamically learned by the Target Diagnostic Supervisor via the services of the Target Diagnostic FEP. This arrangement allows target diagnostics to be dynamically changed without requiring changes to the Supervisor or the Target Diagnostic FEP. 
Before a shot, diagnostics are selected for participation in the shot, the data produced are identified, storage space is reserved, Quick-look processing is specified, and retrieval priorities are set. All status and limited control of target diagnostics are performed via graphical user interfaces consisting of three panels: General status, Specific status and control, and Processing. If the Diagnostic Controller has a specific graphical user interface, an operator may activate it on the NIF console.

After a shot, the data is automatically archived. A priority may be associated with each data element so that data requiring early Quick-look processing will be obtained first. Quick-look processing (optional) may be performed in order to confirm the validity of the data. Data-processing command scripts (provided by each diagnostic) specify quick-look processing. Automatic and manual activation of the processing is possible. 
Integrated Computer Controls System Description

\section{Computer System and Network}

Figure 7 shows the NIF computer system, which is comprised several hundred processors distributed over an area roughly two football fields in size. The main control room contains seven graphics consoles, each of which houses two workstations with dual displays. The applications discussed above are normally assigned to execute and be operated from one primary console, although the software can easily be moved and is intended to be operated from remote graphics terminals located near the front end equipment. A pair of file servers provides disk storage and archival databases for the entire system as well as well as hosting centralized management and naming services necessary for coordinating the facility operation. The ICCS will operate, at reduced efficiency, with only one of the file servers in the event of a failure in the other.

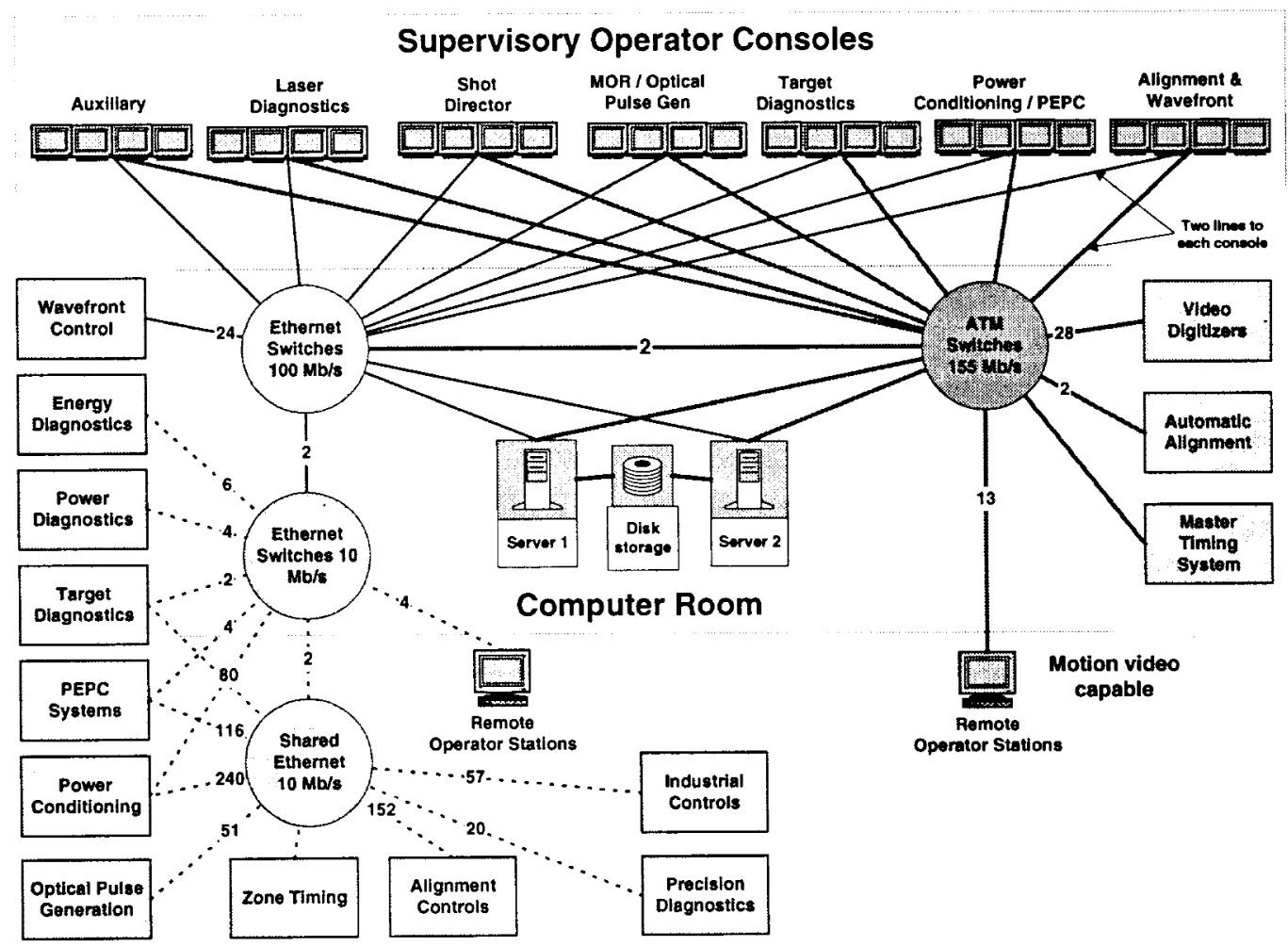

Figure 7 NIF computer system and network architecture

The operator console workstations communicate with distributed front-end processors that implement the localized control and monitoring functions. Different types of FEPs for the various subsystems are located in 19" equipment racks throughout the facility. There are about 700 network-attached devices within the facility. Since many of the FEPs are diskless 
systems, the network is designed to support the simultaneous bootstrapping of all FEPs. ICCS requirements specify that all systems will be restarted within 30 minutes.

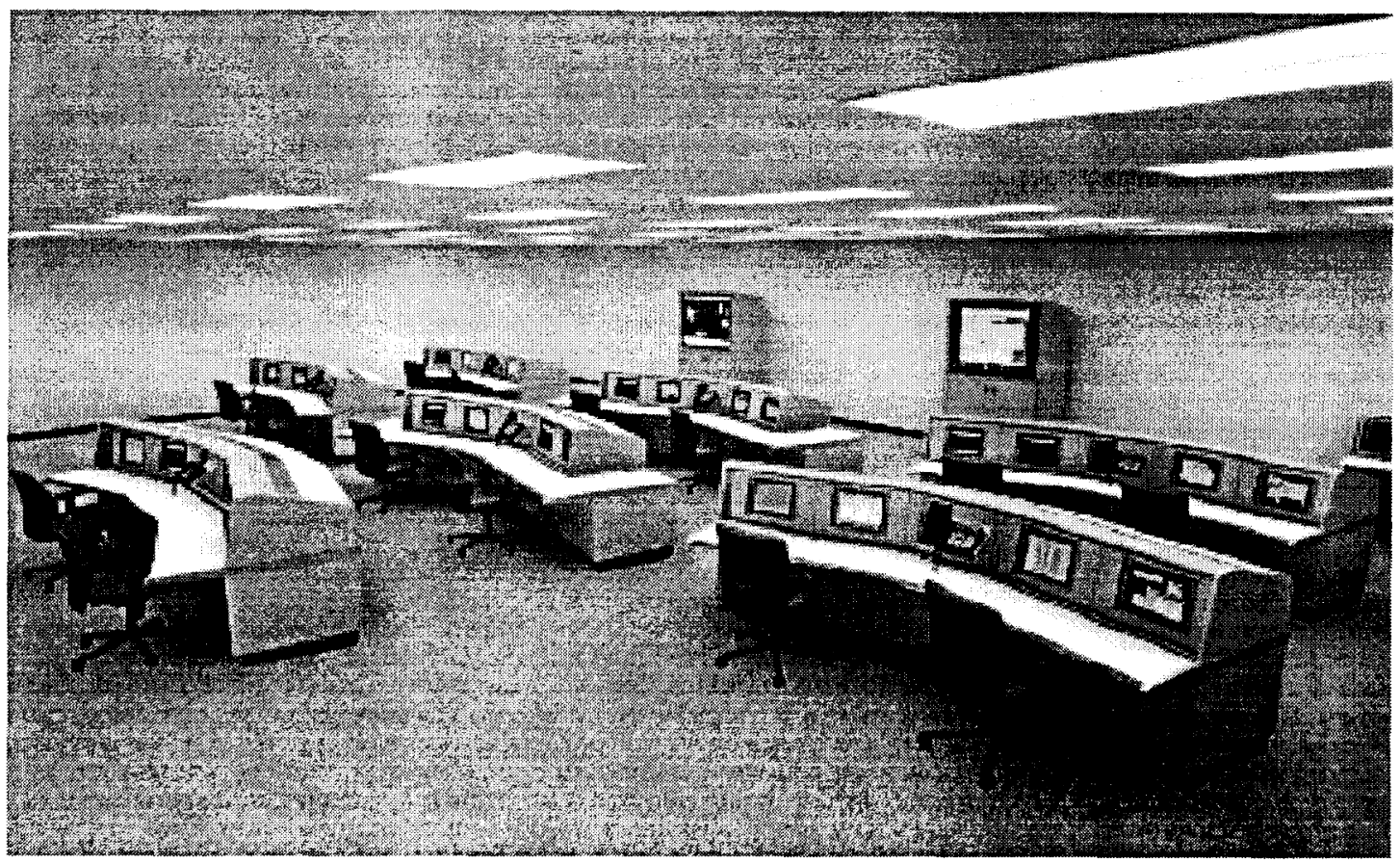

Figure 8 NIF control room

Estimates of the peak traffic requirements for the various subsystems were analyzed as a basis for the network design. The expected peak traffic flows between subsystems in terms of messages per second and message size were specified. This data was combined and analyzed using Excel to determine peak throughputs into and out of each network attached device.

The network design utilizes both Ethernet and Asynchronous Transfer Mode (ATM) technologies to take advantage of the best features of each. ATM provides the connectivity for the systems requiring high-bandwidth or time-sensitive video transport which includes the video FEPs, the operator workstations, and the automatic alignment, master timing, target diagnostics, and optics inspection systems. Ethernet provides connectivity for all other systems, which includes the large majority of systems. Since the ATM and Ethernet attached FEPs will communicate with the operator consoles and the file servers, the operator consoles and file servers have interfaces to both ATM and Ethernet.

The design utilizes $155 \mathrm{Mb} / \mathrm{s}$ ATM (i.e. OC-3c) and Ethernet at both 10 and $100 \mathrm{Mbit} / \mathrm{s}$ speeds, depending on planned traffic requirements. The operator consoles and file servers have $100 \mathrm{Mbit} / \mathrm{s}$ Ethernet connections while the FEPs have 10 or $100 \mathrm{Mbit} / \mathrm{s}$ connections, connected through Ethernet switches. Given the low cost and performance advantages of Ethernet switches relative to shared Ethernet hubs, switches with $100 \mathrm{Mb} / \mathrm{s}$ uplinks will be used. This will keep the message latencies low enough to allow the use of "network triggers", which are time critical. 
A combination of standard Category 5 twisted pair and multi-mode fiber optic cabling is utilized. ATM connections and network links into the computer room are provided over fiber. Fiber is also used for Ethernet connections in areas where electrical noise is a concern and optical isolation is desired (e.g. Power Conditioning and PEPC systems). Twisted pair cabling is used for $10 \mathrm{Mbit} / \mathrm{s}$ Ethernet within a localized set of racks where electrical noise is not a concern.

TCP/IP is the protocol used for reliable data transport between systems, either over Ethernet or ATM. TCP provides retransmission of packets in the event that one is lost or received in error. The only traffic not using TCP will be digitized video and network triggers. Video is transferred using the ATM adapatation layer 5 (AAL5) protocol. Network triggers are sent to many end-nodes simultaneously using multicast protocols.

The ICCS network supports the transport of digitized motion video in addition to the more typical control, status, and shot data. The network transports video images of $640 \times 480 \times 8$ bits/pixel at 10 frames per second between video FEPs (which capture and digitize the camera images) and operator workstations. Each uncompressed video stream requires about 25 Mbit/s of network bandwidth. Each operator workstation can display two video streams, one stream into a window on each of its two displays. Up to 24 video cameras are connected to a single video FEP, and as many as 3 simultaneous video streams are supported from a single FEP (i.e.. $75 \mathrm{Mbit} / \mathrm{s}$ of bandwidth).

Although image decompression for playback is efficient and cost-effective, image compression at the video source is not. Therefore compression is not currently utilized and sufficient network capacity to support uncompressed video is included in the architecture.

Digitized video is sent via the ATM application programming interface (API) using the ATM Quality of Service (QoS) capabilities. The ATM API provides an efficient method of moving large, time-sensitive data streams, resulting in higher frames/sec rates with lower CPU utilization than alternative approaches, which is an important consideration for the video FEPs and console workstations.

It is important that the network provide sufficient capacity to ensure that there is a low loss rate for video data and that the frame jitter (i.e. the interarrival time between image frames) and latency be kept low. If this were not the case, the video display at the workstation would suffer from jerky or delayed playback making it difficult to perform manual control operations. Because the time to retransmit lost packets using TCP is excessive, it is not suitable for use with video traffic. Also, video transmissions need to be multicast, which TCP does not support.

If bandwidth requirements increase in the future, the ICCS network architecture will allow the integration of Gbit/s Ethernet and $622 \mathrm{Mb} / \mathrm{s}$ ATM technologies in a relatively straightforward manner. 
Integrated Computer Controls System Description

This page intentionally left blank. 
Integrated Computer Controls System Description

\section{CORBA Distribution}

Past architectural approaches to distributed controls have relied on the technique of building large application programming interface (API) libraries for each category. The API gives applications access to functions implemented throughout the architecture. This practice results in the programming of large numbers of application interconnections that quickly increase the system complexity, and make software maintenance and evolutionary change much more difficult. A more modern approach to the distributed computing problem is to use a client-server architecture in which software processes communicate using CORBA distribution [4].

CORBA is a standard developed by a consortium of major computer vendors to propel the dominance of distributed objects on local area networks and the worldwide web. The best way to think of CORBA is as the universal "software bus". CORBA is a series of sophisticated, but standard sockets into which software objects can "plug and play" to interoperate with one another. Even when made by different vendors, at different times, the object interfaces are standard enough to coexist and interoperate. By design, CORBA objects operate across languages, operating systems, networks, and tools.

When objects interact it is convenient to label one the client (i.e. the object that initiates the interaction), and the other the server (i.e. the passive object that responds to the initiative). CORBA provides tools for building both clients and servers, and indeed allows an object to be a client in some interactions and a server in others.

At a greatly simplified level, the major parts of CORBA are shown in Figure 9. The interface types and methods provided by the Server Objects and used by the Clients are defined by an industry standard Interface Definition Language (IDL). The IDL compiler examines the interface specification and generates the necessary interface code and templates into which user-specific code is added. The code in the Client that makes use of CORBA objects is written as if the Server was locally available and directly callable -- CORBA takes care of all the rest. 
Integrated Computer Controls System Description

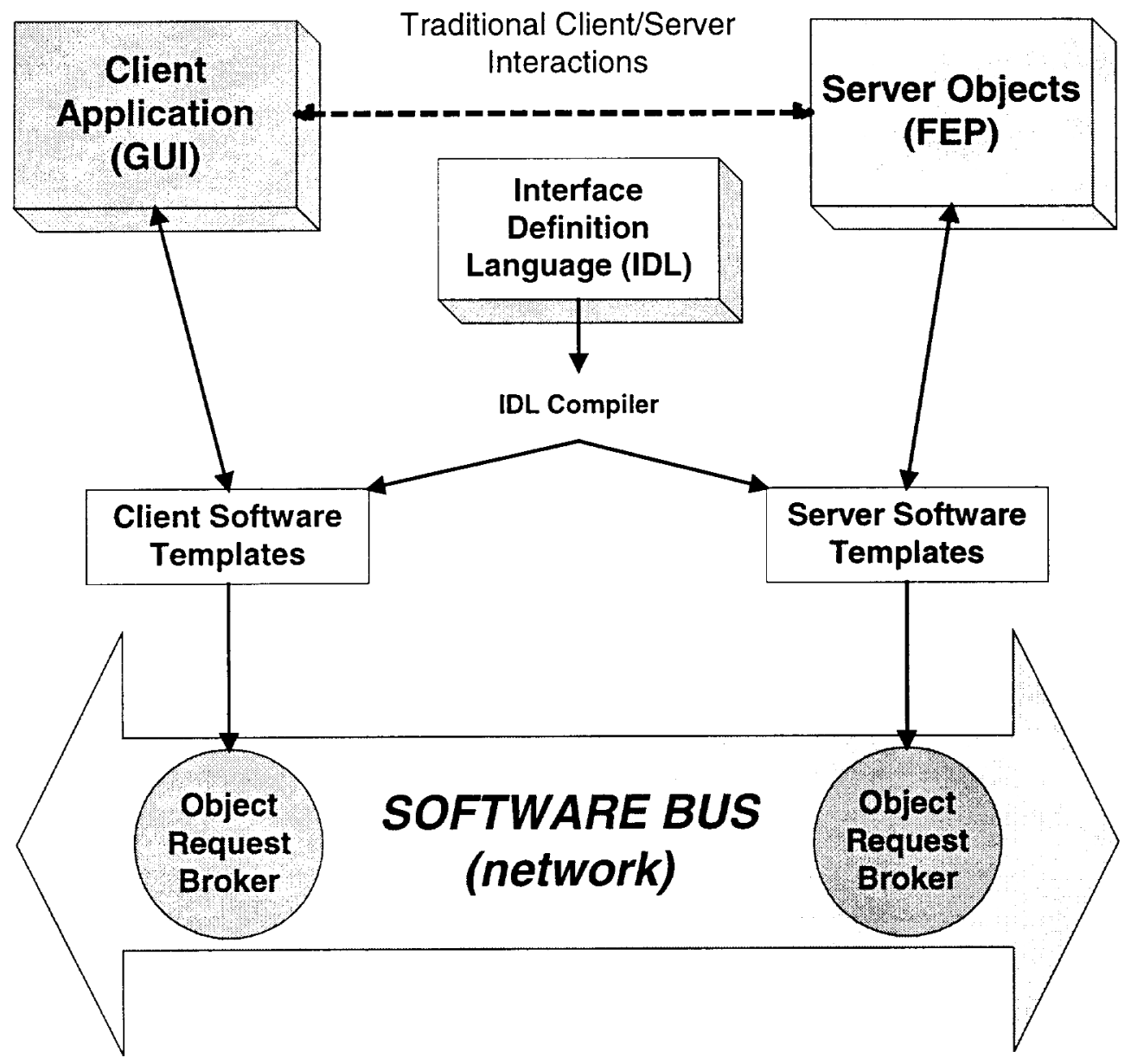

Figure 9 CORBA distribution implements a software bus on the network 
Integrated Computer Controls System Description

\section{ICCS Software Development Environment}

ICCS software development is managed under a software quality assurance plan [5] that covers the entire life cycle of the software design, production, and maintenance. Supporting that plan are software engineering tools that are proven capable of executing large object-oriented projects. Object-oriented analysis and design follow the strategies expressed by Booch [6]. The object-oriented design is captured in the Rose design tool, which maintains schematic drawings of the software architecture.

The tools and the integrated software development process are depicted in Figure 10. Central to the ICCS development are documentation standards for requirement specifications (SRS) and software design descriptions (SDD). These documents are essential to the long-term maintainability of the software in view of periodic software upgrades and staffing turnover expected during the 30-year life of the NIF.

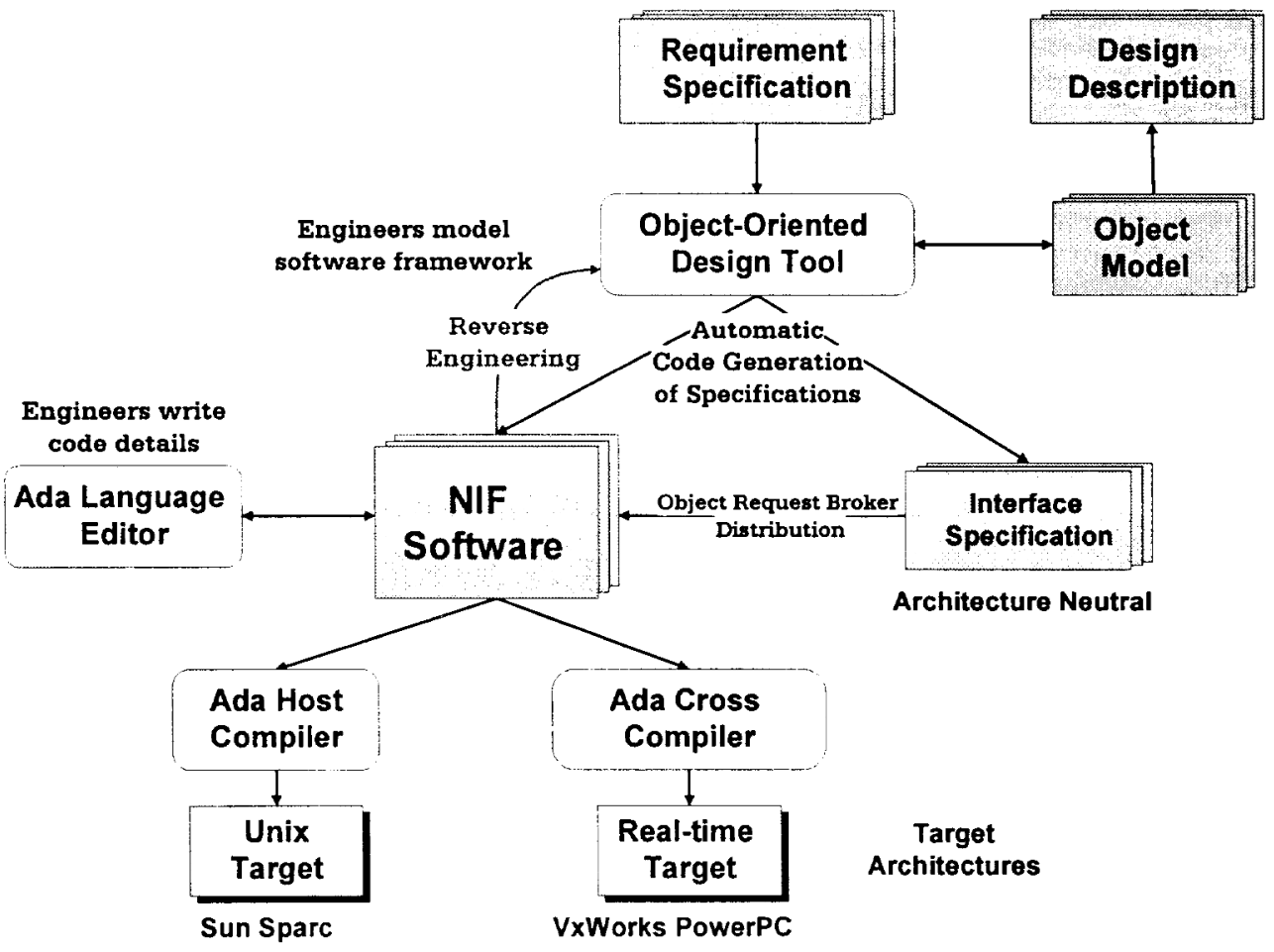

Figure 10 Flowchart of the ICCS software engineering process

Detailed requirements expressed in the SRS are analyzed and result in the objectoriented design maintained by the Rose tool. Classes are defined to implement the responsibilities of the software. Important software use cases are expressed in terms of object scenario diagrams. In general terms, Rose is used to model the interfaces and interactions between major software entities in the design. After the modeling 
cycle is complete, the Rose tool generates the Ada specifications corresponding to the class interface and type definitions. The developer fills in the detailed coding necessary to implement internal details of each class. Classes that are distributed generate IDL, which is passed through the IDL compiler to generate Ada code as before. The SDD is a narrative document for explaining the object-oriented model and contains other design material necessary for implementing the software.

The Ada source code then takes one of two paths depending on the target processor architecture. For Solaris, the source is compiled, linked, debugged, and tested on the workstation. For PowerPC or other VxWorks targets, the source is compiled and debugged under the Apex Works cross-compilation system. In either case, the models, sources, binaries, and run-time images are managed by the Apex configuration management system, which provides full capability for version control. Using Apex subsystem facilities, the frameworks can be independently developed by different engineers, each of which has a protected view of the other components.

The ICCS incorporates Ada, CORBA, and object-oriented techniques to enhance the openness of the architecture and portability of the software. As a consequence, many alternate implementation choices exist now and more will surface in the future. Notable examples of interesting tools include Windows NT and the Intel family of processors. At the current stage of the project, the tools used to construct the ICCS are indicated in Table 2. 
Integrated Computer Controls System Description

\begin{tabular}{l|l}
\multicolumn{1}{c|}{ Tool } & \multicolumn{1}{c}{ Vendor \& type } \\
\hline UNIX OS and workstations & Sun Solaris on UltraSparc \\
\hline Real-time operating system & Windriver VxWorks / Tornado \\
\hline FEP CPU chipset & Motorola PowerPC family \\
\hline Ada 95 host compiler & Rational Ada (Solaris) \\
\hline $\begin{array}{l}\text { Alternate Ada 95 host } \\
\text { compiler }\end{array}$ & GNAT Ada (Solaris) \\
\hline Ada 95 cross compiler & Rational Apex Works (VxWorks) \\
\hline C++ host compiler & Rational C++ \\
\hline Configuration management & Rational Apex \\
\hline $\begin{array}{l}\text { CORBA on UNIX and } \\
\text { VxWorks }\end{array}$ & $\begin{array}{l}\text { Objective Interface Systems } \\
\text { ORBexpress }\end{array}$ \\
\hline Relational and object database & Oracle \\
\hline Graphical user interface & UIM/Orbix, Tcl/Tk - to be specified \\
\hline Object-oriented modeling & Rational Rose / Ada
\end{tabular}

Table 2 Tools used in the construction of the ICCS software 
Integrated Computer Controls System Description

This page intentionally left blank. 


\section{Reusable Supervisory Software Framework}

The ICCS supervisory software framework is a collection of collaborating abstractions that are used for the construction of the application software. Frameworks [7] reduce the amount of coding necessary by providing pre-built components that can be extended to accommodate specific additional requirements. The framework also promotes code reuse by providing a standard model and interconnecting backplane that is shared from one application to the next. The framework concept enables the cost-effective construction of the NIF software and provides the basis for long-term maintainability and upgrades.

Components in the ICCS framework plug into the CORBA bus, as shown in Figure 11 . Using this very simple view of the components, a few scenarios can be described. Graphical user interface works with status report to provide operator controls by collaborating with device control and status monitor objects located in the front end processor. Archive (and a commercial database) adds the services needed for experimental data processing and trending of machine performance. Configuration interacts with object factories to create, locate, and coordinate the software objects that implement device services within the FEP. Other components (not shown in the figure) provide event logging and process interlock services.

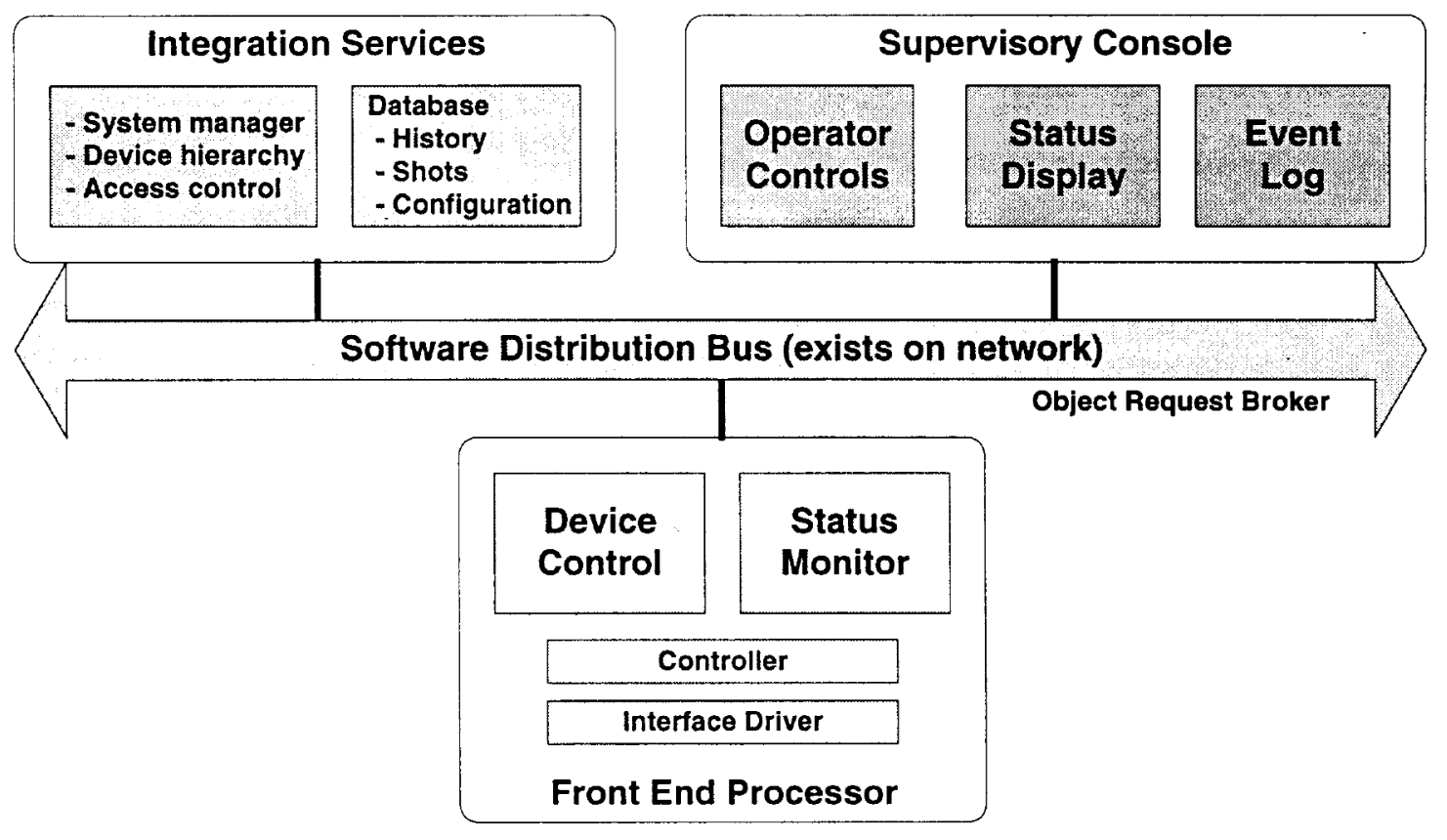

Figure 11 ICCS framework objects plug into the CORBA software bus 
An example of two objects that collaborate is the interaction between a status monitor object that watches a motor device and informs a particular status report object when the motor moves. The status report (on the supervisory workstation) is responsible to collect the stream of status updates from the status monitor (on the FEP) and make them available to the operators. The status report object updates a graphic user interface so an operator can see the current status of the motor device. This scenario is an example of two objects that work together smoothly even though they are on different computers.

The following subsections introduce the ten frameworks that form the basis of the ICCS supervisory software.

\subsection{Configuration}

Configuration is a hierarchical organization for the static data that define the hardware control points that are accessible to the ICCS. Configuration provides a taxonomic system that is used as the key by which clients locate devices (and other software services) on the CORBA bus; the configuration hierarchy is the unique source of device identifying information within the ICCS. During normal operation, configuration provides to clients the CORBA references to all distributed objects. A useful feature of configuration is the ability to select entire branches of the hierarchy for subsequent operations.

The naming convention for NIF devices is described in reference [8]. A taxon is the hierarchical name given to nodes in the configuration data structure and has the general form:

\section{Taxon $\Rightarrow$ (Location, Unit, Component, Identifier).}

An important responsibility of configuration is the initialization of front-end processors during start-up. Configuration data are stored in the database and describe how and where the control hardware is installed in the system. Calibration data for sensors, setpoints for alignment devices, and I/O channels used by devices on interface boards are examples of static data managed by configuration. During ICCS start-up, this framework collaborates with an object factory located in the FEP. Using the data and methods stored in the configuration database, the object factory instantiates, initializes, and determines the CORBA reference for each device and controller object in the FEP.

\subsection{Status Monitor}

The status monitor provides generalized services for broad-view operator display of device status information using the push model of event notification. The status monitor observes devices and notifies other parts of the system when the status changes by a significant amount.

The status monitor assures that timely status is available to the supervisory layer from thousands of devices, while economizing on network traffic. Economy is 
achieved by periodically polling devices at sensible rates with a monitor object located in the FEP; network messages are only generated when changes of interest occur. Furthermore, status information from each FEP is aggregated into a single network message for transmission to the client in the supervisory workstation. Monitor objects have periodic rates typically ranging from 0.1 to 10 seconds. A filtering mechanism is provided for managing the change threshold.

\subsection{Sequence Control Language}

The sequence control language (SCL) framework is used to create custom scripting languages for the NIF applications. The SCL service automates sequences of commands executed on the distributed control points or other software artifacts. Each SCL instance provides access to a subset of the ICCS device control and status methods available for a particular application domain area. Script languages are implemented internally with the Tool Command Language (Tcl) interpreter.

The SCL provides the following control constructs:

- Serial execution of commands either inside or outside of control constructs

- Indeterminate loops (while-true-loop)

- Conditional branching (if-then-elsif-else-endif)

- Synchronized concurrent threads of control (fork-join)

- Passing of user-supplied arguments to user-supplied methods at run-time

- Dispatching call to user-supplied method

Operators create and edit sequences by selecting icons that represent control constructs, Boolean functions, and user-supplied methods from a visual programming palette. The icons are then interconnected to program the sequence and any Boolean conditions or method arguments needed are defined to complete the sequence script. Examples include a checklist SCL for use by Laser Diagnostics operators and an embedded SCL for closed-loop processing in the Automatic Alignment FEP.

\subsection{Graphical User Interface}

All human interaction with the ICCS will be via graphical user interfaces displayed upon control room consoles or on $X$ Terminals distributed throughout the facility. The GUI is implemented as a framework in order to ensure consistency across the applications. The GUI framework is based upon the X Windows system and Motif policies. Commercial GUI development tools are used to construct the display graphics. In addition, the color scheme used on Nova is incorporated to provide continuity to operators as they migrate from that system to the NIF. 
This framework consists of guidelines for look and feel as well as some common graphical elements:

- NIF Control Panel

- Beam Selector

- Laser Map

- Status Summary

- Countdown clock

Examples of the common graphical elements are shown in Figure 12, Figure 13, and Figure 14.

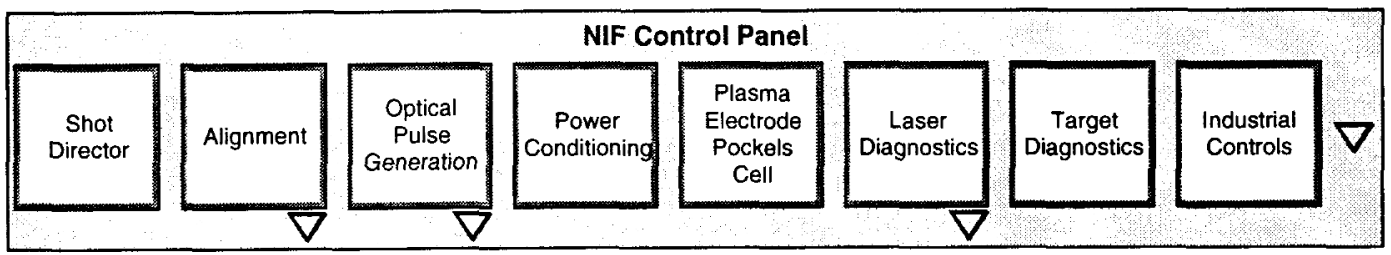

Figure 12 Top-level subsystem access panel
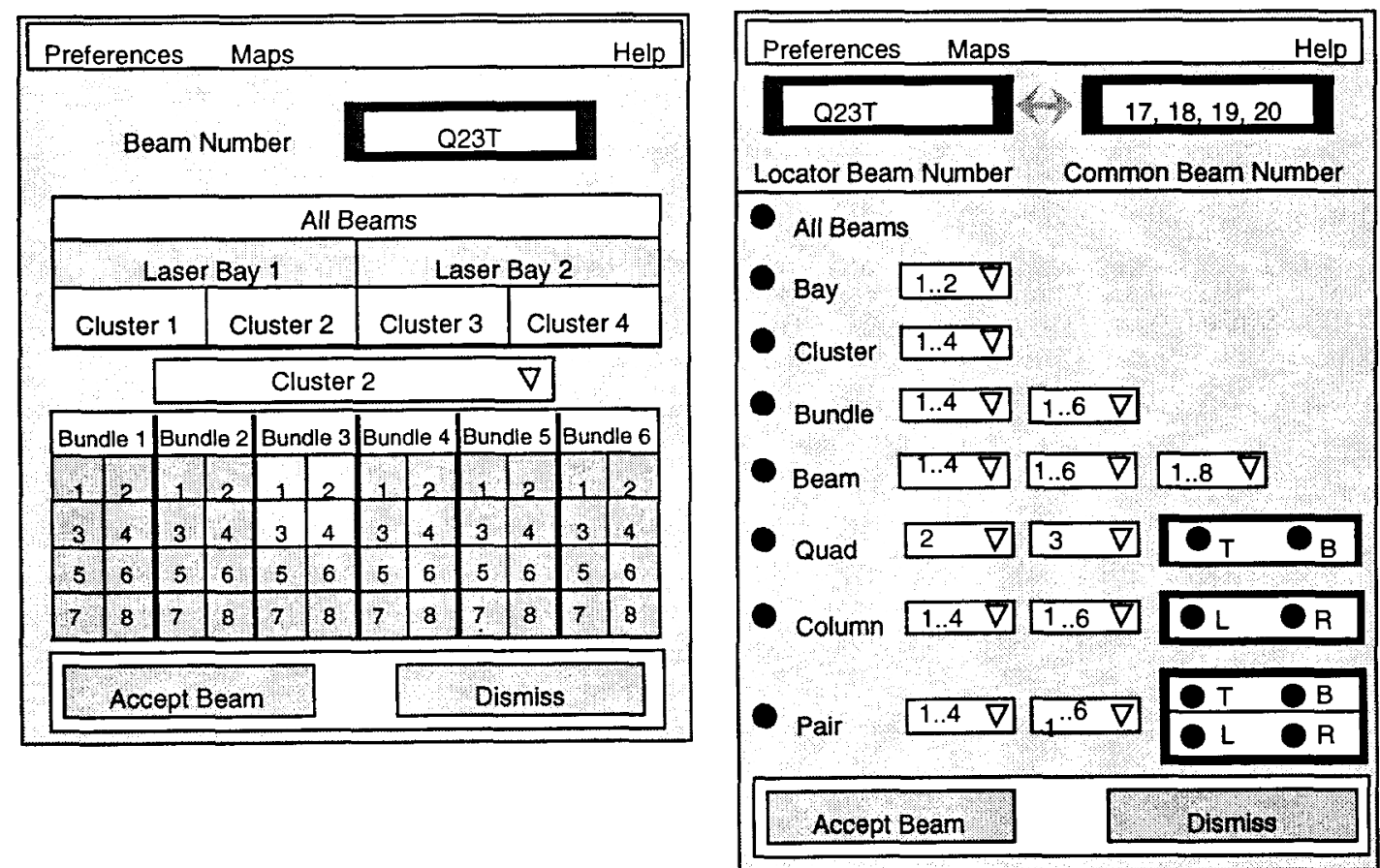

Figure 13 Common beam selector GUI 


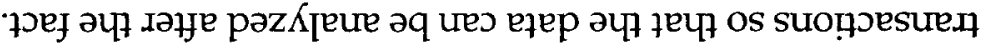

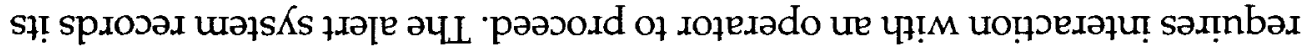

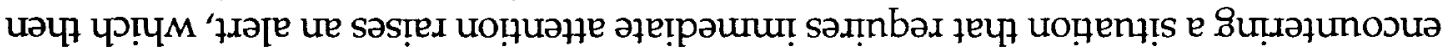

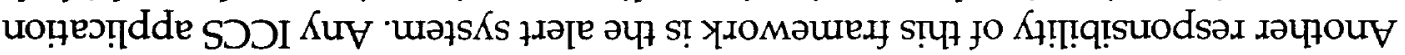

s8oI

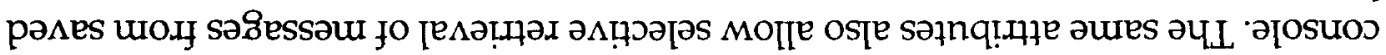

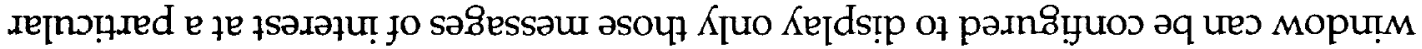

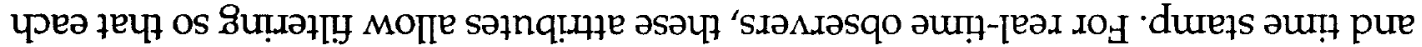

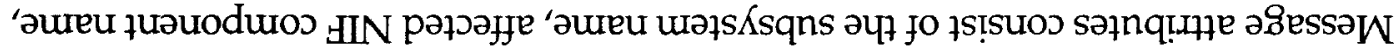

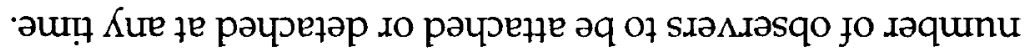

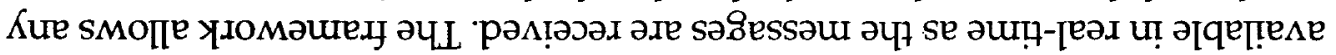
әле suoṭe

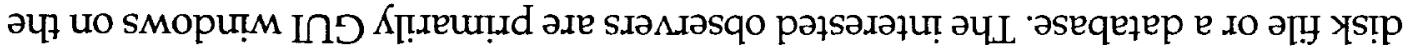

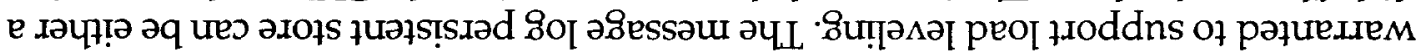

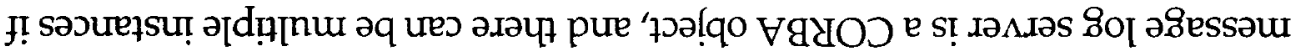

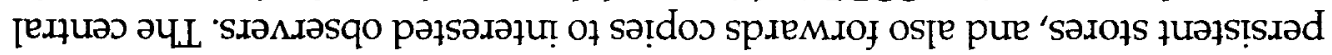

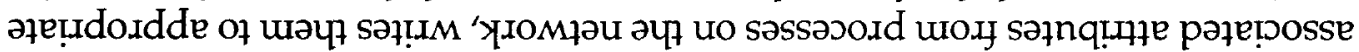

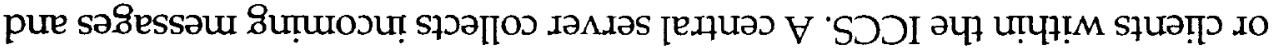

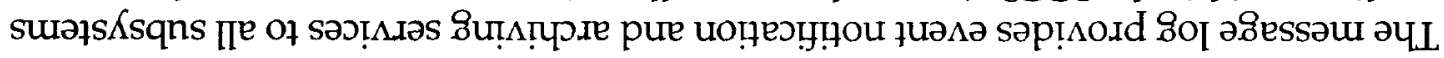

607 ə6essaw $9 " 2$

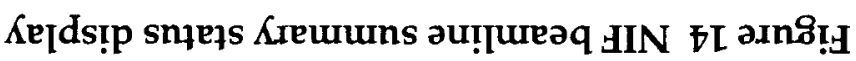

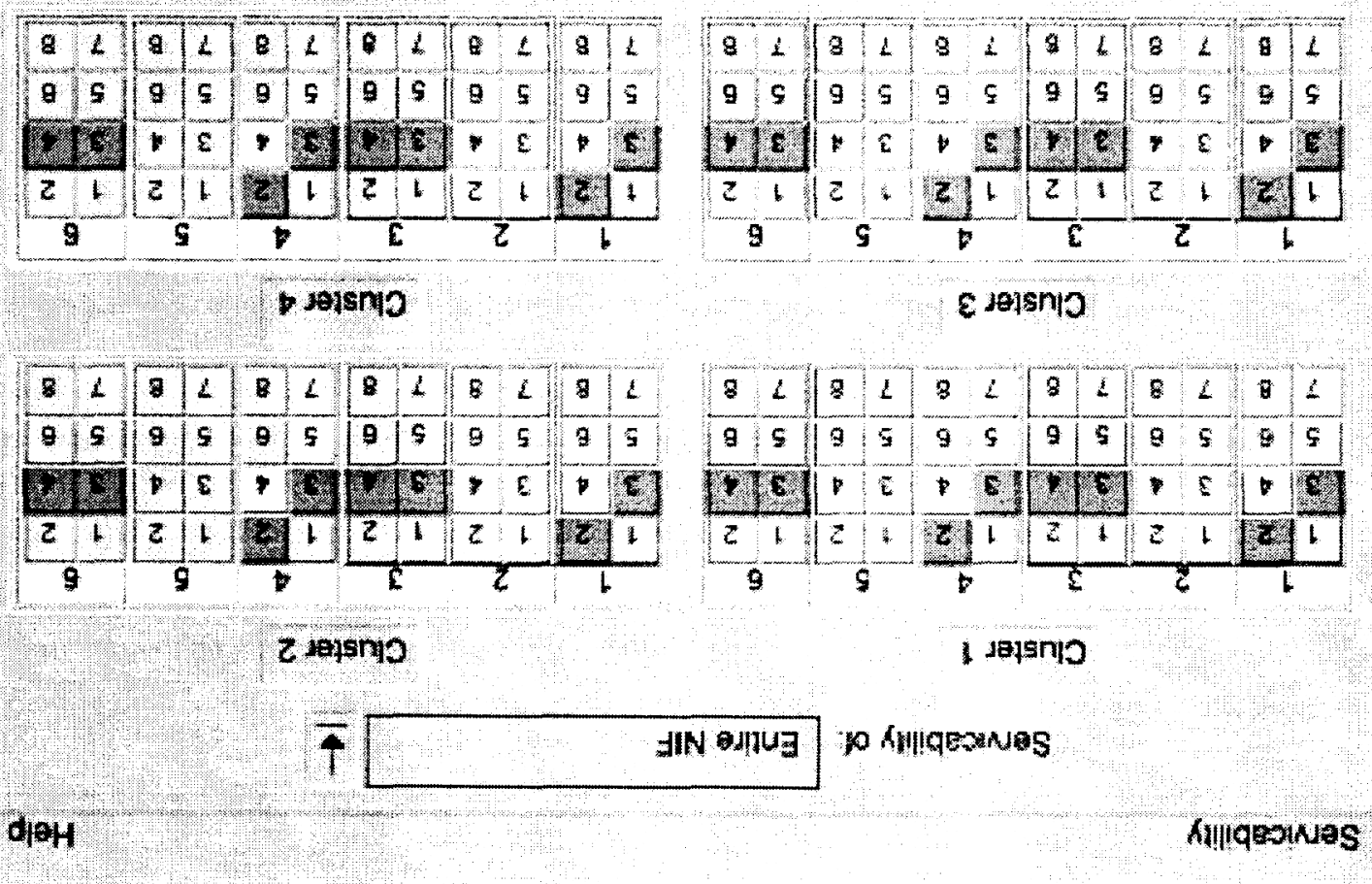

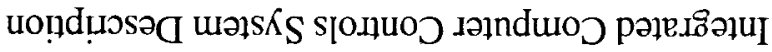


Integrated Computer Controls System Description

\subsection{Reservations}

The reservation framework manages access to devices by giving one client exclusive rights to control or otherwise alter the device. The framework uses a lock-and-key model. Reserved devices that are "locked" can only be manipulated if and when a client presents the "key". Provision is made to allow an entire sub-tree from the configuration hierarchy to be reserved in one operation. Additionally, provision is made to construct groups of devices for convenient reservation. Read access to obtain status is not affected by the reservation.

As an example, the reservation service coupled with sequence control is typically used to implement machine process interlocks. These are specific configurations of the laser- and target-area hardware imposed to prevent damage to equipment (not related to personnel safety) or loss of shot data. Sequence control reserves needed devices and executes functions using the devices to establish conditions needed. Further access is prevented by the reservation service until the devices are released.

\subsection{System Manager}

The system manager provides services essential for the integrated management of the ICCS network of hundreds of computers. This component ensures necessary processes and computers are operating and communicating. Services include parameterized system start-up, shutdown, and process watchdog monitoring. An important responsibility is coordinating the sequence of restarting portions (or all) of the ICCS computer system. FEPs can be started in either networked or stand-alone modes.

System manager is distributed; a central system manager expects periodic reports from system managers located in each workstation and FEP as to the operating status of the processes on each machine. The central system manager maintains a central view of the entire ICCS, and also collects metrics from the computers and network. This data may be reviewed to evaluate overall ICCS performance. Clients subscribe to the central system manager in order to be notified when a given processor or process fails, or changes in a significant way.

\subsection{Machine History Archive}

Machine history is information about the performance and operation of the NIF that originates within the ICCS. The history archive collects data for analysis of the NIF operation in order to improve efficiency and reliability. Some information is also copied to the facility product data management (PDM) system to support the reliability, availability, and maintainability (RAM) goals of the facility. 
Examples of such information are:

- Installation and service of components

- Occurrences of abnormal conditions

- Records of operating service time or usage count

- Periodic readings of facility and environmental monitoring sensors

- Alignment reference images

- Experimental setup configurations

This framework allows different applications to define history records as extensible objects and to build histories as constantly growing series of history records. Any ICCS component can instantiate services from the history archive framework. History records are extensible to allow more attributes to be included in later history records as requirements evolve. The framework supports the retrieval of multiple histories of large groups of objects so that, for example, one can generate a report on the performance of all motors for studying reliability and predicting preventive maintenance.

\subsection{Generic Front End Processor}

The generic FEP framework pulls together the distributed aspects of the other frameworks (in particular the system manager, configuration, status monitor, and reservation frameworks) by adding unique classes for supporting device and controller interfacing. These classes are responsible for hooking in CORBA distribution as well as implementing the creation, initialization, and connection of device and I/O controller objects. The generic FEP also defines a common hardware basis including the target processor architecture, backplane, I/O boards, device drivers, and field bus support. The FEP application developer extends the base classes to incorporate specific functionality and state machine controls.

\subsection{Shot Data Archive}

The ICCS is not responsible for the permanent storage (archive) or in-depth study of shot data; it is however responsible for collecting the data from the diagnostics, making the data immediately available for "quick look" analysis, and delivering the data to an archive. The framework contains a server working in collaboration with the system manager to assure that requested shot data are delivered to a disk staging area. The archive server is responsible for building a table of contents file and then forwarding the table and all data files to the archive.

An important aspect of this framework is that all data files will be written in one of a few approved formats. In addition to text files, the most favored format will be HDF files. Many commercial off-the-shelf visualization tools (such as Interactive Data Language) can read this self-describing data format, so the framework saves physicists from having to translate data formats before viewing their results. The definition of shot data includes not only the target diagnostics data but also timing and laser diagnostics data that will help experimentalists interpret their results. 
Integrated Computer Controls System Description

This page intentionally left blank. 


\section{ICCS Databases}

The ICCS has many complex data structures to represent the "real world" components that must be controlled, monitored, and stored. Figure 15 depicts the development process for database design and application software. Application requirements are modeled and subsequently built into code. Database requirements are modeled with entity relationship diagrams and subsequently defined in data definition language to a database management system (DBMS).

Database servers provide the run-time interface between the DBMS and the ICCS application programs. The servers hide the DBMS specifics from the application and implement a consistent interface. A database server is built for each identified category of data to be managed by the ICCS. These categories of data are discussed in further detail below. The Standard Query Language (SQL) is used as the interface between the database servers and the DBMS. SQL is the standard interface language for most commercial database management products. Use of SQL serves to insulate the ICCS from changes in the database management system.

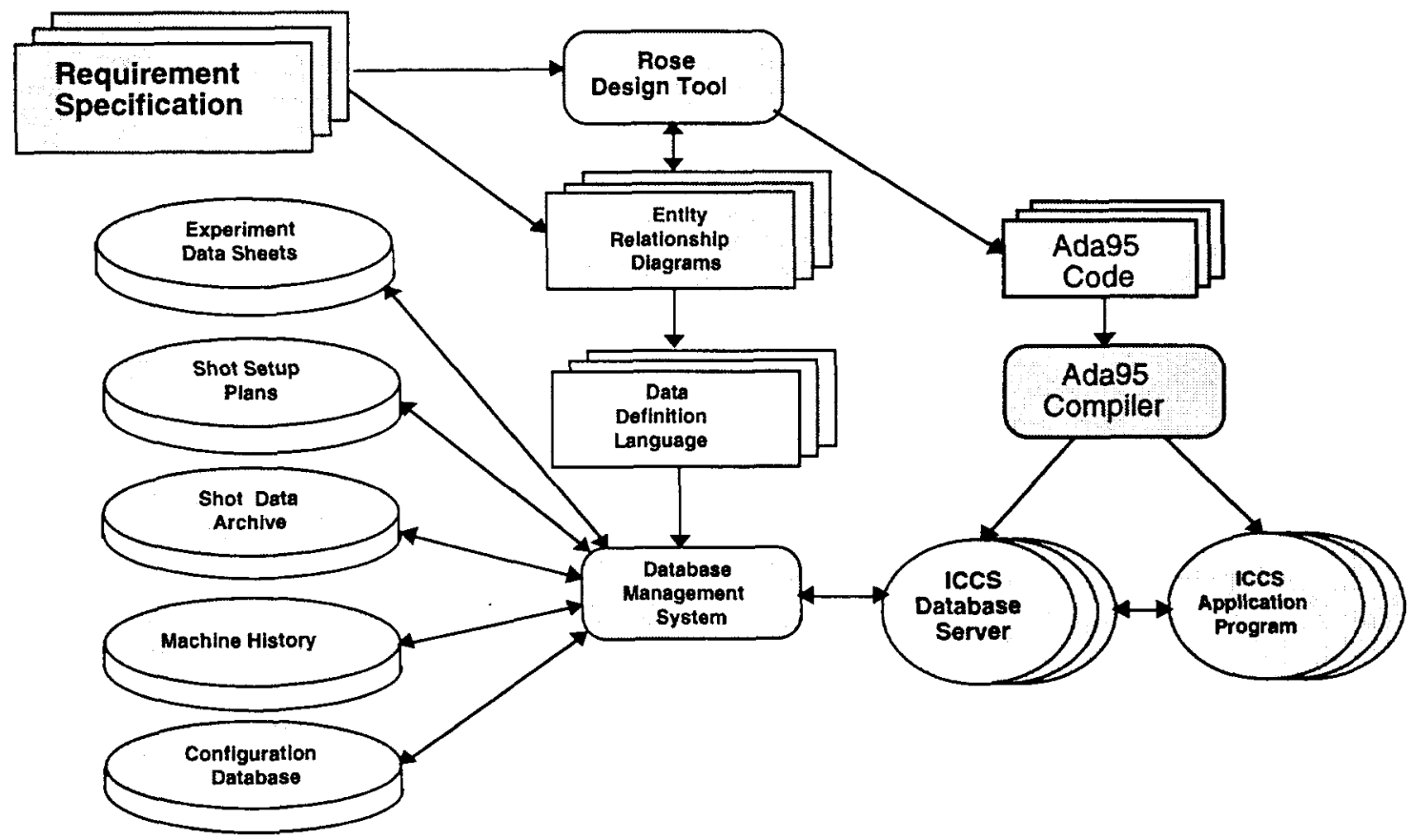

Figure 15 ICCS Databases 
Communication between the application programs and the database servers is accomplished using the CORBA software bus as depicted in Figure 16. CORBA generalizes access to software objects in the client/server network.

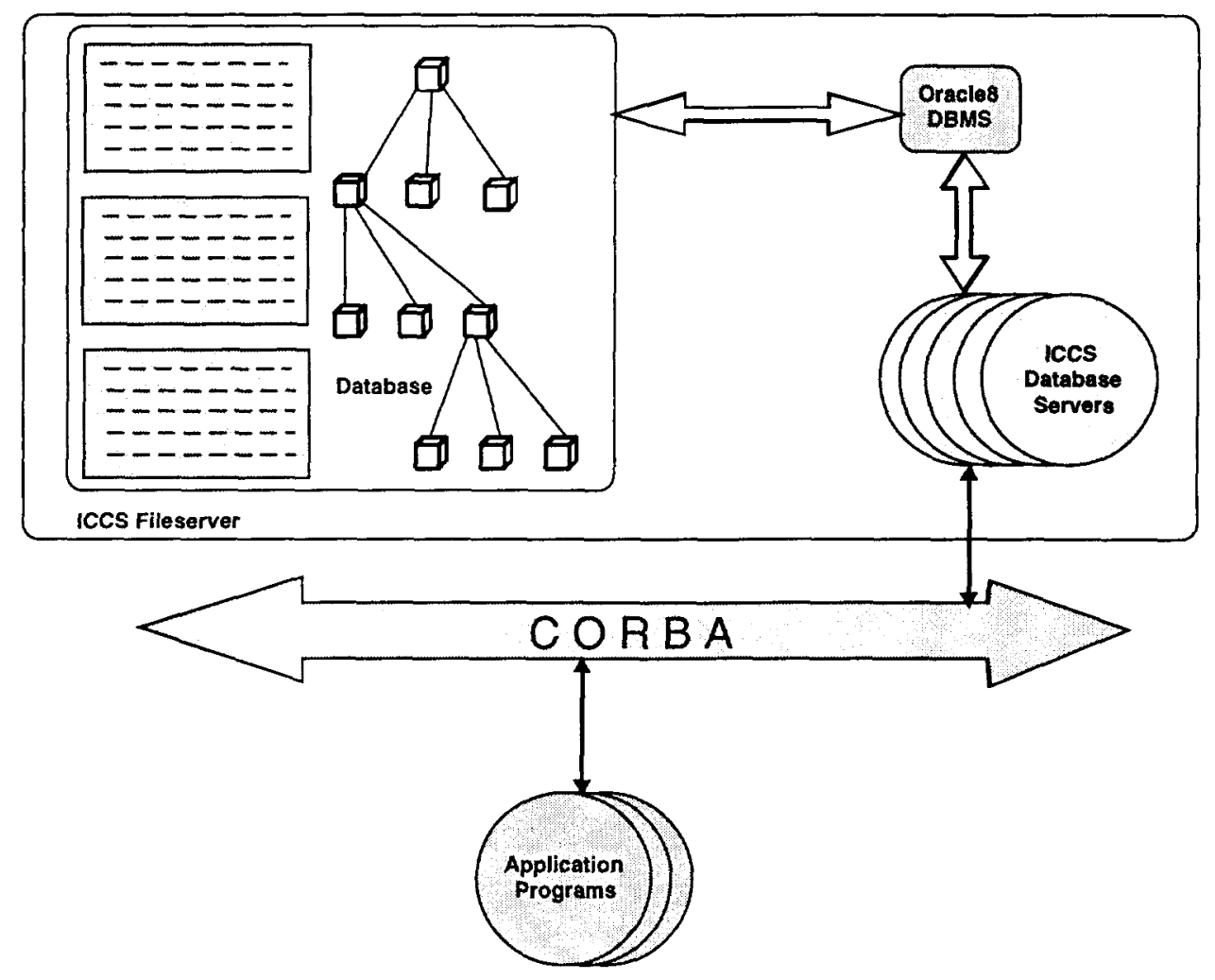

Figure 16 Database interprocess communication

The ICCS databases hold information for several different purposes that can be classified as different database management problems as follows:

- Large vector or image data - the UNIX file system provides the services of storage and access to files for data analysis programs. The data analysis programs qualify as "no query" applications and operate on files that are a sequence of bytes of arbitrary length. Current database technology allows for storing and/or cataloging arbitrarily large files in the database. A catalog scheme is used to keep track of image data files.

- Tabular data with queries - a relational DBMS provides the standard query language and client tool kits. The relational data model provides a wide variety of data query and aggregation mechanisms which are important for ad hoc historical analysis of shot archive information. 
Integrated Computer Controls System Description

- Complex data without queries - an object-oriented DBMS is well suited for management of complex data. An Object DBMS allows the description of control system objects and their persistent data identically to how they appear in the run-time control system. Access to this information occurs at object creation time when the control system starts up, and updates are performed through custom interfaces needing little or no query capabilities. The database objects are defined as part of the object-oriented software design process.

The data management requirements for the ICCS encompass all of these classes of data. The Oracle8 "object-relational" database management system (O-RDBMS) was chosen to address the ICCS database problem domain. Oracle8 supports user defined data types (objects), scaleable on-line transaction processing (OLTP) performance, large object storage, and very large databases (> 100Gbytes). The control system architecture is based upon object technology and will need objectoriented persistence to support its initialization and operation. In addition, the shot data archive and machine history components of the ICCS will use a combination of relational, object, and large-object features.

Five major categories of data have been identified, which are termed application databases. A brief description of each application database is given below. Complete design information is given in the Software Frameworks volume of the ICCS Software Design Descriptions.

\subsection{Configuration Database}

The configuration database contains the persistent data associated with the software objects that comprise the as-built control system. Examples include sensor calibration data, optical component characteristics, and device initialization data. Device initialization includes hardware location, CPU, bus address, channel, etc. This persistent data is generally extracted from the configuration database when an object is created. The objects in the configuration database map directly to software objects that implement the control system. This makes a common view of software objects and their associations in the object hierarchy for both persistent storage and run-time implementation. To obtain this common view, the object-oriented features of the DBMS allows the description complex objects and their relationships. The configuration database is generally accessed once for each object at object creation time (e.g. to create control point objects in the FEP during start-up) when the configuration data is loaded into the running system.

\subsection{Experiment Data Sheets}

An experiment data sheet typically identifies a target diagnostic and a desired set of inputs that can be measured by the diagnostic. Each active diagnostic on a shot has a corresponding experiment data sheet. The experimenter builds experiment data 
sheets that subsequently are to be allocated to specific shots. A single NIF shot may accommodate multiple experiment data sheets.

\subsection{Shot Setup Plan}

The shot setup database contains desired shot characteristics and the configurable parameters for the devices that will be used in a shot. When a device is to be configured for a specific shot, the desired shot characteristics are analyzed to determine the necessary settings. These settings are communicated to the device during shot preparation. For example, laser diagnostics beam power balancing uses a model of the laser energy gain profile to calculate settings for devices to be used on a laser shot of a specific desired energy. The calculated settings from this model are stored in the shot setup database and extracted when the actual devices are configured to those settings. These data, along with other information describing the machine configuration and operation, are archived along with actual shot data for future analysis.

\subsection{Shot Archive Database and Shot Archive Files}

The Shot Archive Database contains a snapshot of the control system objects at and near shot time. This includes the shot setup database and any shot data acquired as a result of the shot. Each ICCS supervisory application has some form of data archiving process. The archiving process gathers subsystem results and stores them in the shot archive database and shot archive files. The shot data are used in off-line analyses to fully characterize the performance of NIF experiments. Image data and other large-array data are stored in shot archive files and accessed by various off-line data analysis programs. The shot archive files use standard, common file formats such as HDF.

\subsection{Machine History}

The machine history database tracks the operational history of component performance and assists with maintenance activities. Periodic maintenance (PM) schedules are required for many components and the machine history database will trigger reports that notify operations staff of any required PM activities. The maintenance schedules and procedures for maintenance are contained in the machine history database. Component failures and usage history are stored for failure mode analysis and reliability, availability, and maintainability (RAM) statistics. 
Integrated Computer Controls System Description

\section{Front-End Processors}

Several kinds of front-end processor are used in the ICCS to control the NIF hardware and interface to the supervisory control system. The various FEP (summarized in Table 3) are discussed in the following sections.

\begin{tabular}{|c|c|c|c|c|c|}
\hline Section & $\begin{array}{l}\text { Front End } \\
\text { Processor }\end{array}$ & Qty & $\begin{array}{l}\text { Operating } \\
\text { System }\end{array}$ & Control Points Summary & $\begin{array}{c}\text { Communication } \\
\text { Notes }\end{array}$ \\
\hline 9.1 & $\begin{array}{l}\text { Master Oscillator } \\
\text { Room }\end{array}$ & 2 & VxWorks & $\begin{array}{l}\text { Waveform generator, energy meter, } \\
\text { power meter, sampling scope, wave } \\
\text { meter, programmable power supply }\end{array}$ & GPIB, RS485 \\
\hline 9.2 & Preamplifier Module & 48 & VxWorks & $\begin{array}{l}\text { Stepmotor, timing, digital } 1 / O \text {, pulse } \\
\text { power, vacuum, thermal control, } \\
\text { interlocks }\end{array}$ & $\begin{array}{l}\text { RS232, } \\
\text { LONworks }\end{array}$ \\
\hline 9.3 & $\begin{array}{l}\text { Plasma Electrode } \\
\text { Pockels Cell }\end{array}$ & 4 & VxWorks & $\begin{array}{l}\text { Digital } 1 / O \text {, analog } \mathrm{I} / \mathrm{O}, \mathrm{RF} \text { digitizer, RF } \\
\text { multiplexor, gas control, vacuum, } \\
\text { pulse generator, valves, embedded } \\
\text { controllers, power supplies }\end{array}$ & $\begin{array}{l}\text { Ethemet, } \\
\text { RS485, RS232 }\end{array}$ \\
\hline 9.4 & Power Conditioning & 4 & Solaris & Embedded controller & Ethemet \\
\hline 9.4 & $\begin{array}{l}\text { Power Conditioning } \\
\text { Embedded }\end{array}$ & 192 & VxWorks & $\begin{array}{l}\text { Pulse power, digital I/O, timing, high } \\
\text { voltage, current monitor, dump, } \\
\text { trigger, gas control, transient digitizer }\end{array}$ & Ethernet \\
\hline 9.5 & Integrated Timing & 26 & VxWorks & $\begin{array}{l}\text { Delay generator, time interval meter, } \\
\text { digital } 1 / O \text {, multiplexor, digital clock }\end{array}$ & $\begin{array}{l}\text { GPIB, Fast } \\
\text { Ethernet }\end{array}$ \\
\hline 9.6 & Automatic Alignment & 2 & Solaris & $\begin{array}{l}\text { Uses alignment controls, video } \\
\text { distribution }\end{array}$ & ATM \\
\hline 9.7 & Video Distribution & 31 & Solaris & Frame grabber, network trigger & ATM \\
\hline 9.8 & Industrial Controls & 2 & Solaris & $\begin{array}{l}\text { PLC, vacuum, gas, oxygen, } \\
\text { temperature, humidity, access control, } \\
\text { digital } 1 / \mathrm{O}, \mathrm{pH} \text {, moisture alam, hazard } \\
\text { display, safety system, environmental } \\
\text { protection }\end{array}$ & $\begin{array}{l}\text { Ethernet, Allen } \\
\text { Bradley } \\
\text { Controlnet }\end{array}$ \\
\hline 9.9 & Alignment Controls & 124 & VxWorks & Stepmotor, binary actuator, digital l/O & Ethemet \\
\hline 9.10 & Optics Inspection & 3 & Solaris & CCD camera & ATM \\
\hline 9.10 & $\begin{array}{l}\text { Scientific Grade } \\
\text { Cameras }\end{array}$ & 2 & Windows NT & High-resolution CCD camera & Ethemet \\
\hline 9.10 & Streak Camera & 6 & Windows NT & CCD camera & Ethernet \\
\hline 9.11 & $\begin{array}{l}\text { Laser Energy } \\
\text { Diagnostic }\end{array}$ & 6 & VxWorks & $\begin{array}{l}\text { Charge-integrated photodiode, } \\
\text { calorimeter }\end{array}$ & $\begin{array}{l}\text { Ethernet, } \\
\text { LONworks }\end{array}$ \\
\hline 9.12 & $\begin{array}{l}\text { Laser Power } \\
\text { Diagnostic }\end{array}$ & 4 & Solaris & $\begin{array}{l}\text { Transient digitizer, programmable } \\
\text { power supply, vacuum photodiode }\end{array}$ & $\begin{array}{l}\text { Ethemet, MXI } \\
\text { bus }\end{array}$ \\
\hline 9.13 & Wavefront Control & 24 & VxWorks & Deformable mirror, Hartmann sensor & Fast Ethernet \\
\hline 9.14 & Target Diagnostics & 2 & tbd & $\begin{array}{l}\text { Diagnostic instrument manipulator, } \\
\text { time-resolved X-ray imaging, } X \text {-ray slit } \\
\text { streak camera, static X-ray imaging, } \\
\text { others tbd }\end{array}$ & Ethernet \\
\hline & Total Processors & 482 & & & \\
\hline
\end{tabular}

Table 3 Summary of front-end processors

\subsection{MOR FEP}

The Master Oscillator Room (MOR) FEPs [Figure 17] are primarily responsible for controlling and monitoring the generation of the optical pulse that feeds the NIF laser amplifier chain. The master oscillator consists of a single diode pumped fiber optic ring laser whose output pulse is shaped into a $30 \mathrm{~ns}$ long pulse, and then phase modulated by $3 \mathrm{GHz}$ to prevent SBS and $17 \mathrm{GHz}$ for SSD. This pulse is then optically amplified and split into 48 separate channels, which are then amplitude 
modulated. The pulse repetition rate in the MOR is $960 \mathrm{~Hz}$, and it operates continuously. The seed pulses from the MOR are fed over PM fiber cables to each of the PAMs.

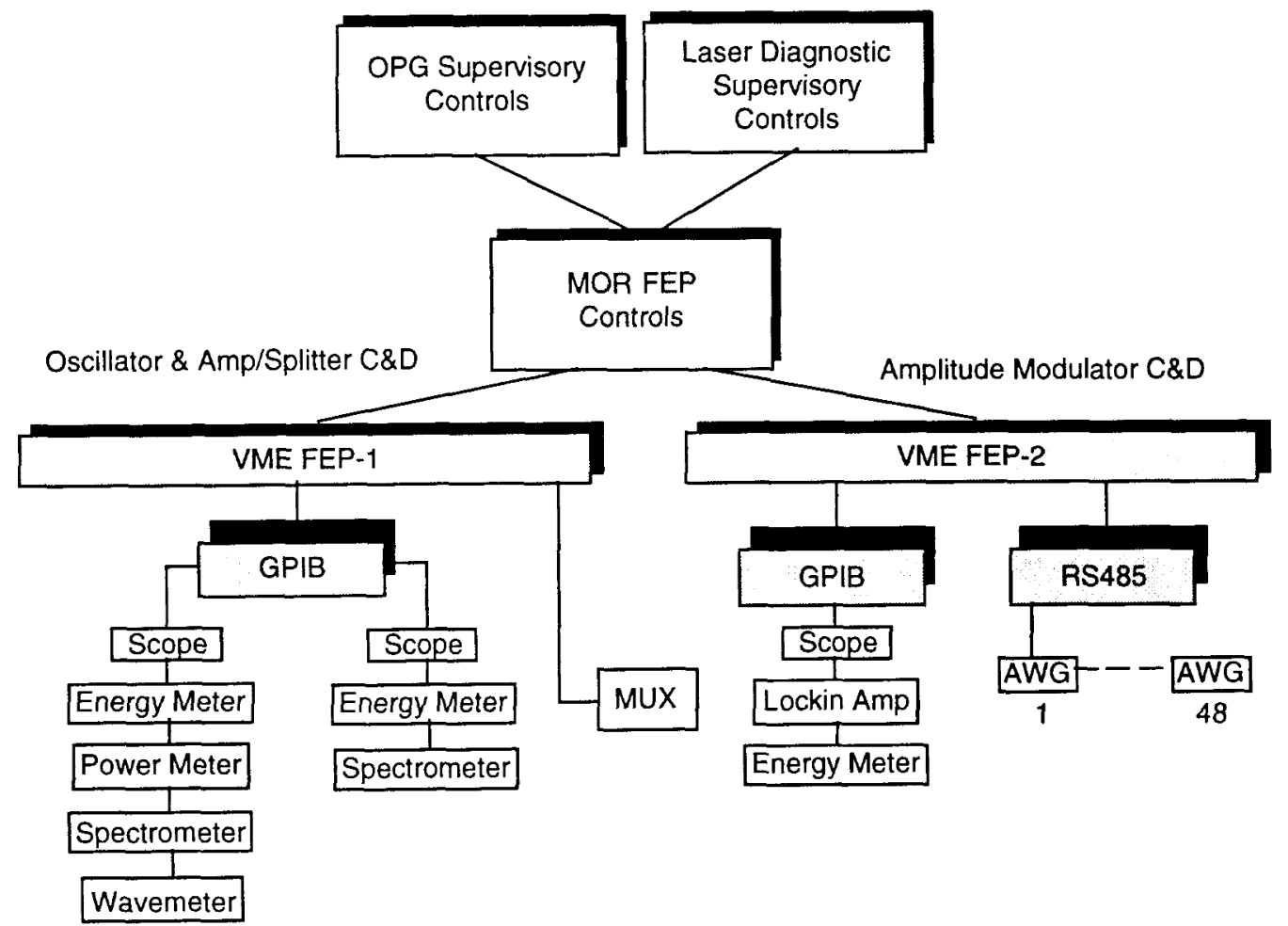

Figure 17 Optical pulse generator FEP architecture

Two FEPs are installed in the MOR. One FEP is responsible for the acquisition of diagnostic data used to characterize the master oscillator pulses, while the second FEP is responsible for control of each of the 48 optical amplitude modulators.

Through the use of arbitrary waveform generators, the amplitude modulators allow the generation of shaped temporal waveforms of up to 21 nanoseconds duration. Laser energy and power balance as well as pulse timing is achieved through the ability to vary the laser pulse amplitude as a function of time.

Key diagnostic control points are the ring oscillator diagnostics, the amplifier/splitter diagnostics, and the amplitude modulator diagnostics. On the control side, the setup of each of the 48 arbitrary waveform generators (AWG) are key control points. The AWG is a nonlinear device that requires an iterative process to generate a specified arbitrary output pulse.

The oscillator diagnostics consist of an energy meter, a power meter, a fast sampling oscilloscope, a Fizeau-type wavemeter and a scanning Fabry-Perot spectrometer. Each of these devices are standard GPIB interfaced instrumentation. Additionally, the $30 \mathrm{~ns}$ pulse that is chopped out of the master oscillator pulse can be 
approximately shaped in amplitude to compensate for gain distortion in the optical amplifier/splitter section. This is handled with a programmable waveform generator. The FEP controls these GPIB devices and provides the diagnostic data to the OPG supervisor.

The diagnostics for the amplifier/splitter section consist of a spectrometer, an energy meter, and an oscilloscope. These diagnostics are primarily dedicated to ensuring that there is no ASE or spurious oscillations on the amplifier/splitters. Only one set of diagnostics is used, and the input is optically multiplexed to each of the 48 amplifier outputs. The FEP controls this multiplexer, and acquires diagnostic data from the instruments.

The amplitude modulator diagnostics consist of an energy meter and a fast sampling oscilloscope that measures the optical pulse of each of the 48 amplitude modulators. Again, there is only one set of diagnostics, and the diagnostic inputs are optically multiplexed. The electro-optical modulator device requires an optimal pulsed bias voltage applied to it's terminals. The value of this bias (which has to do with the extinction of the output pulse with no modulation input) is determined through the use of a lock-in amplifier to minimize the noise associated with the low-level output. The FEP controls these modulator diagnostics, and handles the optimization of the bias voltage. One FEP controls all these diagnostics.

The second FEP handles the setup of the AWG. Each AWG utilizes a 96 point array of unsigned 16-bit integers used to establish bias voltages to an array of 96 field effect transistors (FET) distributed along a tapped transmission line. Each FET can be thought of as a variable load separated in time along the transmission line by $250 \mathrm{ps}$. By varying the bias voltage to a FET, an impulse of varying amplitude is generated. The output pulse is a summation of the 96 impulses. Unfortunately, the amplitude of the output impulse is a nonlinear function of the bias value, and the optimal set of FET bias values to generate a specified waveform is an iterative solution process. A fast sampling oscilloscope monitors the output optical pulse from the amplitude modulator, and the bias voltages are adjusted until the specified output pulse is achieved. The FEP accepts a specified waveform from the supervisory level, and handles the generation of the output pulse to a specified tolerance. One amplitude modulator is done at a time until all 48 modulators are setup. This is a process that may last approximately one hour for all the modulators.

The diagnostics used in the MOR are standard GPIB programmable devices. The AWG contains an embedded controller, and the FEP communicates to the AWG through a multi-drop RS485 serial interface.

Time of convergence to a specified waveform is a critical. A critical failure mode is the absence of the SBS suppression modulation. If this modulation is not present, the output from the MOR is inhibited. This interlock is handled in hardware. 
As noted above, there is a hardware fail-safe mechanism on the SBS suppression device. The FEP will read and report the status of this device, but it is not responsible for inhibiting the pulse. The SBS fail-safe is a Q Level 1 system. A Q Level 2 failure of the amplitude modulators that results in too high of an amplitude pulse, or a pulse that can damage down-stream laser components is handled through a separate PAM fail-safe mechanism.

\subsection{Preamplifier Module (PAM) FEP}

The NIF Optical Pulse Generation (OPG) system [Figure 18] consists of 48 Line Replaceable Unit (LRU) Pre-Amplifier Modules (PAMs) which serve to amplify the master oscillator pulse of a one nanoJoule level to a level suitable to drive a quad of the main laser amplifiers. The PAM consists of two cascade laser amplifiers. The first amplifier section is a solid state diode pumped regenerative laser amplifier which boosts the seed pulse (delivered to the PAM on polarization maintaining fiber from the MOR) to a $10 \mathrm{~mJ}$ level at the output of the regenerative amplifier on a 1 pulse per second basis.

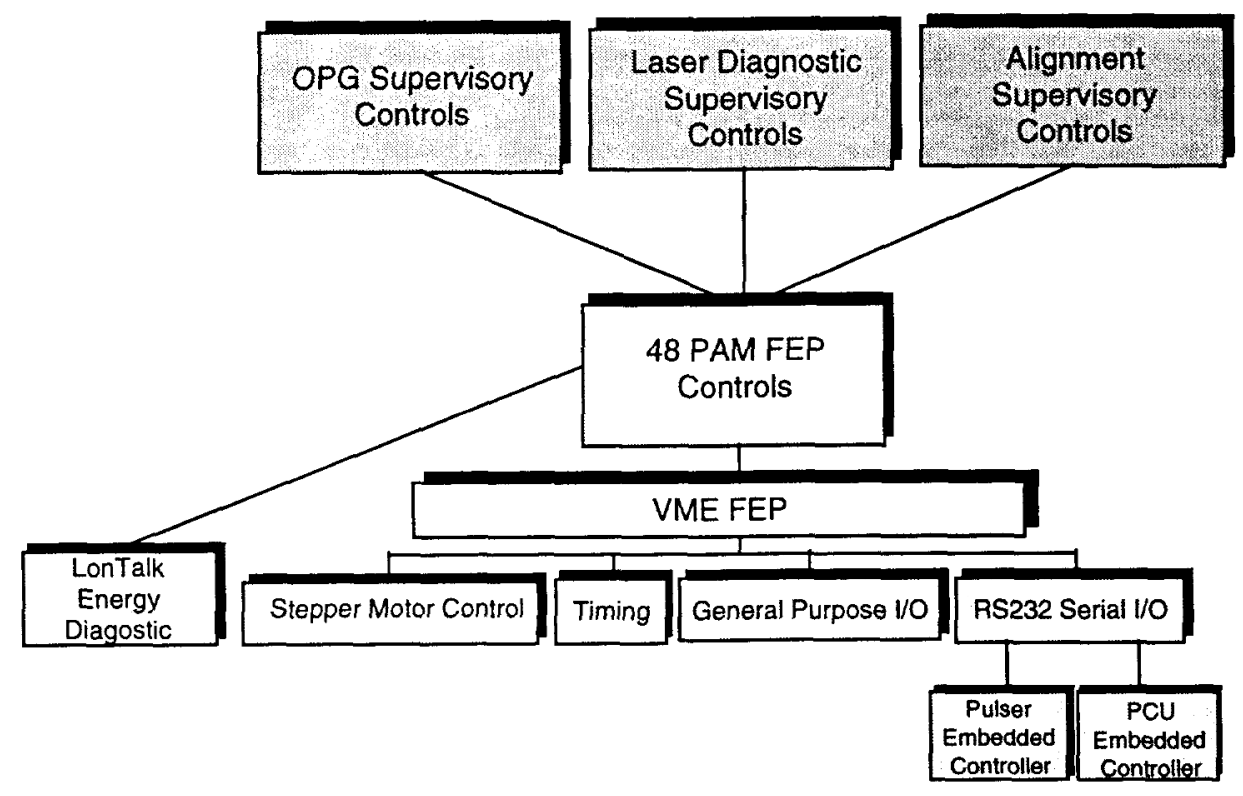

\section{Figure 18 Preamplifier module FEP architecture}

Between the first amplifier section and the second amplifier is a beam-shaping module that transforms the circular shaped beam into a square shaped beam. The second amplifier stage is a four-pass flashlamp pumped rod amplifier. Here the light is amplified to a maximum energy of 22 Joules, with a shot every 20 minutes.

Optical alignment of the laser chain originates in the PAM, and several of the optical elements are cam be remotely aligned through the PAM FEP. The PAM FEP also 
provides control of the PAM hardware to achieve energy balance and proper operation.

Key control points from the supervisory system are the set points of the diode pulser and waveplate settings for the regenerative amplifier, and the operation of the PCU for the multi-pass amplifier (MPA). Setup of the embedded timing module is necessary to synchronize the PAM pulse with the rest of NIF, and a total of sixteen motorized devices are controllable for laser alignment. Control of these devices is shared between OPG, Laser Diagnostics, Alignment and Timing.

Each PAM contains an embedded VME-based FEP to remotely control the operation of the amplifier. These control functions include control over the electrical pulser that drives a diode pumped laser, control of motorized devices such as rotary waveplates to adjust amplifier gain and motorized gimbaled mirror assemblies for laser beam pointing and centering alignment. Additionally, the FEP controls an associated $40 \mathrm{~kJ}$ capacitor bank (power conditioning unit or PCU) which drives the flashlamp pumped rod amplifier, and allows remote selection of 0-D or 1-D SSD operation as well as the insertion of various alignment devices and references. Finally, the FEP maintains the integrity of the PAM operation through the control of internal ion vacuum pumps, thermal control loops, electrical power control and internal interlocks, and system timing.

The PAM FEP control system distributes control to the lowest level possible. Within the PAM, there are embedded controllers for both the PCU and the diode pulser. Each of these embedded controllers communicates with the PAM FEP via RS232 serial links. The embedded controllers help to ensure fail-safe operation of their respective devices, for example controller in the diode pulser verifies set points so that the hardware cannot be damaged by out of range operation. Similarly, the PCU embedded controller handles the proper sequence of charge/discharge operation required for safe operation. Hardware devices within the PAM, such as the stepper motors used by the Alignment Supervisors, are controlled through the internal PAM FEP, not a separate Alignment FEP outside the PAM enclosure.

The OPG utilizes the LONworks network ( as used by Laser Diagnostics) to control and acquire data from an input energy diagnostic within the PAM.

Total energy and peak power outputs from the PAM are Q-Level 2 key constraints for it's operation. PAM operation out of limit can cause damage to the main laser amplifiers. There are also constraints on the operation of the PCU in terms of high voltage and personnel safety, and this unit is interlocked through the Integrated Safety System.

\subsection{PEPC FEP}

The Plasma Electrode Pockels Cell (PEPC) together with the Polarizer function as an optical switch in the Main Amplifier Cavity to allow for multipass amplification [Figure 19]. A Pockels cell is an electro-optic device that rotates the polarization of 
light by applying a voltage to a crystal to induce birefringence. For a particular value of applied voltage, the polarization of the beam is rotated exactly $90^{\circ}$. This aligns the light polarization with the polarizer, allowing the beam to pass through and remain in the cavity.

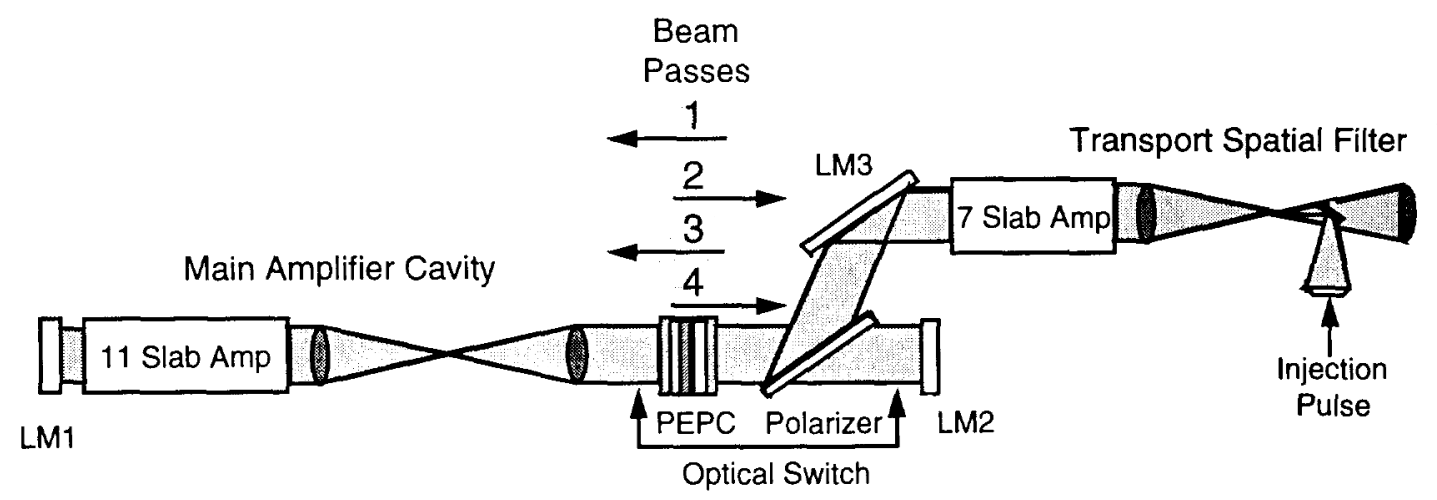

Figure 19 PEPC and polarizer location in the NIF beamline

The beam is first injected in the Transport Spatial Filter. After it enters the Main Amplifier Cavity, the beam makes 4 passes through the PEPC. During the first pass the PEPC switch voltage is off and the beam is not rotated. During the second and third passes, the PEPC switch voltage is on and beam is rotated $90^{\circ}$ for pass 2 and another $90^{\circ}$ for pass 3 (a total of $180^{\circ}$ ). While the beam is at $90^{\circ}$ rotation, it passes through the polarizer, reflects off the LM2 mirror, and back through the polarizer. On the fourth pass through the PEPC, the switch voltage is off and the unrotated beam reflects off the polarizer to exit the Main Amplifier Cavity.

To accommodate the large beam size of the NIF, the PEPC uses plasma electrodes to conduct the switch voltage across the face of KDP crystals. Figure 20 shows a crosssection of a PEPC and a simplified schematic of the external electronic circuit. Vacuum regions on each side of the crystal are filled with working gas at an optimized pressure. The plasma pulsers ionize this gas with a high-current pulse, forming the plasma-electrodes. Voltage from the switch pulser is applied across the crystal via these plasma-electrodes. 


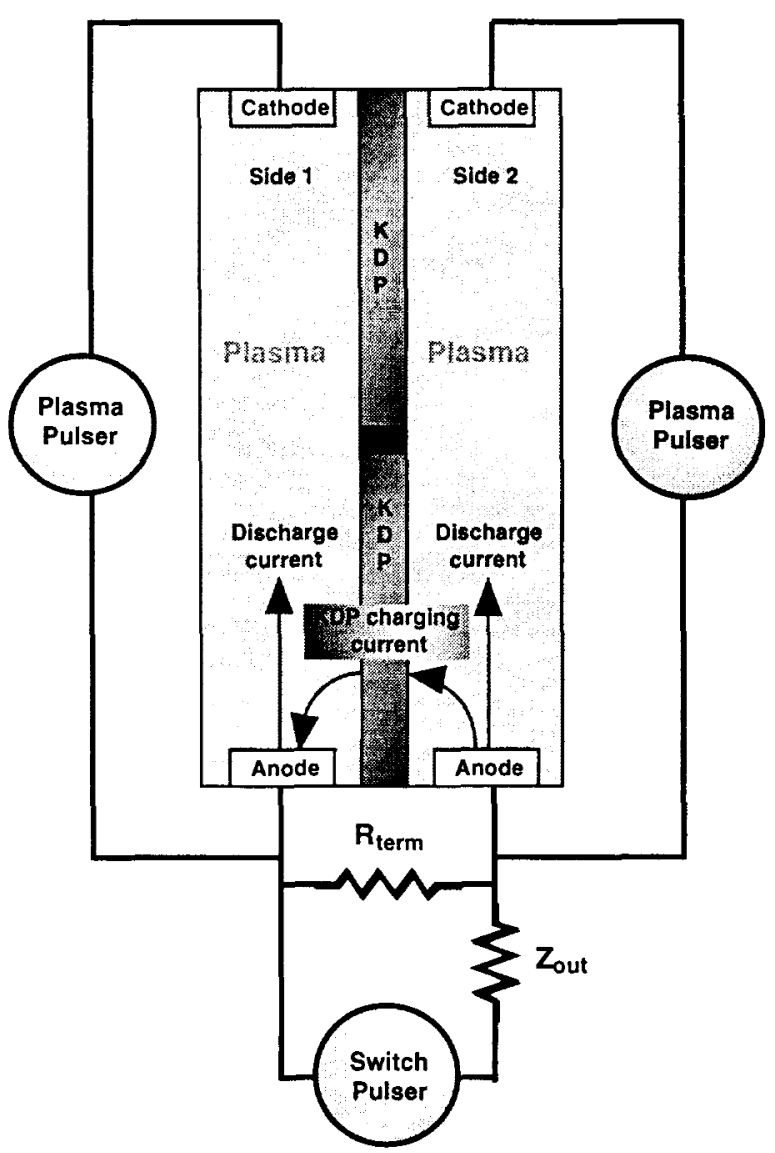

Figure 20 Plasma Electrode Pockels Cell schematic

Front-end controls provide sequenced control and monitoring of the vacuum, gas, plasma currents, and switch pulse of each PEPC Unit. In NIF, there will be three PEPC FEPs per Cluster [Figure 21]. One FEP will control and monitor the switch pulsers; another FEP will control and monitor the plasma pulsers and gas and vacuum. The third FEP is responsible for the acquisition of the high-speed switch pulses and plasma pulses to verify correct operation.

The PEPC FEPs control the Plasma Pulse Generators, Switch Pulse Generators, Turbomolecular Pump Embedded Controllers, Gas and Gauge Embedded Controllers, and Gas \& Vacuum Valves. The PEPC FEPs monitor the switch and plasma pulses; the output voltage and current of the various power supplies in the Pulse Generators, gas and vacuum pressures of the plasma regions and the vacuum foreline, gas bottle pressure, and safety interlock states. 


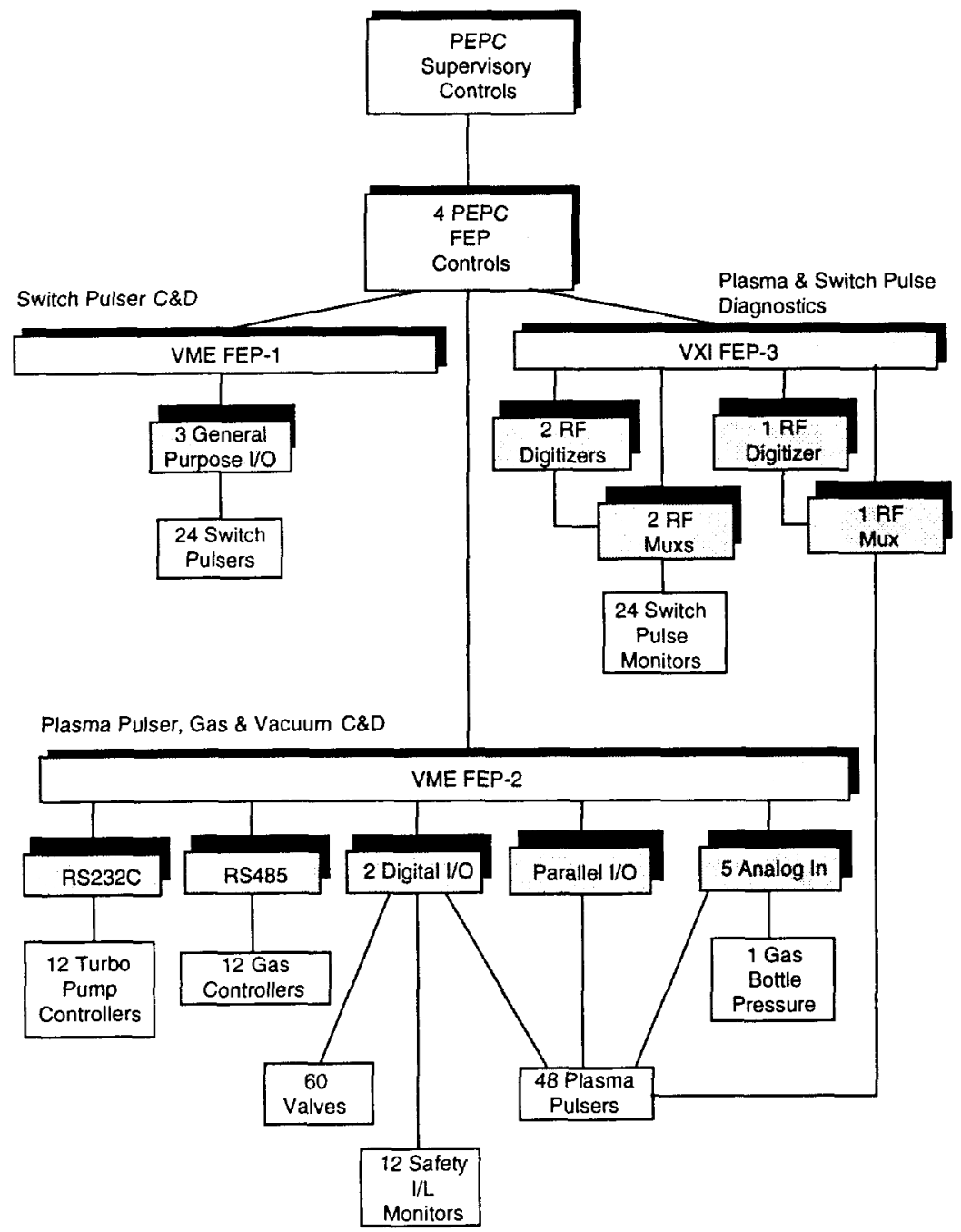

Figure 21 Plasma Electrode Pockels Cell FEP architecture

The vacuum and gas system provides the required environment inside the PEPC for optimum formation of the plasma electrodes. A turbomolecular pump evacuates the PEPC interior to less than $5 * 10^{-5}$ Torr to ensure that the concentration of impurity species in the plasma is low enough that it does not degrade the discharge uniformity. The gas system injects the working gas (a mixture of $\mathrm{He}$ plus $1 \% \mathrm{O}_{2}$ ) into the cell and maintains the gas pressure at $35 \mathrm{mT}$ Trr with active feedback control.

The plasma is created in a two-stage process: 1) a low-current preionization (simmer) discharge and 2) a high current pulsed discharge. The simmer discharge is initiated by a high-voltage, low current power supply that provides enough voltage to break down the gas and thereafter maintains a constant discharge current. Discharging stored energy from a capacitor produces the high-current pulse. 
Key control points are the switch pulse timing and switch voltage level. The switch voltage pulse timing must be synchronized with the multipass beam in the Main Amplifier Cavity so that the voltage is off during passes 1 and 4 , and on during passes 2 and 3. The switch pulse is verified by comparing the sampled pulse to a reference pulse, and by aligning the pulse timing via fiducials supplied by the Integrated Timing System. Closed loop control to within 1 nanosecond is performed on the switch trigger timing.

The gas and gauge embedded controller is used to provide active feedback control of the gas flow in the plasma regions. It uses a capacitance manometer to monitor the cell pressure and drives a servo loop that actuates a gas-metering valve. The gas and gauge controller is also used to control all the gauges on the PEPC and the vacuum foreline. The FEP communicates to this controller via serial link to set pressure setpoints and feedback control loop parameters, turn the gas on and off, and monitor and control the gauges.

The turbo pump controller operates the two-stage turbomolecular pump that continuously evacuates the plasma regions. The FEP communicates to this controller via serial link to turn it on and off, and to monitor fault conditions.

\subsection{Power Conditioning FEP}

Four Power Conditioning FEP control 192 Bank Modules [Figure 22]. Each Bank Module has a main capacitor bank which stores $\sim 1.6$ megaJoules (MJ) and drives 40 flashlamp pairs used in the main and power amplifiers at NIF and also has a Preionization and Lamp Check (PILC) capacitor bank. The Bank Modules are located in 4 capacitor bays with one FEP located in each bay. Each FEP controls 48 embedded controllers. 


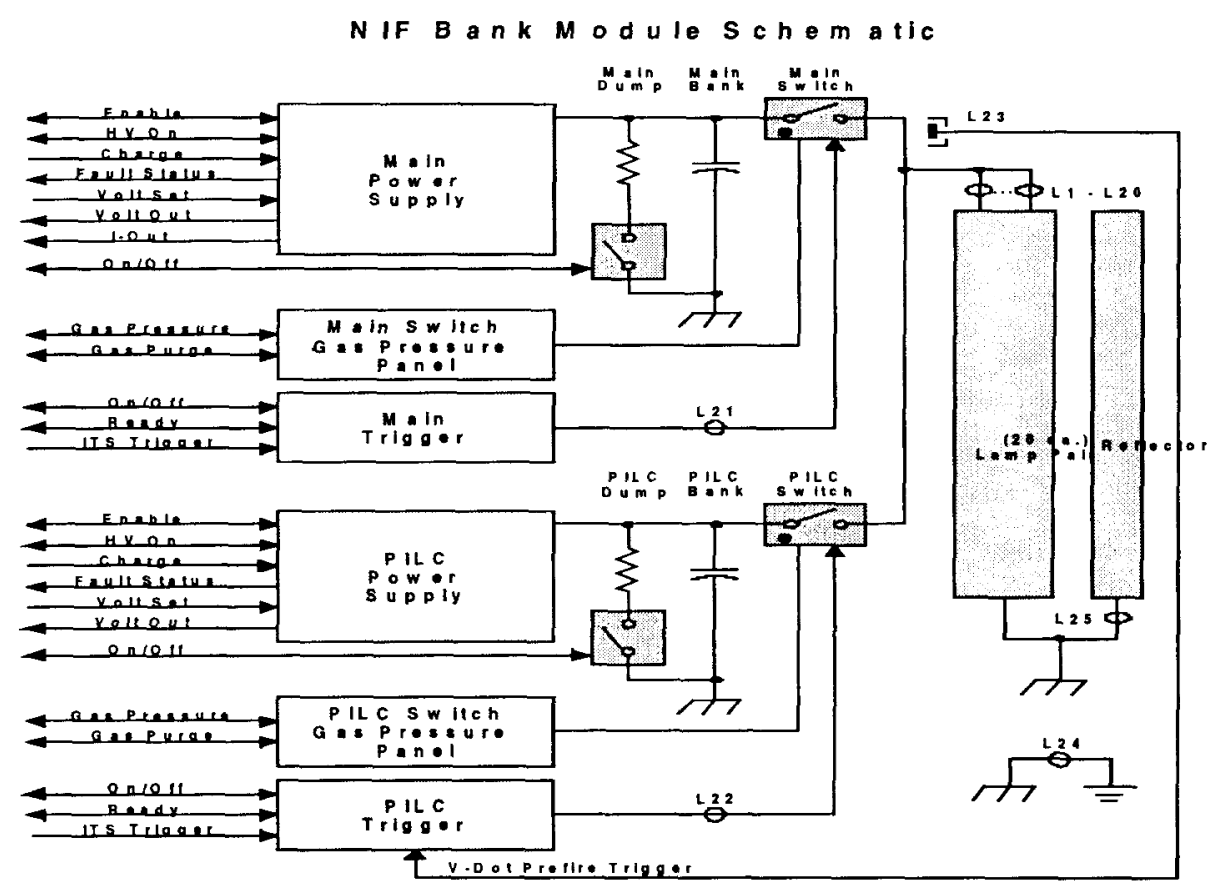

\section{Figure 22 Power Conditioning Bank Module Schematic}

Each Power Conditioning FEP provides a CORBA interface with the ICCS for 48 Bank Modules. In addition, the message framework, the status framework, the archive framework, etc. are implemented in the FEP. The FEP is proposed to be a Sun Ultra-Server 1 System using the Solaris operating system. The FEPs will be rack mounted and installed in the capacitor bays.

Communication between the FEPs and the Integrated Computer Controls System (ICCS) will be via a $10 \mathrm{Mbit} / \mathrm{s}$ Ethernet computer network using CORBA communication. Communication between the FEP and the embedded controllers will be over the same network using TCP/IP communication.

There is one embedded controller in each Bank Module. The key control points for each embedded controller are 2 high voltage power supplies, 3 dump switches, 2 gas panels, 2 trigger systems, and 25 current monitors. In total, the Power Conditioning embedded controllers control 384 high voltage power supplies, 576 dump switches, 384 gas panels, 384 trigger systems, and 4,800 current monitors which are distributed throughout the four capacitor bays.

Each embedded controller sets power supply output voltages, monitors main and PILC output bank charge voltage and main bank charge current, monitors gas pressure in switches, purges gas switches, arms trigger circuits, dumps energy stored in capacitor banks, and records transient current data. 
Each embedded controller is proposed to be an Intel X86-class computer. The operating system is VxWorks and the language used will be either $\mathrm{C}++$ or Ada. A STD-32 bus is used in the Bank Module. Two digital I/O interface boards provide digital I/O for the power supplies, dump switches, etc., and one analog board provides the analog $\mathrm{I} / \mathrm{O}$ required by the power supplies. The embedded controller will be installed within an EMI shielded enclosure which itself will be installed in a Bank Module control rack.

Communication between the FEP and the embedded controllers will be over a 10 $\mathrm{Mbit} / \mathrm{s}$ Ethernet computer network using TCP/IP sockets communication. The interface between an embedded controller and the Bank Module is under development.

\subsection{Timing FEP}

The Integrated Timing System (ITS) provides over 2000 precisely timed signals or timing markers (fiducials) to devices and other systems (clients) activities. All timing signals are synchronized to a single Master Clock source.

During shots or dry runs, these signals occur at programmed times from one second before to one second after the laser pulse. At other times, the ITS supports activities such as maintenance and calibration by providing triggers at several repetition rates and also client-specified single shot triggers.

Three classes of trigger performance are supported by the ITS: Extended Range, Fast and Precision classes. The performance parameters of the trigger classes are shown in [Table 4].

\begin{tabular}{|l|l|l|l|}
\hline \multicolumn{1}{|c|}{ Trigger class } & \multicolumn{1}{c|}{ Range } & \multicolumn{1}{c|}{ Resolution } & \multicolumn{1}{c|}{ Stability } \\
\hline Extended & \pm 1 second range & $<100 \mathrm{~ns}$ & $<100 \mathrm{~ns}$ \\
\hline Fast & $\pm 1 \mathrm{~ms}$ & $<150 \mathrm{ps}$ & $<150 \mathrm{ps}$ \\
\hline Precision & $\pm 10 \mu \mathrm{s}$ & $<30 \mathrm{ps}$ & $<30 \mathrm{ps}$ \\
\hline
\end{tabular}

Table 4 Timing System Trigger Performance

The relative timing of triggers is determined by programmable delay generators, which are controlled by the ITS in response to requests from clients. The physical length of electrical and optical cables sets fiducial timing.

The ITS is divided into three major subsystems:

- Facility Master Timing maintains replicas of the Master Clock in each of 11 zones throughout the NIF.

- Local Timing Distribution uses the replica clocks and, after a programmed delay, generates triggers that are transmitted to clients' equipment. 
- Cross-Timing regenerates a fiducial signal originating in the Master Oscillator Room for use by clients to synchronize recorded information from diagnostic instrumentation with the laser beams arriving at the target.

The FEPs supporting these subsystems interface the ITS to its clients and the various databases that contribute to determining the actual delay values programmed into the delay generators.

\subsubsection{Timing Master FEP}

There are two types of FEPs supporting the Integrated Timing System; a single Master FEP located in the NIF Master Oscillator Room and multiple Zone FEPs distributed near client equipment throughout the Facility [Figure 23 and Figure 24].

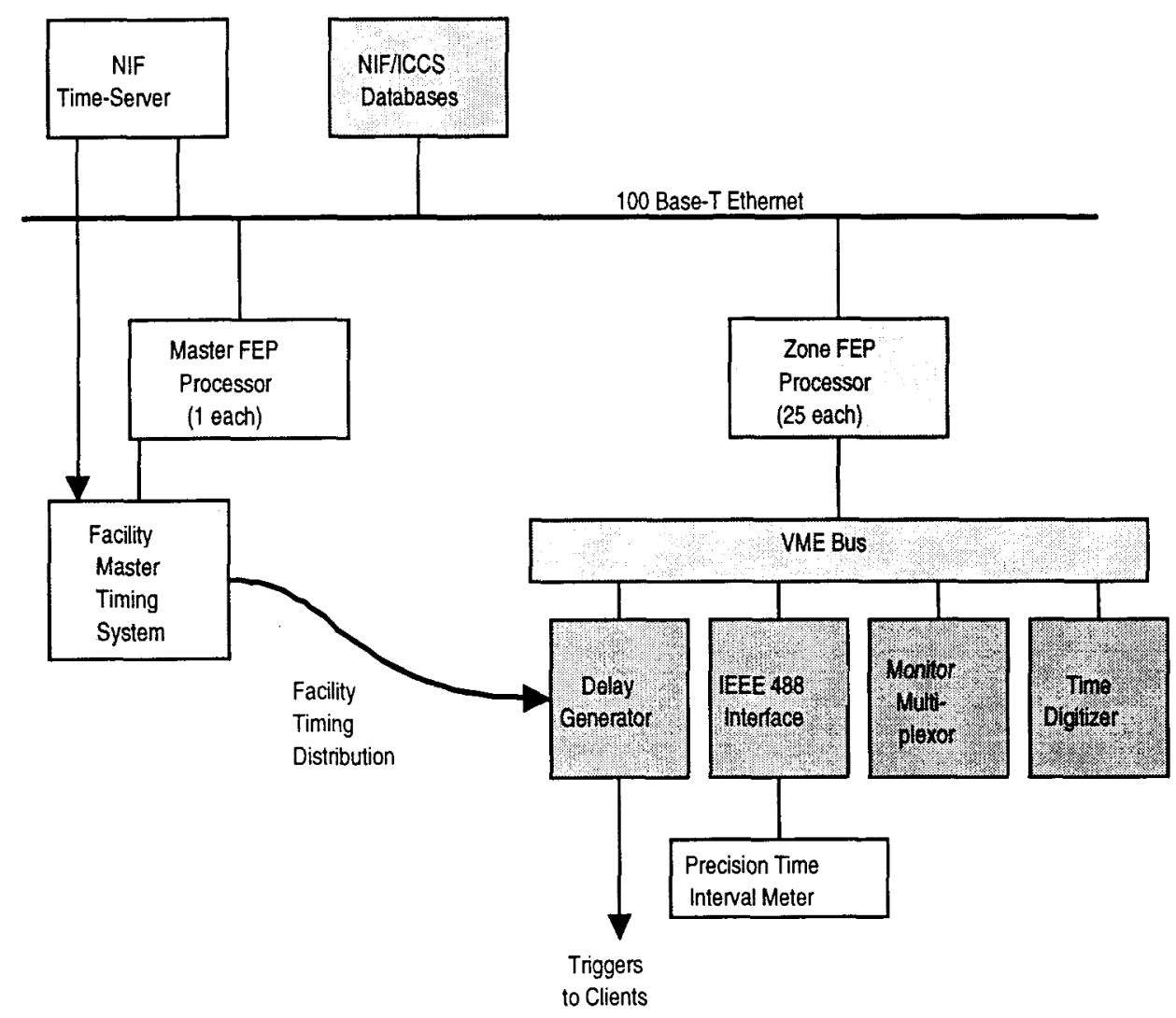

Figure 23 Timing System Architecture 


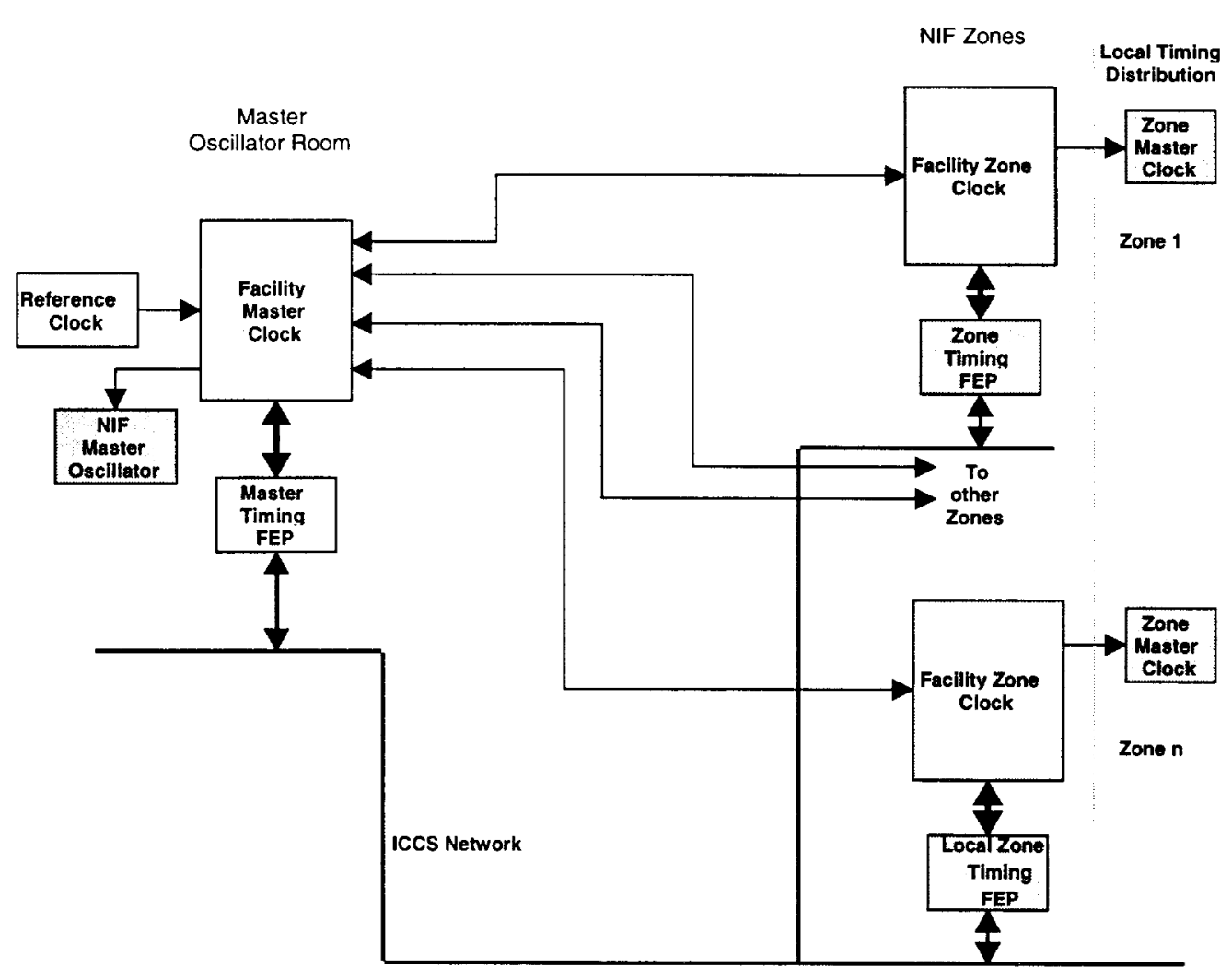

Figure 24 Facility Timing Distribution Subsystem Diagram

A Facility Master Clock (FMC) synchronizes the NIF Master Oscillator with the ITS through a $960-\mathrm{Hz}$ clock generated by the FMC. The Master Oscillator is triggered by the $960-\mathrm{Hz}$ clock, and the delay to the optical outputs of the Master Oscillator are held constant to $<10 \mathrm{ps}$ in order to satisfy the requirements of Precision Timing.

The Master FEP interfaces to the Facility Master Timing System. It provides an accurate stable reference clock along with low-frequency epoch clocks and triggers to each of the local timing zones. A Two-Way Time Transfer technique is used to actively monitor and compensate for time delays between the Facility Master Clock and the Facility Zone Clocks.

In addition to supporting Two-Way Time Transfer synchronization, the data stream that flows from the Facility Master Clock to the Facility Zone Clocks also carries encoded triggers for various repetition rates and single-shot events. This data stream is passed from the Facility Timing to the Local Timing Distribution system in each zone and ultimately to delay generators that produce triggers.

Only those delay generator channels that are preprogrammed by their Zone FEP with the transmitted keys will trigger when the key is activated. The Master Timing FEP will also be responsible for reserving and canceling these keys for clients. Since 
the Master Timing FEP is the single source of generating trigger keys for the NIF, a redundant system will be available in the MOR zone for rapid switch over.

Zone FEPs (Section 9.5.2) control the equipment that generates and verifies triggers that are delivered to clients' equipment.

Clients make timing requests that are relative to their time frame of reference. The ITS accepts these requests and makes adjustments based on information maintained in several data bases to transform the client reference frame to the ITS reference frame. The Zone FEP then sends a command to a programmable delay generator channel that results in the trigger signal being generated at the specified time relative to a NIF shot.

The Master Timing FEP also monitors the overall status of the ITS, including the status of the Local Zone Timing FEPs, the Facility Timing Distribution subsystem and the Cross Timing subsystem. It will also serve as the single ITS interface to the NIF ICCS Shot Director.

The Master FEP consists of a 21-slot VME crate with a PowerPC processor running the VxWorks operating system. It interfaces to the subsystems and ICCS via 100 Base-T Ethernet. Since it also serves as a Local Timing FEP within the MOR timing zone, it contains delay generators for generating the triggers and time interval meters for performing trigger diagnostics. Timing data will also be distributed over the Facility Timing Distribution subsystem to delay generators within each zone.

Timing keys generated and distributed by the Facility Timing Distribution subsystem are inhibited from being broadcast to delay generators if the T-1 abort signal from the Industrial Controls subsystem is not present. In effect, this prevents the shot from occurring within the last second of shot $t_{0}$.

\subsubsection{Timing Zone FEP}

Local Timing FEPs are located in the Local Timing Zones. There are 25 Local Timing FEPs for NIF. Each FEP receives requests from clients to set trigger channels that correspond to delay generator channels. Delays requested by clients are referenced to the client's timing frame of reference. The Local FEP accesses information maintained in several ICCS and ITS databases in order to transform the client reference frame to the ITS reference frame. The Local FEP programs a delay generator channel that results in the trigger signal being generated as the client requested and at specified times relative to a NIF shot. Clients can also enable or disable a trigger channel and read a trigger channel's settings. The Local Timing FEP monitors the status of delay generators via time interval meters.

The regenerated clocks, which are provided by each Zone Master Clock, are distributed within the zone and several clients may be served in a given zone. Each client subsystem has full control of the parameters associated with every delay channel trigger output that it has reserved. In order to assure the integrity of the 
triggers, there is extensive monitoring of the facility-wide distribution and selective monitoring of the local area distribution. This monitoring is intended to bring timing system problems to the attention of an operator before they are manifested on a NIF system shot. The monitoring capability is also essential to maintaining the performance of the Local Timing Subsystem in the event of hardware replacement due to routine maintenance or component failure.

Each Local Timing FEP consists of a 21-slot VME crate with a PowerPC running the VxWorks operating system. It interfaces to the subsystems and the ICCS via 100 Base-T Ethernet. It contains 6- or 8-channel Delay Generator VME modules (up to 16 in each VME crate) and Time-to-Digital Converters (for Extended Range and Fast trigger channels). It also contains a GPIB interface to a high-resolution Time Interval Counter yielding 25 ps resolution. These time interval devices will be used to diagnose the status and performance of the delay generators.

\subsection{Automatic Alignment FEP}

The NIF is actively aligned by adjusting motorized components at many locations along the beamline. Alignment activities include pointing, centering, orienting, and focusing. Over 4,000 devices are manipulated during the alignment process including mirrors, lenses, polarizers, shutters, light sources, reticles, and attenuators. Several computer systems are involved in alignment operations: Automatic Alignment FEP, Wavefront FEP, Video FEP, and the Alignment Supervisory [Figure 25].

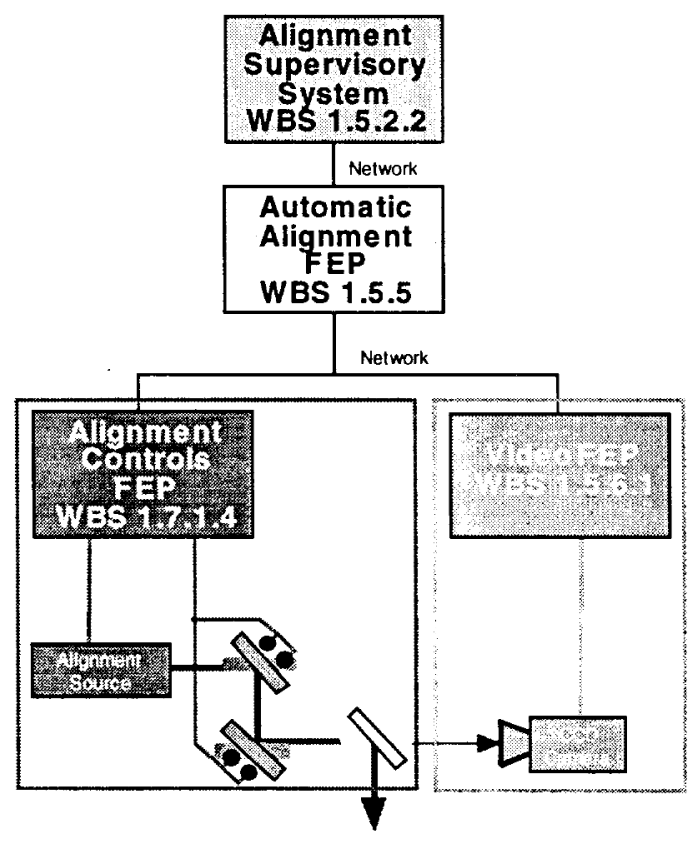

Figure 25 Automatic alignment FEP architecture 
The primary function of the Automatic Alignment Front End Processor (AAFEP) is to automatically align the 192 beamlines to the required tolerances. The system determines alignment error through analysis of video images (supplied by the Video FEP) and corrects the error by manipulating optical devices (controlled by the Alignment Controls FEP). To accomplish alignment in the required 1 hour time period, all beamlines, as well as multiple segments of each beamline must be aligned concurrently.

The primary functions required for automatic alignment are:

- Perform pointing, centering, orienting, and focusing

- Provide concurrent operations to align beamlines and segments in parallel

- Provide control loop operations to analyze video images, determine error and adjust alignment devices

- Provide interface to manual alignment

- Interface and control classified / unclassified target area hardware

- Perform electro-optical characterization of all alignment devices

- Archive imagery and alignment data

- Perform beam search algorithms for all systems

- Verify device functionality for all systems

The supervisory system indicates when alignment is to begin and provides initialization to the AAFEP. All images required by the AAFEP are provided by the Video FEP. The Alignment Controls FEP adjusts devices (such as shutters, light sources, mirrors, and lenses) in response to AAFEP requests. The AAFEP does not directly control video or devices, but rather issues explicit network commands to remotely control these devices through these two other FEPs.

The alignment control loop operations include:

- Setup beam path - The beam path must be setup to allow the proper light source to be viewed by the required sensor.

- Acquire reference image - Images are acquired from the Video FEP

- Setup beam path

- Acquire alignment image

- Determine alignment error by analyzing imagery and comparing image features

- Adjust alignment optics

- Additional alignment images are analyzed and devices adjusted until the alignment error is below the required specifications

The system requires a high-speed computer to perform the image processing algorithms that analyze imagery from each of the 192 beams, often concurrently. General computer systems are now available that will meet the processing requirements and provide a better system design than specialized, non-standard image processing systems. 
A Sun Microsystems server (Enterprise 3000 server) with its multiprocessor capability and high-speed backplane was selected because of its scaleable processing flexibility. If image processing demands are higher than expected, additional CPU's can be added to the system, without effecting the hardware or software designs.

Although alignment systems have been built and demonstrated, and image simulations have been created based on the current beamline design, it is difficult to predict precisely what image processing algorithms will be required and what control loop sequence will operate most effectively. For these reasons, flexibility was built into the system to allow algorithms to be swapped and sequencing logic to be changed without rewriting the software. Scripts will be utilized whenever dynamic operations are required. Scripts are easily rewritten and updated during the system activation and maintenance.

\subsection{Video FEP}

The Video FEP performs monitoring of cameras distributed throughout the laser beam lines and target area [Figure 26]. There are about 600 video cameras distributed throughout the laser beamlines, including 192 cameras used for wavefront control. The FEP monitors video cameras throughout the facility, uses frame grabbers to digitize the video signals, and transmits it over the network to the automatic alignment system. The FEP also provides motion-video display at operator consoles. Capability for displaying camera identity and fiducial overlay on displayed images is provided.

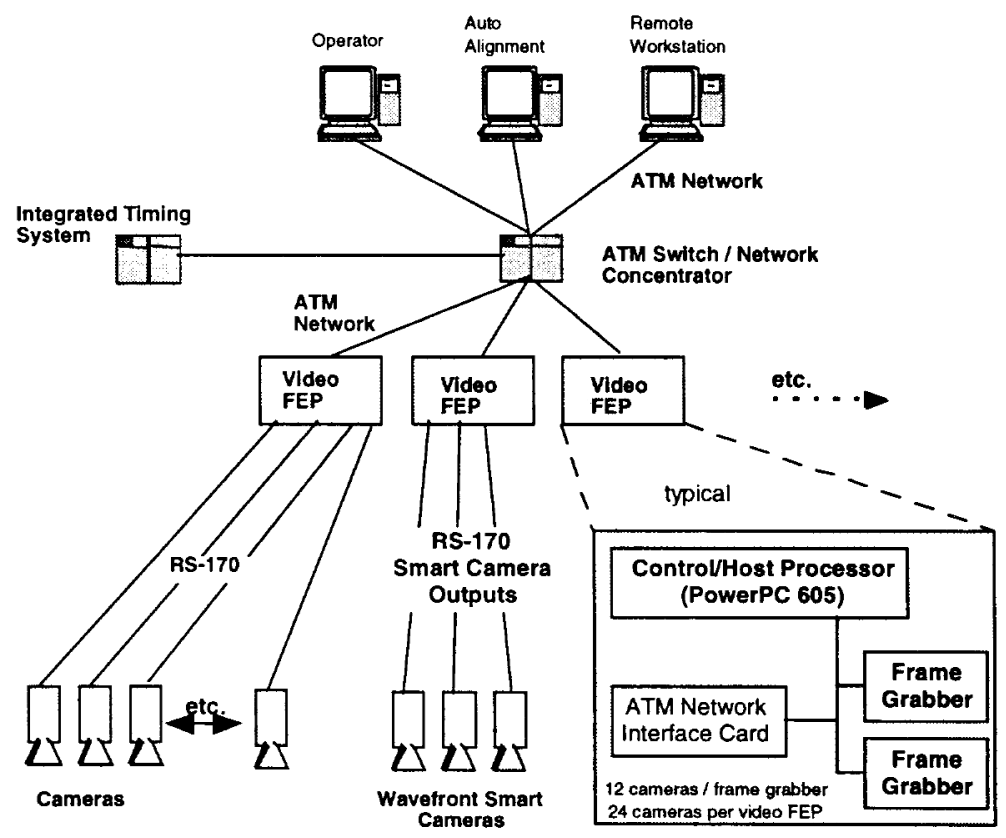

Figure 26 Digital video FEP architecture 


\subsection{Industrial Controls FEP}

The Industrial Controls System (ICS) Front End Processor (FEP) interfaces twelve separate Industrial Controls application areas to the Supervisory System [Figure 27]. Programmable Logic Controllers (PLC), and a Personal Computer in the case of the Access Control System, individually control these application areas. The twelve application areas are described below.

\section{Industrial Controls Systems}

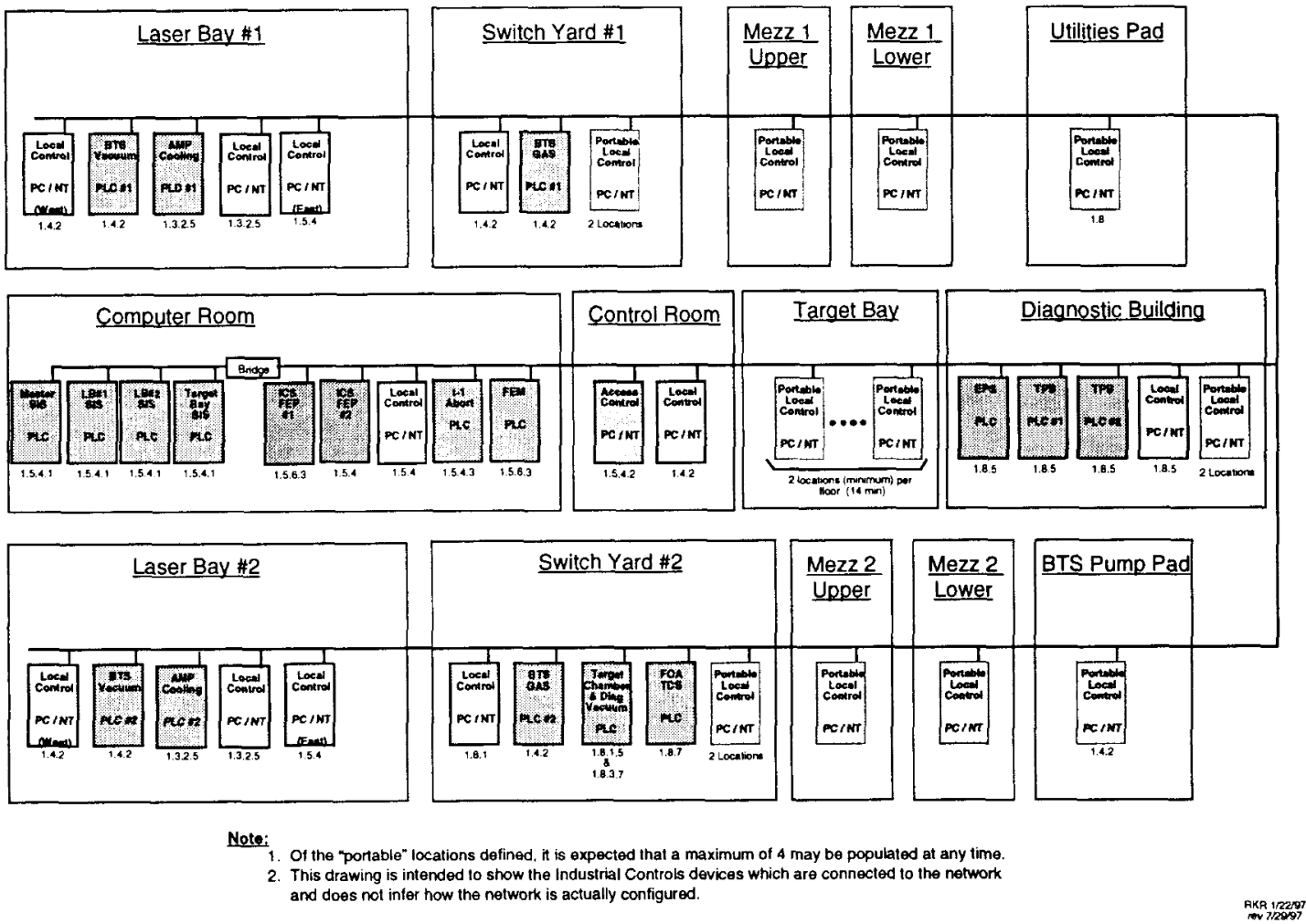

Figure 27 Industrial control system architecture

The individual PLC or control processors (controller) execute control in each of the twelve application areas of the Industrial Controls FEP. In certain cases the ICS FEP sends command request to the controlled system, however the controller executes the control and has the ability to deny the request if it is not permissible to execute the request.

The ICS FEP concentrates data from the twelve separate application area controllers, translates the data into a format required by the Supervisory System, and sends the data the ICS Supervisor via CORBA. Commands, acknowledgments, and configuration information sent by the ICS Supervisor are translated by the ICS FEP into the format required by the controllers. Each controller is capable of stand-alone operation with the limitation of permissive and configuration information that is 
required in some areas. The FEP is also capable of stand-alone operation and has a local disk for archiving. The FEP monitors data and alarms in the controller and sends data to the ICS Supervisor when a significant change is registered.

The PLCs in each area will communicate to each other (where multiple PLCs or multiple input/output ( $/ / O)$ drops are used) using a commercial local network supplied and supported by the vendor. Communication with the ICS FEP will be accomplished using standard 10baseT Ethernet.

Because the ICS operates around the clock, the system is being designed, where possible, to operate in a stand-alone mode where interaction with the ICCS supervisory software is allowed but not required. This requirement defines that the ICS FEP must have the capability to run stand-alone as well, and must be capable of data archiving in case the ICCS system is unavailable so that continuous data archives are maintained.

Subsystems controlled and monitored by the ICS are discussed below.

\subsubsection{Amplifier Cooling System}

The Amplifier Cooling System flows nitrogen gas into the flashlamp cavities of the Main Amplifier and Power Amplifier of the NIF laser to remove heat from the flashlamps after the laser shot. Heat is removed to prevent thermal migration to the amplifier slabs, which can causes long-term distortion in the laser beam quality. This system carefully controls the temperature and humidity of the gas as the beams are being cooled.

\subsubsection{Beam Transport Vacuum System}

The Beam Transport System Vacuum Control System (BTSVCS) evacuates and maintains a vacuum of $10^{-4}$ Torr in the Transport and Cavity Spatial Filters. This system is a multi-stage vacuum system with roughing pumps, turbo pumps, cryo pumps, and a variety of instrumentation used to measure the pressure ranging from atmosphere to high vacuum.

\subsubsection{Beam Transport Gas System}

The Beam Transport System Gas Control System (BTS GCS) purges the air and maintains an atmosphere of argon gas inside the beam tubes located in the switchyard between the TSF and Final Optics Assembly. Purging of the air in the beam tubes and back filling with an inert gas is necessary because the laser power at the exit of the TSF is sufficiently high to ionize the gases in air causing stimulated rotational Raman scattering (SRRS). The BTS GCS is responsible to maintain the beam tubes with a carefully controlled concentration of argon gas at a specific temperature and humidity. 
Integrated Computer Controls System Description

\subsubsection{Safety Interlock System}

The Safety Interlock System (SIS) provides personnel safety interlocks for the entire facility. It continuously displays the hazard level in the facility and annunciates changes in hazard level which may include Laser Light, High Voltage, Ionizing Radiation, and Oxygen depletion. The SIS monitors door, hatch, and shutter positions, crash buttons, oxygen sensors, and radiation levels. It provides hardware permissive signals to power conditioning, safety shutters, and other components as necessary to protect personnel, and warn them of the hazards in the area. It provides a digitized voice annunciation of hazard level changes in the facility.

\subsubsection{Access Control System}

The Access Control System (ACS) is used to control access into and within areas of the facility. It operates in conjunction with the SIS using an on-line database of currently qualified personnel. The ACS tracks entry and egress through monitored doors by sensing special badges carried by all personnel in the facility. All movement into or out of the bays is recorded in a permanent transaction log.

\subsection{6 t-1 Abort System}

The $\mathrm{t}-1$ Abort System monitors key components in the Input Sensor, Output Sensor, and Cavity Spatial Filters of each beam with the responsibility to initiate an abort of the system shot during the final one second if these components do not reach their proper shot configuration. Device controls are the responsibility of the Alignment Controls FEP. The abort function is implemented by the Integrated Timing System.

\subsubsection{Facility Environmental Monitor}

The Facility Environmental Monitor (FEM) monitors environmental parameters throughout the NIF that affect laser performance and general operations. These parameters include temperature, relative humidity, oxygen content, pressure, vacuum, moisture, and $\mathrm{pH}$.

\subsubsection{Target Chamber Vacuum System}

The Target Chamber Vacuum Control System (TCVCS) evacuates and maintains a vacuum of $10^{-5}$ Torr in the Target Chamber. This system is a multiple stage vacuum system with roughing, turbo, and cryo pumps. A variety of instruments are used to measure the vacuum pressure of the range from atmosphere to high vacuum.

\subsubsection{Environmental Protection System}

The Environmental Protection System (EPS) consists of the Neutron and Gamma Monitoring System, the Tritium Monitoring System, and the EPS Exhaust System. This system monitors and displays:

- Neutron levels in the control room, and the diagnostic control room, 
Integrated Computer Controls System Description

- Gamma levels in the control room, diagnostic control room, target bay access ways, and the Elevated Release Point (ERP),

- Tritium levels in the target bay, hoods, glove-boxes, target storage area, and the ERP.

\subsubsection{Tritium Processing System}

Two Tritium Processing Systems (TPS) operate with the target chamber vacuum system to recover tritium that may be released into the NIF target chamber due to a breached target, power failure, pump failure, or other abnormal event. The TPS also processes gas released from the cryo pumps during regeneration.

\subsubsection{Personnel, Safety, and Occupational Access}

The Personnel, Safety, and Occupational Access System provides safe transport pathways into and out of the target area for the occupational access of personnel and equipment to the different systems in the target area. This system monitors oxygen and carbon dioxide levels within the hazardous areas of the diagnostic building, and the atmosphere inside the target chamber during manned-access operations.

\subsubsection{Final Optics Assembly Thermal Control System}

The Final Optics Assembly (FOA) Thermal Control System (TCS) performs closedloop temperature control of the FOAs. The TCS monitors the temperature and flow status of each of the 48 FOAs, the temperature of each of the $192 \mathrm{KDP}$ crystals, and controls the recirculation pumps, heat exchangers, and water heaters.

\subsection{Alignment FEP}

The alignment control FEP [Figure 28] integrates 9000 motorized and 3500 binary actuators. Typical optical-mechanical devices being controlled include mirrors, actuators, pinhole wheels, waveplates, etc. These devices vary in complexity from simple dual-state control to sophisticated cross-coupled motion of several components.

There are 6 major geographical subdivisions of the total beam alignment system:

- Pre-Amplifier Beam Transport System (PABTS), and Input Sensor

- Cavity Spatial Filter / Main beam line

- Output Sensor Package / Transport Spatial Filter

- Precision Diagnostics / Roving Mirror

- Turning Mirrors / Final Optics Assembly

- Target Alignment

Motion control commands available for remote control of alignment devices include 1) goto relative or absolute position, 2) goto limit, and 3) goto predefined setpoint. The software parameterizes velocity profiles, accelerations, backlash correction, and independent or synchronized motion between multiple axes. Upon request, the 
status of devices is sent to client software. Device operating data is logged for trending purposes.

The design employs one set of embedded controllers for motors and another set for binary devices. Oregon Micro Systems (OMS) VME58 family of VME based embedded motor controllers provides the stepper motor control functions. The VME58 family also provides servomotor control, which is slated for a few applications in the NIF. The VME58 can control 8 axes per board. One rack contains a single FEP that is capable of controlling up to 128 motors.

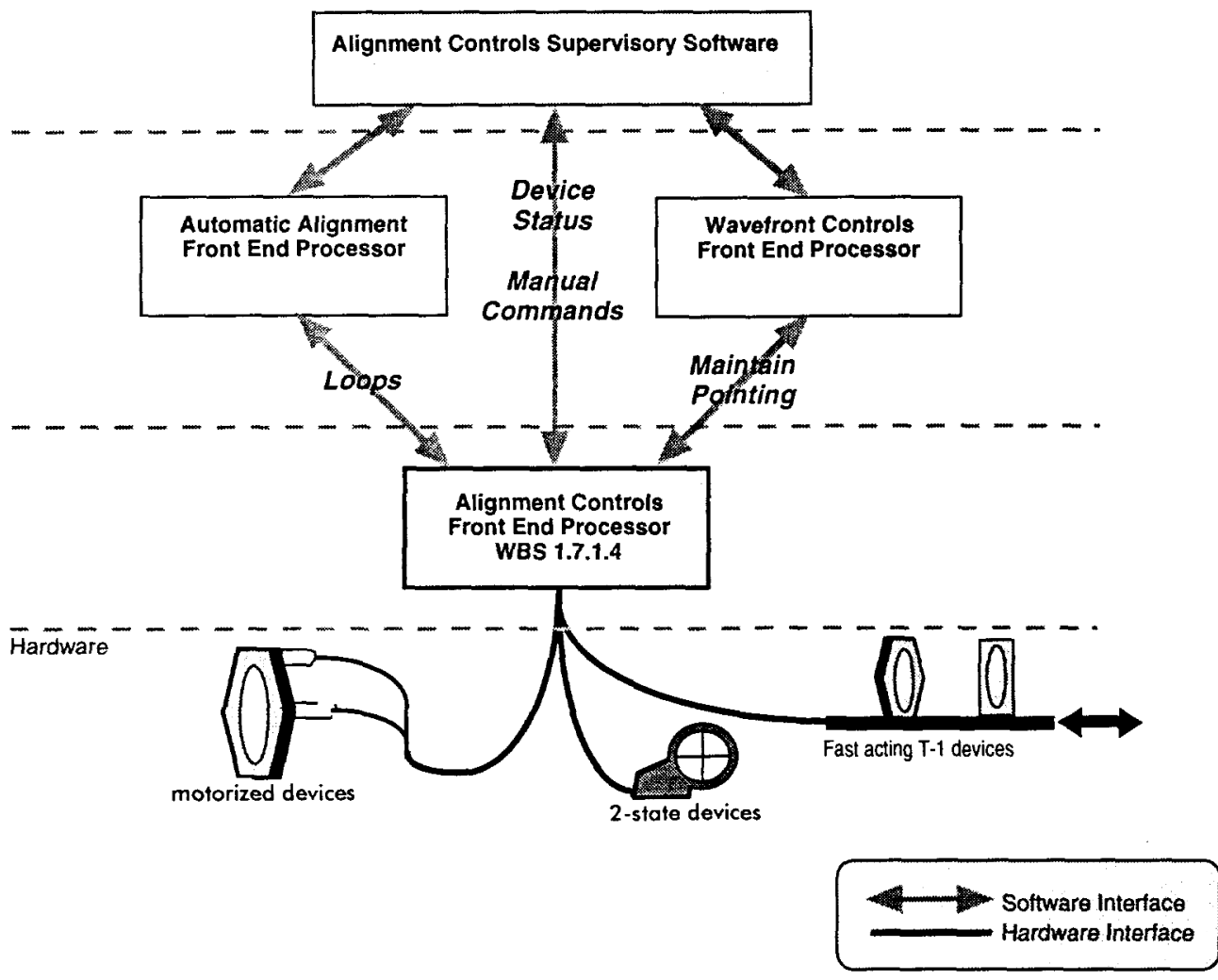

\section{Figure 28 Alignment control FEP architecture}

Some alignment devices have special requirements. For example:

- Wheels are used in the Cavity Spatial Filter and the Transport Spatial Filter to carry a number of pinholes. Pinholes must be placed accurately so that the beams are not clipped. A position verification switch was added to verify location a "home" position. Then by counting the number of steps of motion from the home position, the actual position of the wheel is verified. 
- "T-1 Abort System Devices" consist of 4 devices per beamline (approximately 800 total) that must be moved within the last second before the laser shot. (These devices are an integral part of the wavefront control system). It sets most alignment devices to their shot positions ahead of time, receives an arm command from the Alignment Supervisory System, and waits to be triggered by the Integrated Timing System. Once triggered, an interrupt service routine is executed that quickly issues the motion commands.

\subsection{CCD Camera FEP}

Charged Coupled Device (CCD) imaging sensors are employed in a variety of situations to help diagnose the laser beams. This FEP provides a common interface for all camera types to reduce software development and maintenance costs.

There are 5 variations of the CCD Camera FEP [Figure 29]:

- Streak Cameras for the Roving Diagnostics

- Optics Inspection

- Scientific grade (i.e. high resolution and/or high dynamic range) CCD cameras

- Streak cameras for $1 \omega$ diagnostics

- Streak cameras for $3 \omega$ diagnostics

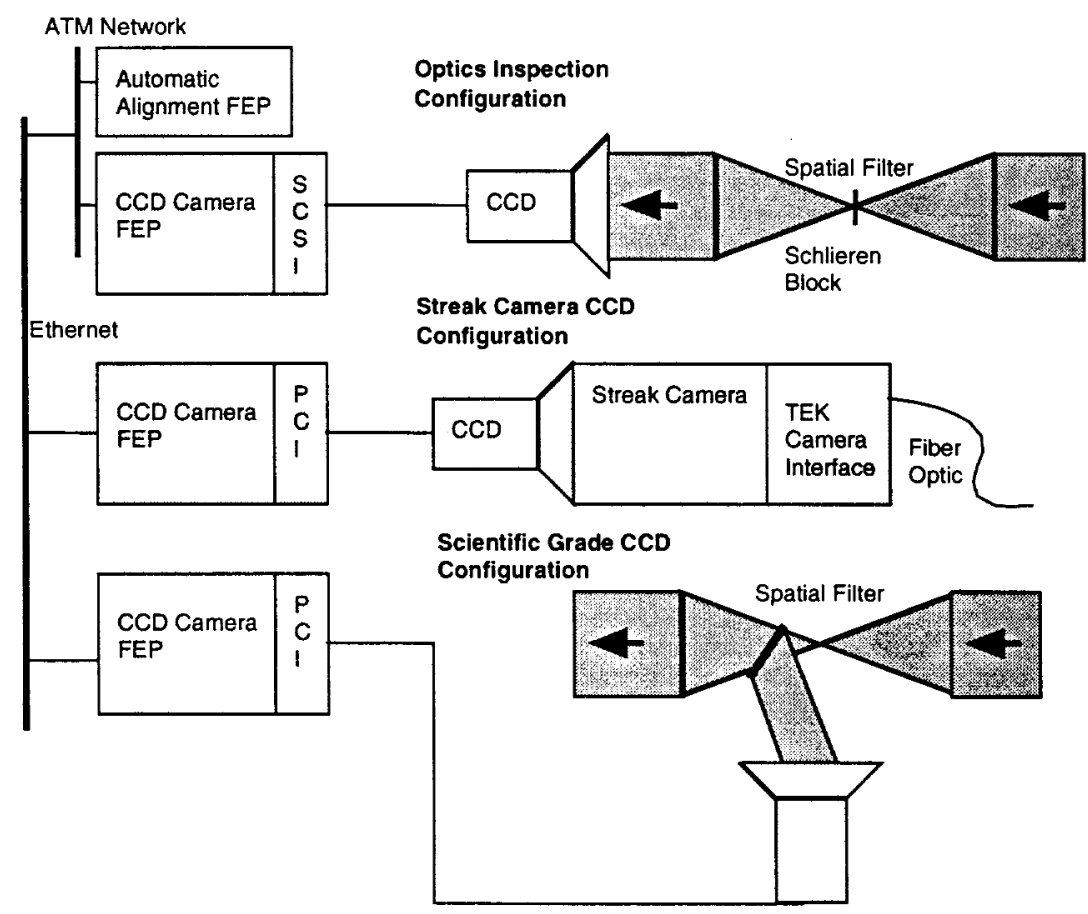

Figure 29 CCD camera controller FEP architecture 


\subsubsection{Optics Inspection CCD}

CCD cameras are used for computerized optics inspection in the beamline. Damage to optics due to high-power laser shots progressively degrades performance to the point of potential total failure. Optics failure is especially catastrophic in the Cavity and Transport Spatial Filters and the Final Optics Assembly since these optics are also vacuum barriers. The goal of the Optics Inspection System is to detect and monitor optical flaws in the components and notify personnel when optical damage sites exceed limits.

\subsubsection{Streak Cameras}

Streak cameras record very high-speed events with both the temporal shape and arrival time of laser pulses. CCD cameras digitize the streak camera output to be analyzed to reconstruct the event being observed. Several systems are used in NIF. The Roving Streak Camera is on a roll around cart for portable diagnostic use. Both $1 \omega$ and $3 \omega$ laser light is diagnosed. The Pulse Synchronization Streak Camera examines the arrival times of the laser onto the target.

\subsubsection{Scientific Grade CCD}

CCD cameras also examine near-field and far-field images of the laser beams in order to observe the flatness of the laser beam, fill factor, fringe patterns, and other phenomena that might occur while the beam propagates toward the target.

\subsection{Laser Energy Diagnostics FEP}

The laser energy diagnostics FEP [Figure 30] uses silicon photodiodes and calorimeters to monitor laser energy at key points along the beamline. These measurements are used to tune laser power balance and record laser performance. The system consists of six VME-based FEP located in the laser bays and switchyards. Detectors are remotely located from the FEP using LONworks embedded controllers. Measurements are made at the Input Sensor for $1 \omega$ input energy, the Output Sensor for $1 \omega$ output energy, and the Final Optics Assembly for $3 \omega$ output energy. Calorimeters are used at the Input Sensor and switchyard wall Roving Calorimeter for on-line calibration of the photodiodes. Support of the Precision Diagnostics calorimeters and photodiodes are also included. 


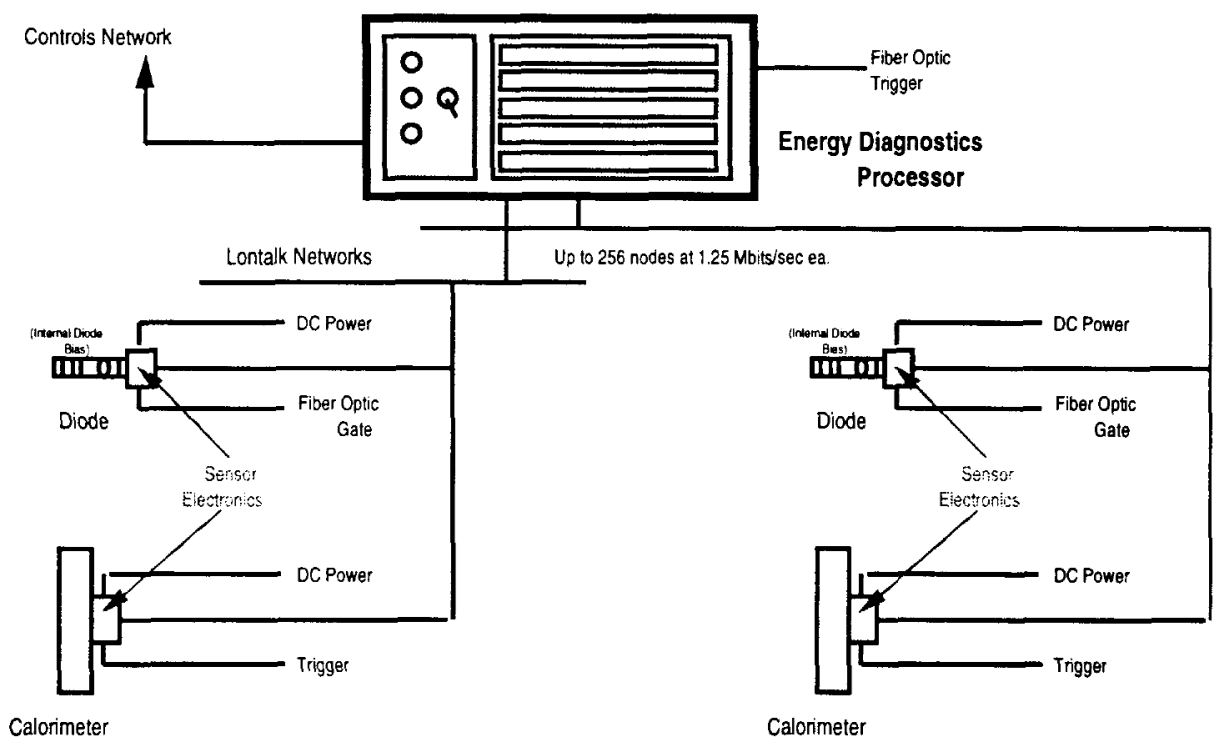

Figure 30 Laser energy diagnostic FEP architecture

For proper operation, the energy sensors must be set to acquire in the optimum range at the sensor locations. In addition, the system verifies and sets the trigger delay and integration gate width for the photodiodes to time and frame the pulse correctly. Calorimeters calibrate the photodiode sensors. A self-test of the sensors validates proper operation.

Firmware in the sensor embedded controller performs early processing of the data. Upon command from the FEP, the photodiode controller starts by sequencing an analog-to-digital converter (ADC) to collect a background reading for data correction and applies that correction to the measurement before transmitting the data to the FEP. The calorimeter controller records both pre- and post-trigger data. The data is curve-fit to an exponential function to determine the energy.

\subsection{Laser Power Diagnostic FEP}

The Power Diagnostics FEP [Figure 31] measures the non-calibrated temporal shape of the laser beam. Using this information and the energy measured at the same point the laser beam power is derived. The system consists of four rack-mountable SUN workstations, each controlling multiple four-channel transient digitizers housed in three VXI crates. The crates are interfaced to the SUN workstation with a PCI-bus to MXI-bus adapter and a slot-0 MXI/VXI controller. 


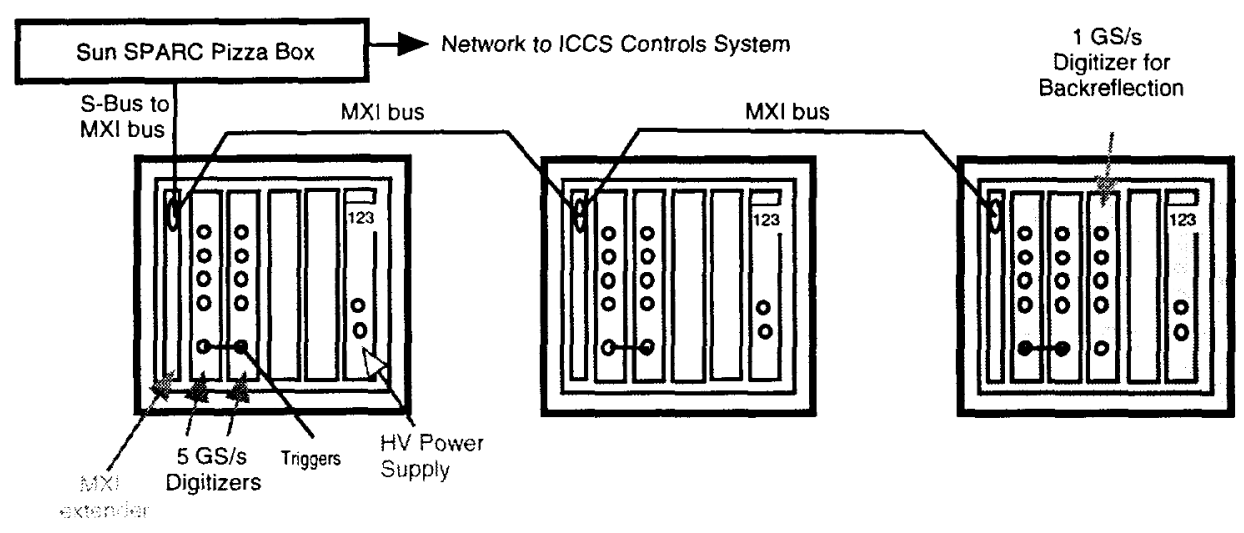

Figure 31 Laser power diagnostics FEP architecture

The signals input to the digitizers originate as optical waveforms from the sensor packages that are time-multiplexed via fiber optics onto vacuum photodiodes. This time multiplexing allows multiple beams waveforms to be recorded onto one digitizer channel. The vacuum photodiode converts the optical waveforms into electronic signals, divides them into four separate signals to increase dynamic range by inputting them into the four separate digitizer channels using different gain and offset settings. These measurements are made at the Input Sensor for $1 \omega$ input power and at the Output Sensor for $1 \omega$ and $3 \omega$ output power. The vacuum photodiode requires a high voltage bias that is controlled by an Ethernet to GPIB converter attached to a programmable power supply. In addition, a lower bandwidth transient recorder is used to measure laser back-reflection on a few beams.

Data are reconstructed by collecting four separate records from different digitizer channels. The records are temporally aligned using a recorded fiducial signal for registration, and then scaling and offsetting each portion to construct a single waveform.

\subsection{Wavefront FEP}

The wavefront FEP [Figure 32] controls adaptive optics that are required to improve the beam quality so that the laser meets precise propagation, target alignment, and target focusing specifications. During laser alignment, a control loop corrects for thermally induced aberrations and optical errors (mounting, polishing and other low spatial frequency defects). During the shot, the system provides feed-forward correction in anticipation of pump-induced and whole-beam self-focusing errors.

The wavefront is measured by a Hartmann sensor within the Output Sensor using a sample of the beam taken from the Transport Spatial Filter (TSF). The deformable mirror (located at the backside of the large four-pass amplifier) can actively correct wavefront errors up to the TSF and anticipate necessary corrections beyond to the Final Optics Assembly. 


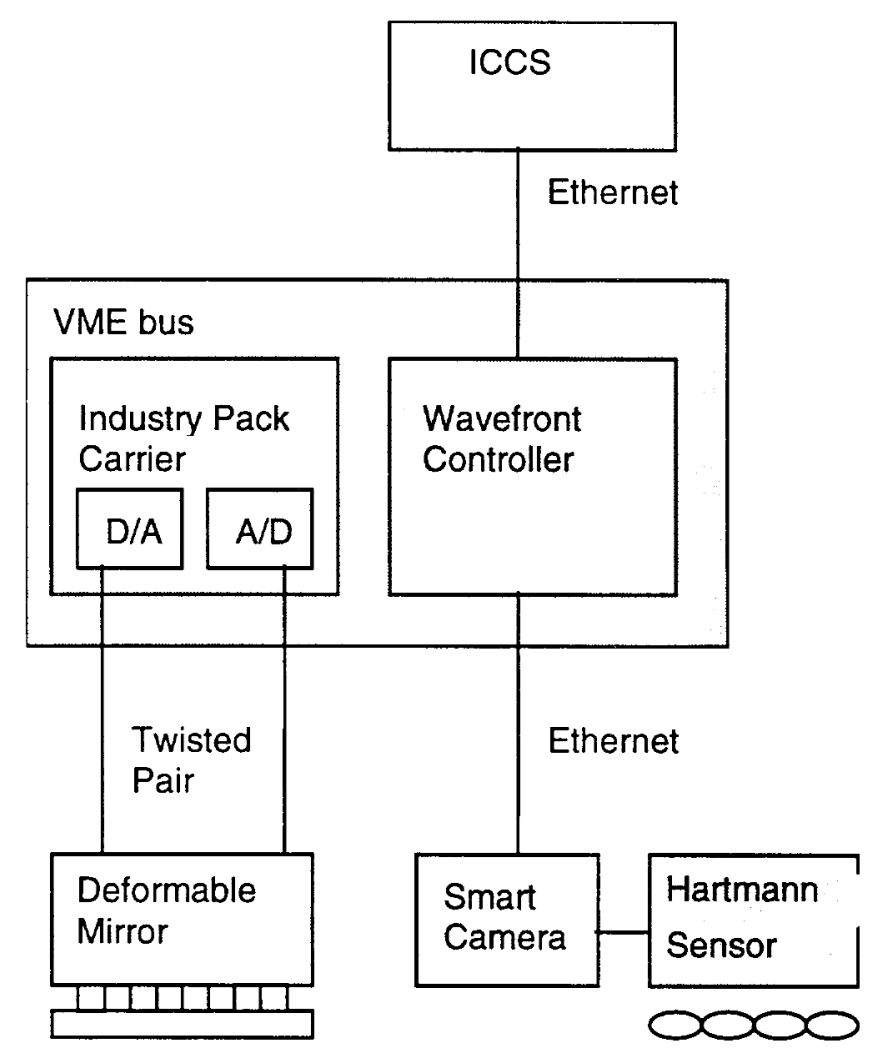

Figure 32 Wavefront Control System FEP architecture

There are two key control points in each beam for wavefront correction - a deformable mirror, and a wavefront sensor. The surface of the mirror is deformed to correct wavefront errors by controlling 39 actuators organized in small zones. The Hartmann sensor contains 77 small lenslets, which in effect sample the average wavefront (as a pointing error) in the small areas. The centroid of each lenslet's spot is measured on a CCD camera by the FEP, which computes the overall corrections necessary and deflects the mirror actuators to correct the wavefront. The Hartmann sensor interfaces directly to a self-contained camera containing a digital image processor.

Closed loop corrections continue to within 1 second of the laser shot. Shortly before the shot, open loop correction of anticipated errors (as described above) is performed. After the shot, the performance of wavefront correction is recorded. The centroid data passes through Ethernet to the FEP. Each wavefront control processor controls four deformable mirrors in closed loop at a $10 \mathrm{~Hz}$-processing rate. 


\subsection{Target Diagnostics FEP}

The target diagnostics FEP [Figure 33] controls a variety of diagnostics to diagnose target performance. Initially, the FEP will be capable of controlling the Diagnostic Instrument Manipulator (DIM), the Time Resolved X-Ray Imaging System (TRXI), the X-Ray Streak Slit Camera (XSSC), and the Static X-Ray Imaging System (SXI). These diagnostics will be distributed throughout the target area. Additional diagnostics will be added as the facility matures. A diagnostic controller integrates each diagnostic into the FEP. The diagnostic controller is a computer of unspecified make and model that is furnished by the experimenter.

The target diagnostics FEP controls a variety of experimental diagnostics that will initially be used to diagnose laser system performance and verification tests on target. Afterwards the target diagnostic FEP will control all of the diagnostics associated with ignition, weapons physics, and other experimental programs. Initially, the FEP will be capable of controlling the Diagnostic Instrument Manipulator (DIM), the Time Resolved X-Ray Imaging System (TRXI), the X-Ray Streak Slit Camera (XSSC), and the Static X-Ray Imaging System (SXI).

Figure 33 shows a simplified, one-line depiction of the target area data acquisition system. The diagnostic FEP provides the interface between the supervisory ICCS and the various diagnostic experimental systems. Each diagnostic will have its own controller that knows all about the experimental equipment that composes that particular diagnostic, what type of data is to be collected, how the equipment is to be set up and controlled, and how to communicate with the FEP. 


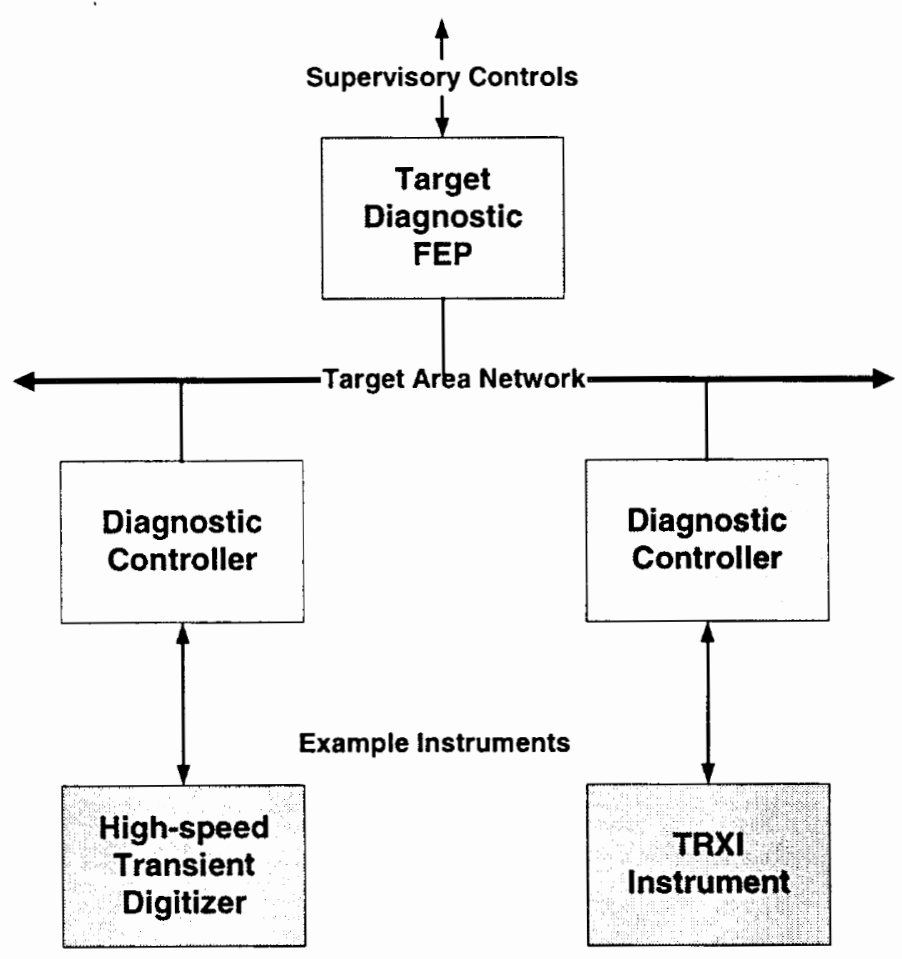

Figure 33 Target diagnostics FEP architecture

The diagnostic controller is used for initial diagnostic checkout, stand-alone operation, and general operational tasks in conjunction with the FEP. Since the various diagnostics are presently planned to be developed by a variety of different organizations, the diagnostic FEP provides a common hardware and software interface to the supervisory system.

The FEP receives general commands from the ICCS, interprets the commands and passes the commands to the appropriate diagnostic controllers. The FEP collects status and data from the various diagnostics, and formats the data before sending it back to the supervisory system for archiving.

The target area data acquisition system is required to process both unclassified and classified data. Design constraints are placed on the target area data acquisition system (which includes the FEP) to satisfy the requirements of classified operation.

Classified data processing constraints include:

- Placing all cabling in locked trays,

- Using only removable disk drives for all computers,

- Providing for inspection of all components not contained in locked enclosures, and

- Approved operational procedures for changing the system from unclassified to classified operation and back. 


\subsection{Prototype Alignment and Automatic Alignment FEPs}

The Alignment Concepts Lab contains a simulated portion of the main laser segment of the NIF beamline [Figure 34]. Each of the labeled items are controlled by using 16 stepping motors, ( 6 mirror gimbals and 4 reference inserters), and 4 binary controls ( 3 shutters and a halogen lamp).

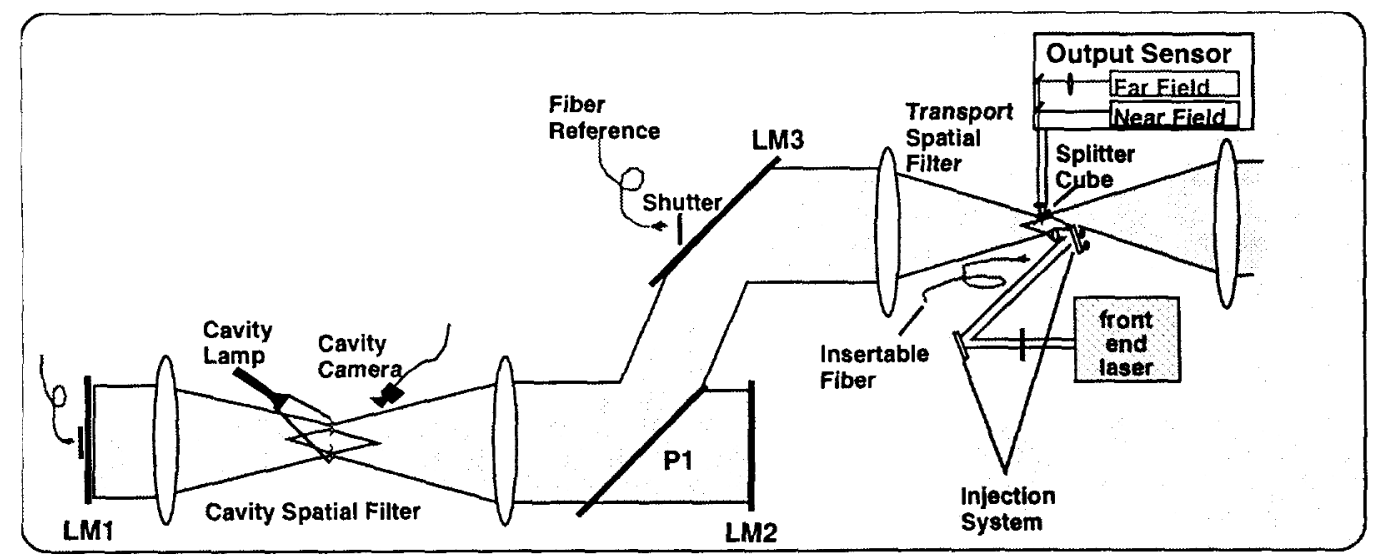

Figure 34 Alignment Concepts Lab beamline configuration

The combination of the prototype Automatic Alignment FEP and the Alignment FEP perform control of the simulated beamline in the following loops:

- Center LM1 to LM3

- Center injected beam to LM3

- Point through pass 1 of TSF

- Center injected beam to LM3

- Point through pass 1 of TSF (repeated)

- Point through pass 3 of CSF

- Point through pass 4 of CSF

- Point through pass 4 of TSF

- Center LM1 to LM3

Functions performed to accomplish this control include 8-axis (motor) cross-coupled motion correction to align the four cavity optics (LM1, LM2, LM3, P1). Persistent storage, using non-volatile memory, is provided to retain position and setpoint information through hardware and software shutdowns and restarts.

The prototype FEPs have been used to demonstrate completely automatic alignment of the simulated beamline, in many cases meeting or exceeding the NIF tolerances for the corresponding alignment loops. 
Also, emulation capability was constructed that demonstrates the parallel alignment of the $96 \mathrm{NIF}$ beamlines by a single computer process and confirms the predicted alignment time.

The relationship between the prototype Automatic Alignment FEP, the prototype Alignment FEP, and various electronic and electro-mechanical devices with the laser that simulates a portion of the NIF beamline is shown in Figure 35.

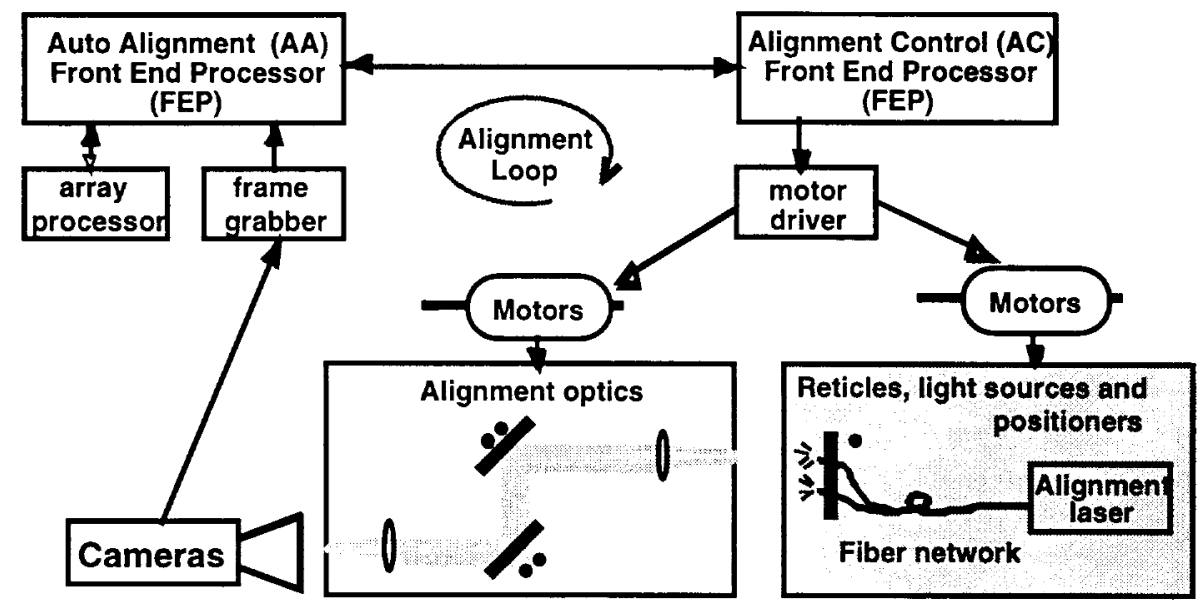

Figure 35 Alignment prototype FEP architecture

Information is loaded from the Configuration database Framework allowing the Alignment FEP to dynamically determine (at run-time) the set of devices it controls, their static parameters, and their possible association or grouping with other devices. Static parameters include acceleration and maximum velocity of motors. Device associations are used for cross-coupled operations between motors. Further information may be found in reference [9]. 
Integrated Computer Controls System Description

This page intentionally left blank. 


\section{Summary}

The ICCS is being developed using the iterative approach to software construction [10]. This technique is proven effective for projects whose requirements are not fully known until late in the project development. Five iterations are planned prior to the first facility deployment of the ICCS software. Each new release will follow a plan aimed at addressing the greatest risks to the architecture.

At this early stage in the ICCS design, the first prototype release has been completed and demonstrated on a computer testbed [Figure 36]. The testbed includes several FEPs, a small tabletop alignment system, and a network of switched Fast Ethernet and ATM. It currently demonstrates integrated operation of six of the frameworks collaborating over CORBA communications.

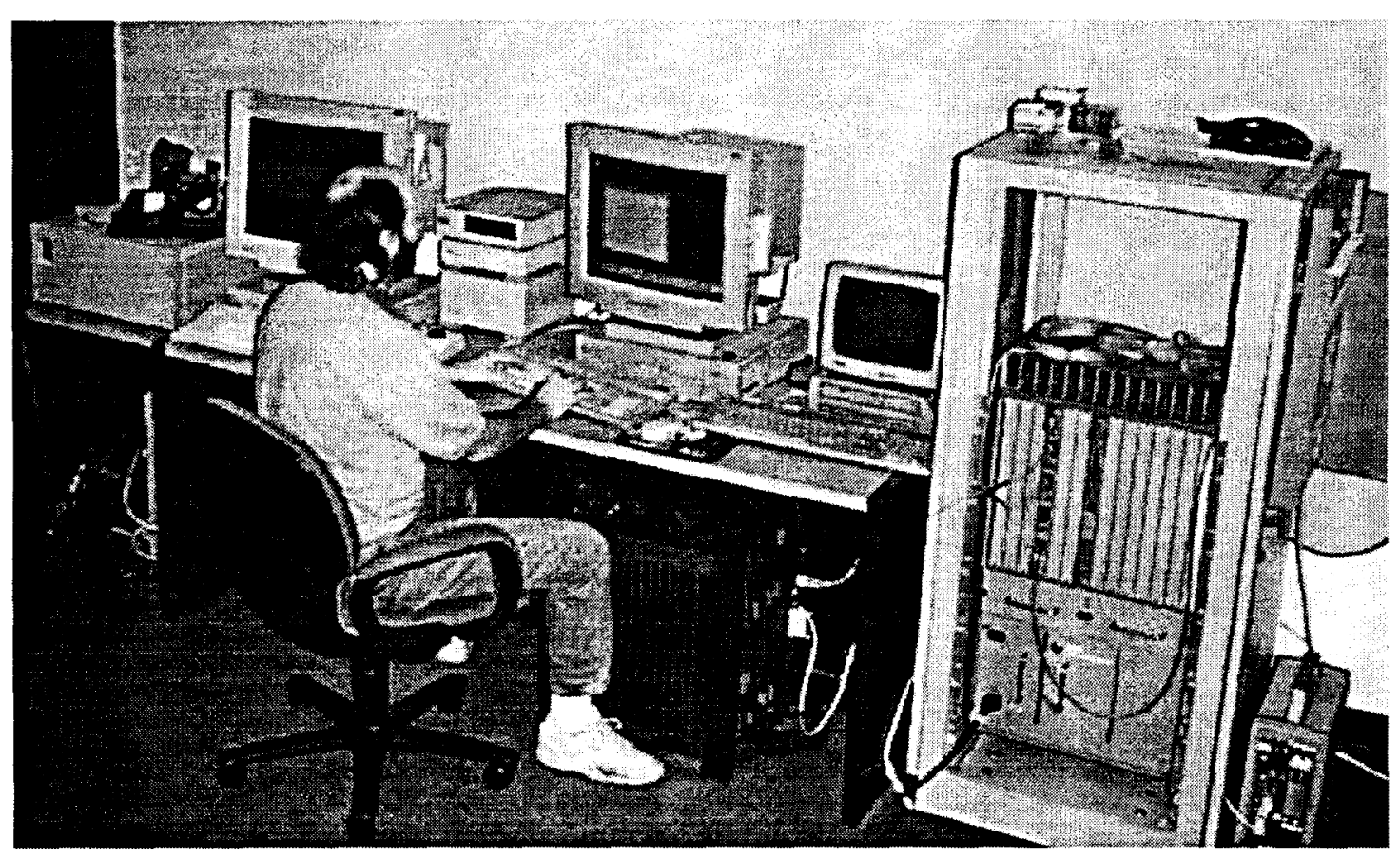

Figure 36 Integrated computer control system testbed

A second iteration on the prototype is now complete [11]. Enhancements were directed at four goals:

1. Deploy the framework architecture onto additional FEPs and develop a tutorial document to specify the process that engineers use to construct specific products. 
2. Increase the degree of distribution and functionality of the Status Monitor and Configuration frameworks.

3. Introduce database management into device configuration and FEP initialization.

4. Improve the fault resistance of the frameworks

Following these early demonstrations, the collection of the next three iterations have been termed the Production Prototype. The first phase, which occurs in the summer of 1998, will deliver vertical slices of all applications necessary for the NIF control system. Subsequent phases will be constructed during 1999 leading toward first deployment in the facility in the year 2000, when the first 8 of the 192 beams will be operated.

Construction of the ICCS incorporates many of the latest advances in computer and software technology. Primary goals of the design are to provide an open, extensible, and reliable architecture that can be maintained and upgraded for decades. The design permits software reuse and allows the system to be constructed within budget. As an added benefit, the design is sufficiently abstract to allow future control systems to take advantage of this work. 
Integrated Computer Controls System Description

\section{Appendix - Acronyms}

ACS

API

ATM

AWG

BTS

CCD

CORBA

CPU

CSF

DBMS

EPS

ERP

FEM

FOA

FEP

FET

GCS

GPIB

GUI

HDF

ICS

ICCS

IDL

ISS

LM

LON

LRU

MOR
Access control system

Application programming interface

Asynchronous transfer mode

Arbitrary waveform generator

Beam transport system

Charge-coupled device

Common object request broker architecture

Central processing unit

Cavity spatial filter

Database management system

Environmental protection system

Elevated release point

Facility environmental monitor

Final optics assembly

Front-end processor.

Field-effect rransistor

Gas control system

General purpose instrumentation bus

Graphical user interface.

Hierarchical data file

Industrial controls system

Integrated computer control system.

Interface definition language

Integrated safety system

Laser mirror

Local operating network

Line replaceable unit

Master oscillator room 
Integrated Computer Controls System Description

MPA

MXI

OPG

PABTS

PAM

PCI

PCU

PDM

PEPC

PILC

PLC

RAM

SCL

SBS

SDD

SQL

SSD

SRS

Tcl/Tk

TCP/IP

TCS

TPS

TSF

VCS

VME

VXI
Multipass amplifier

Multi-system extension interface

Optical pulse generation

Preamplifier beam transport

Preamplifier module

Peripheral connection interface

Power conditioning unit

Product data management

Plasma electrode Pockels cell

Pulsed ionization lamp check

Programmable logic controller

Reliability, availability, maintainability

Sequence control language

Stimulated Broulloin scattering

Software design description

Structured query language

Smoothing by spectral dispersion

Software requirement specification

Tool command language / toolkit

Transport control protocol / Internet protocol

Thermal control system

Tritium processing system

Transport spatial filter

Vacuum control system

Versa module Eurocard

VME extension interface 


\section{References}

1. J. A. Paisner and J. R. Murray, "The National Ignition Facility for Inertial Confinement Fusion", UCRL-JC-128689

2. "ICCS Glossary of Terms", NIF-760

3. "NIF Shot Life Cycle: ICCS Supervisory Software Responsibilities", NIF-96-987

4. "Engineering Analysis and Literature Review of the use of CORBA in Distributed Object-Oriented Systems", NIF-314 (revised June 1997)

5. "Software Quality Assurance Plan for the National Ignition Facility Integrated Computer Control System", NIF-288

6. G. Booch, "Object-Oriented Analysis and Design", Benjamin/Cummings Publishing Company, Inc., Second Edition 1994

7. V.Swaminathan and J. Storey, "Domain-Specific Frameworks", Object Magazine, April 1988, pp. 53-57

8. "ICCS Device Naming Convention", NIF-2435

9. B. Boehm, "A Spiral Model of Software Development and Enhancement", IEEE Computer, May 1988, pp. 61-72

10. E. Stout, "CS \& T Alignment Controls Prototype FY97 Year-End Report", October 1, 1997.

11. J. Woodruff, "FY97 ICCS Prototype Final Report", January 5, 1998, NIF-4307 